\title{
The roles and mechanism of cocatalysts in photocatalytic water splitting to produce hydrogen
}

\author{
Nan Xiao ${ }^{\text {a,b }}$, Songsong Li ${ }^{b}$, Xuli Li ${ }^{b}$, Lei Ge a,b,*, Yangqin Gao b, Ning Li b \\ a State Key Laboratory of Heavy Oil Processing, College of New Energy and Materials, China University of Petroleum Beijing, Beijing 102249, China \\ b Department of Materials Science and Engineering, College of New Energy and Materials, China University of Petroleum Beijing, Beijing 102249, China
}

\section{A R T I C L E I N F O}

\section{Article history:}

Received 26 September 2019

Accepted 17 October 2019

Published 5 April 2020

\section{Keywords:}

Cocatalysts

Photocatalytsts

Hydrogen evolution

Charge separation

Water splitting

\begin{abstract}
A B S T R A C T
Photocatalytic hydrogen $\left(\mathrm{H}_{2}\right)$ evolution via water spilling over semiconductors has been considered to be one of the most promising strategies for sustainable energy supply in the future to provide non-pollution and renewable energy. The key to efficient conversion of solar-chemical energy is the design of an efficient structure for high charge separation and transportation. Therefore, cocatalysts are necessary in boosting photocatalytic $\mathrm{H}_{2}$ evolution. To date, semiconductor photocatalysts have been modified by various cocatalysts due to the extended light harvest, enhanced charge carrier separation efficiency and improved stability. This review focuses on recent developments of cocatalysts in photocatalytic $\mathrm{H}_{2}$ evolution, the roles and mechanism of the cocatalysts are discussed in detail. The cocatalysts can be divided into the following categories: metal/alloy cocatalysts, metal phosphides cocatalysts, metal oxide/hydroxide cocatalysts, carbon-based cocatalysts, dual cocatalysts, Z-scheme cocatalysts and MOFs cocatalysts. The future research and forecast for photocatalytic hydrogen generation are also suggested.
\end{abstract}

(C) 2020, Dalian Institute of Chemical Physics, Chinese Academy of Sciences. Published by Elsevier B.V. All rights reserved.

\section{Introduction}

Due to the world's heavy reliance on non-renewable, geopolitically sensitive energy sources such as natural gas and coal, coupled with the violent fluctuations in crude oil prices, ensuring alternative clean and renewable energy supplies has never been such urgent [1-6]. The world's primary energy consumption increased by $2.9 \%$ in 2018 , which is the highest annual growth rate since 2010, almost double of the average annual growth rate in the last 10 years. Our main energy sources still depend on limited and non-renewable fossil fuels, such as oil, natural gas and coal. Besides, the combustion of fossil fuels has led to a series of serious environmental pollution issues, such as air pollution, water pollution and even global warming.
Therefore, it is imperative to develop renewable, clean and environmentally-friendly energy sources. Solar energy is the most tremendous renewable natural resource, which is abundant, cheap and clean [7-11]. $\mathrm{H}_{2}$ is considered as an important energy in the future, which is mainly due to its cleanness, non-toxicity and high energy density compared to other natural resources. Photocatalytic hydrogen production via solar splitting is one of the most promising solutions to address the environmental problems and sustainable clean energy issues.

Since the pioneer work of Fujishima et al. [12] on photoelectrochemical water splitting to produce hydrogen $\left(\mathrm{H}_{2}\right)$ and oxygen $\left.\mathrm{CO}_{2}\right)$ separately under light irradiation by using $\mathrm{TiO}_{2}$ in 1972 , semiconductor photocatalytic water splitting to produce $\mathrm{H}_{2}$ as a potential technology has received extensive attention

\footnotetext{
* Corresponding author. E-mail: gelei08@sina.com

This work was financially supported by the National Natural Science Foundation of China (51572295, 21273285 and 21003157), Beijing Nova Program (2008B76), and Science Foundation of China University of Petroleum, Beijing (KYJJ2012-06-20 and 2462016YXBS05). DOI: S1872-2067(19)63469-8 | http://www.sciencedirect.com/science/journal/18722067 | Chin. J. Catal., Vol. 41, No. 4, April 2020
} 
and rapid development [11,13-18]. In the past two decades, the semiconductor photocatalysts have been greatly developed by a large number of scholars from various countries and regions. Many photocatalytic materials have been investigated, including metal oxides or sulfides, such as $\mathrm{TiO}_{2}$ [19-28], CdS [29-38] and ZnCdS [15,39-42], and the metal-free semiconductors such as $\mathrm{rGO}$ [43] and $\mathrm{g}-\mathrm{C}_{3} \mathrm{~N}_{4}$ [5,44-54]. However, the photocatalytic activities of the most promising semiconductor photocatalysts are still not quite satisfied due to the easy recombination between charge carriers. In order to suppress the charge recombination, many strategies have been developed for effective separation of electron-hole pairs, including surface loading of cocatalysts [55-57], turning the morphologies $[48,58,59]$, heterojunctions/homojunctions [60-62], molecular design [63-67] and heteroatom doping [5,68]. Cocatalyst loading is an effective strategy to promote the photocatalytic activity of semiconductor photocatalysts, in which way the stability of the photocatalysts, the separation efficiency of the photogenerated charges, the number of exposed active sites and the light harvesting ability could be largely enhanced. Therefore, cocatalysts play an important role in improving the performance of photocatalytic $\mathrm{H}_{2}$ evolution. Until now, a large number of strategies were developed to modify semiconductor photocatalysts by cocatalysts, such as Type I heterojunction $[69,70]$, Type II heterojunction [61], p-n heterojunction $[62,71,72]$, Schottky-junction $[73,74]$, Z-scheme heterojunction [75-78] and S-scheme heterojunction [14], which have been widely designed to form build-in electric field for boosting the charge carrier separation, thus achieving improved photocatalytic hydrogen production.

In this review, we focus on the roles and mechanisms of cocatalysts developed to date for photocatalytic $\mathrm{H}_{2}$ evolution, including (1) metals, alloys, metal phosphides, metal oxides, metal hydroxides, metal sulfides, carbon-based, etc., as single cocatalysts for $\mathrm{H}_{2}$ evolution, (2) the synergetic effect of the dual cocatalysts loading for reduction reaction, (3) the effect of Z-scheme for water splitting, and (4) the improvement of $\mathrm{H}_{2}$ production by loading MOFs as cocatalysts. We pay attention to the roles and mechanisms of various cocatalyst systems with important and representative research being discussed and summarized.

\section{Fundamentals of photocatalysis and roles of cocatalysts}

\subsection{Fundamentals of photocatalysis}

In general, the reaction mechanism of photocatalytic water splitting for $\mathrm{H}_{2}$ production involves three major steps [79]: (1) absorption of light by semiconductor photocatalysts to generate electron $\left(e^{-}\right)$-hole $\left(h^{+}\right)$pairs; (2) charge separation and migration to the surface of semiconductor photocatalysts; and (3) surface reactions for water reduction and oxidation, respectively. The efficiency of photocatalytic $\mathrm{H}_{2}$ evolution is determined by the kinetics of the above three steps altogether (Eq. 1):

$$
\eta=\eta_{1} \times \eta_{2} \times \eta_{3}
$$

where $\eta_{1}$ is the efficiency of light absorption, $\eta_{2}$ is the efficiency of charge separation, and $\eta_{3}$ is the efficiency of surface catalytic reaction.

Generally, the semiconductor photocatalyst is composed of vacant conduction band (CB) and filled valence band (VB), which are separated from each other by $E_{\mathrm{g}}$. The photocatalytic process begins with light absorption, when the photon energy is larger than or equal to the band gap $\left(E_{\mathrm{g}}\right)$, photon absorption in the photocatalyst causes electronic transition and generates $e^{-}-h^{+}$pairs, then the electrons in the VB are excited to the CB, while the holes are left in the VB. The electrons in the $\mathrm{CB}$ participate in the reduction reaction to produce $\mathrm{H}_{2}$, and the oxidation reaction is driven by the holes in the VB. The minimum band edge of the $\mathrm{CB}$ has to be more negative than the redox potential of $\mathrm{H}^{+} / \mathrm{H}_{2}(0 \mathrm{~V} v s$. RHE), while the maximum band edge of the $\mathrm{VB}$ should be more positive than the chemical redox potential of $\mathrm{O}_{2} / \mathrm{H}_{2} \mathrm{O}$ (Fig. 1). However, if there are no suitable activity sites or reaction center on the surface of the semiconductor photocatalyst, the photogenerated electrons and holes could quickly recombine in the bulk of the semiconductor. According to the previous reports $[9,80,81]$ that the recombination rate of photoinduced electrons and holes in the bulk of the catalysts is very fast (ps to ms). Therefore, the electrons and holes should be quickly captured by the cocatalysts for efficient separation and transfer of photogenerated charges and participate in the reduction reactions on the surface, which is critically important for photocatalytic water splitting.

\subsection{Roles of cocatalysts in photocatalytic $\mathrm{H}_{2}$ evolution system}

In photocatalytic $\mathrm{H}_{2}$ evolution system, the cocatalysts have four pivotal roles in enhancing the activity of the semiconductor photocatalysts (Fig. 2): (1) Cocatalysts can enhance the light
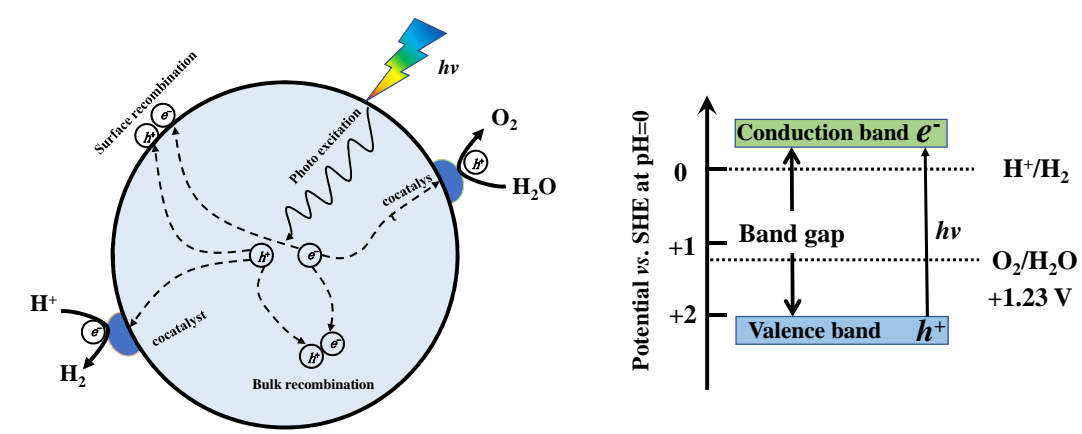

Fig. 1. The mechanism of photocatalytic water splitting in a semiconductor photocatalyst system. 


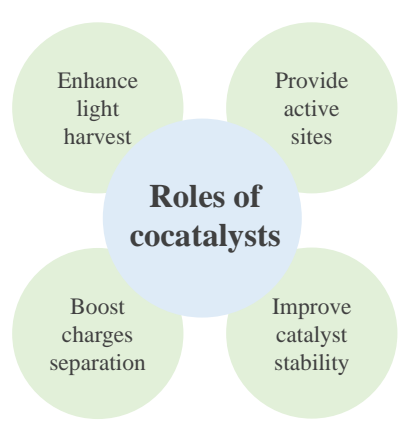

Fig. 2. Functions of cocatalysts in photocatalytic $\mathrm{H}_{2}$ production.

harvesting. Some plasmon metals (such as $\mathrm{Au}$ and $\mathrm{Cu}$ ) can enhance visible light absorption due to the localized surface plasmon resonance (LSPR) effect [82,83]. (2) Cocatalysts can be effective in facilitating the separation of electron-hole pairs. When a cocatalyst is loaded on the surface of the photocatalysts, the cocatalyst can attract electrons, therefore the separation and transfer of the charge carriers can be enhanced $[11,84]$. (3) Cocatalysts can provide active sites for the photocatalytic reaction. Most cocatalysts can not only serve as electron sinks, but also provide proton reduction sites [85,86]. (4) Cocatalysts can improve the stability of the photocatalysts. Some metal sulfides can be oxidized easily. After loading cocatalysts, the self-decomposition of sulfides can be suppressed by extracting the photogenerated holes for oxygen evolution reac-
Table 1

The work functions of metals.

\begin{tabular}{lc}
\hline Metal & Work function $(\mathrm{eV})$ \\
\hline $\mathrm{Pt}$ & $5.65[109]$ \\
$\mathrm{Pd}$ & $5.2[110]$ \\
$\mathrm{Au}$ & $5.1[111]$ \\
$\mathrm{Ag}$ & $4.26[112]$ \\
$\mathrm{Ni}$ & $5.15[113]$ \\
$\mathrm{Co}$ & $5.0[114]$ \\
\hline
\end{tabular}

tions $[81,87]$.

\section{Single cocatalysts for photocatalytic $\mathrm{H}_{2}$ evolution}

\subsection{Metals and alloys}

Deposition of metals on the surface of semiconductors is one of the effective ways to improve the photocatalytic performance of photocatalysts. The photogenerated electrons migrate to the metal surface under the action of electric field formed by the relatively low Fermi level of the metal. The schematic of charge transfer for metal/semiconductor under light irradiation due to the existence of the Schottky junction is illustrated in Fig. 3(a). When a metal is in close contact with a semiconductor, Schottky barrier would be formed and resulted in band bending in the semiconductor at the interface of the contact, and the electrons must have a higher energy to over-

Table 2

Summary of metals as cocatalysts in photocatalyst systems for $\mathrm{H}_{2}$ evolution.

\begin{tabular}{|c|c|c|c|c|c|c|c|}
\hline Photocatalyst & Cocatalyst & Light source & $\begin{array}{l}\text { Weight } \\
\text { (mg) }\end{array}$ & Solution & Activity & $\mathrm{AQY}$ & Year/Ref. \\
\hline $\mathrm{Pt} / \mathrm{g}-\mathrm{C}_{3} \mathrm{~N}_{4}$ & $\mathrm{Pt}$ & 300 W Xe lamp & 50 & $200 \mathrm{~mL} 10$ vol\% TEOA & $318 \mu \mathrm{mol} \cdot \mathrm{h}^{-1}$ & $0.16 \%(420$ & $2016[90]$ \\
\hline 0.1 wt. $\% \mathrm{Pt} / \mathrm{g}-\mathrm{C}_{3} \mathrm{~N}_{4}$ & $\mathrm{Pt}$ & 300 W Xe lamp $(\lambda \geq 420 \mathrm{~nm})$ & 30 & $100 \mathrm{~mL} 10 \mathrm{vol} \%$ methanol & $473.82 \mu \mathrm{mol} \cdot \mathrm{mg}^{-1}$ & - & [54] \\
\hline $\mathrm{Pt} / \mathrm{g}-\mathrm{C}_{3} \mathrm{~N}_{4}$ & $\mathrm{Pt}$ & 300 W Xe lamp & 30 & $200 \mathrm{~mL} 10 \mathrm{vol} \%$ methanol & $42.1 \mu \mathrm{mol} \cdot \mathrm{h}^{-1}$ & - & 2018 [91] \\
\hline 1 wt. $\%$ Pt/g- ${ }_{3} \mathrm{~N}_{4}$ & $\mathrm{Pt}$ & 300 W Xe lamp $(\lambda \geq 420 \mathrm{~nm})$ & 100 & $100 \mathrm{~mL} 10 \mathrm{vol} \%$ TEOA & $34 \mu \mathrm{mol} \cdot \mathrm{h}^{-1}$ & - & 2015 [92] \\
\hline $\mathrm{Pt} / \mathrm{g}-\mathrm{C}_{3} \mathrm{~N}_{4}$ & $\mathrm{Pt}$ & $300 \mathrm{~W}$ Xe lamp $(\lambda \geq 420 \mathrm{~nm})$ & 50 & $80 \mathrm{~mL} 10 \mathrm{vol} \%$ TEOA & $571.01 \mu \mathrm{mol} \cdot \mathrm{h}^{-1} \cdot \mathrm{g}^{-1}$ & - & 2016 [93] \\
\hline $\mathrm{Pt} / \mathrm{g}-\mathrm{C}_{3} \mathrm{~N}_{4}$ & Pt & 300 W Xe lamp $(\lambda \geq 420 \mathrm{~nm})$ & 100 & $243 \mathrm{~mL} 10 \mathrm{vol} \% \mathrm{TEOA}$ & $417 \mu \mathrm{mol} \cdot \mathrm{h}^{-1} \cdot \mathrm{g}^{-1}$ & - & 2014 [94] \\
\hline 0.2 wt. $\% \mathrm{Pt} / \mathrm{g}-\mathrm{C}_{3} \mathrm{~N}_{4}$ & $\mathrm{Pt}$ & 300 W Xe lamp & 20 & $50 \mathrm{~mL} 10 \mathrm{vol} \%$ TEOA & $4875 \mu \mathrm{mol} \cdot \mathrm{g}^{-1} \cdot 4 \mathrm{~h}$ & - & 2017 [95] \\
\hline 1 wt. $\%$ Pt/g- $\mathrm{C}_{3} \mathrm{~N}_{4}$ & $\mathrm{Pt}$ & $350 \mathrm{~W}$ Xe lamp & 8 & $80 \mathrm{~mL} 10 \mathrm{vol} \%$ TEOA & $4210.8 \mu \mathrm{mol} \cdot \mathrm{h}^{-1} \cdot \mathrm{g}^{-1}$ & - & $2018[96]$ \\
\hline 1 wt. $\% \mathrm{Pd} / \mathrm{TiO}_{2}$ & $\mathrm{Pd}$ & $\begin{array}{c}200 \mathrm{~W} \text { SB-1000P/F lamp } \\
(\lambda \geq 365 \mathrm{~nm})\end{array}$ & 6.5 & $20 \mathrm{~mL} 10$ vol\% glycerol & $47.5 \mathrm{mmol} \cdot \mathrm{h}^{-1} \cdot \mathrm{g}^{-1}$ & - & [97] \\
\hline 0.5 wt. $\% \mathrm{Pd} / \mathrm{g}-\mathrm{C}_{3} \mathrm{~N}_{4}$ & $\mathrm{Pd}$ & 300 W Xe lamp $(\lambda \geq 400 \mathrm{~nm})$ & 20 & $100 \mathrm{~mL} 10$ & $1208.6 \mu \mathrm{mol} \cdot \mathrm{h}^{-1} \cdot \mathrm{g}^{-1}$ & $3.8 \%(420$ & [98] \\
\hline $0.5 \% \mathrm{Ag} / \mathrm{g}-\mathrm{C}_{3} \mathrm{~N}_{4}$ & $\mathrm{Ag}$ & 300 W Xe lamp $(\lambda \geq 400 \mathrm{~nm})$ & 50 & $200 \mathrm{~mL} 10$ vol\% methanol & $18.09 \mu \mathrm{mol} \cdot \mathrm{h}^{-1} \cdot \mathrm{g}^{-1}$ & - & $2016[100]$ \\
\hline 20 wt. $\% \mathrm{Ag} / \mathrm{MoS}_{2}$ & $\mathrm{Ag}$ & 300 W Xe lamp & 2.1 & $\begin{array}{c}4.5 \mathrm{~mL} 0.3 \mathrm{M} \mathrm{Na}_{2} \mathrm{~S} / 0.3 \mathrm{M} \\
\mathrm{Na}_{2} \mathrm{SO}_{3}\end{array}$ & $179.5 \mu \mathrm{mol} \cdot \mathrm{g}^{-1}$ & - & 2015 [102] \\
\hline 3 wt. $\%$ Ag/g- $\mathrm{C}_{3} \mathrm{~N}_{4}$ & Ag & 300 W Xe lamp & 5 & $70 \mathrm{~mL} 10$ vol\% TEOA & $2014.2 \mu \mathrm{mol} \cdot \mathrm{h}^{-1} \cdot \mathrm{g}^{-1}$ & - & [103] \\
\hline 2 wt. $\% \mathrm{Au} / \mathrm{g}-\mathrm{C}_{3} \mathrm{~N}_{4}$ & $\mathrm{Au}$ & $300 \mathrm{~W}$ Xe lamp $(\lambda \geq 420 \mathrm{~nm})$ & 20 & $40 \mathrm{~mL} 2.5$ vol $\%$ TEOA & $540 \mu \mathrm{mol} \cdot \mathrm{h}^{-1} \cdot \mathrm{g}^{-1}$ & $1.22 \%(420 \mathrm{~nm})$ & $2018[104]$ \\
\hline $\mathrm{Au} / \mathrm{TiO}_{2}$ & $\mathrm{Au}$ & 300 W Xe lamp & 25 & $50 \mathrm{~mL} 20 \mathrm{vol} \%$ TEOA & $4.92 \mathrm{mmol} \cdot \mathrm{h}^{-1} \cdot \mathrm{g}^{-1}$ & - & $2018[24]$ \\
\hline $\mathrm{Au} / \mathrm{TiO}_{2}$ & $\mathrm{Au}$ & $00 \mathrm{~W}$ Xe lamp $(\lambda \geq 420 \mathrm{~nm})$ & 10 & $20 \mathrm{~mL} 20$ vol\% methanol & $12.4 \mu \mathrm{mol} \cdot \mathrm{h}^{-1} \cdot \mathrm{g}^{-1}$ & - & [107] \\
\hline $\mathrm{Au} / \mathrm{g}-\mathrm{C}_{3} \mathrm{~N}_{4}$ & $\mathrm{Au}$ & 300 W Xe lamp $(\lambda \geq 420 \mathrm{~nm})$ & 50 & $100 \mathrm{~mL} 10 \mathrm{vol} \%$ TEOA & $84.1 \mu \mathrm{mol} \cdot \mathrm{h}^{-1}$ & - & 2018 [108] \\
\hline $\mathrm{Zn} / \mathrm{g}-\mathrm{C}_{3} \mathrm{~N}_{4}$ & $\mathrm{Zn}$ & $200 \mathrm{~W}$ Xe lamp $(\lambda \geq 420 \mathrm{~nm})$ & 200 & $270 \mathrm{~mL} 18.5$ vol\% methanol & $59.5 \mu \mathrm{mol} \cdot \mathrm{h}^{-1}$ & $3.2 \%(420 \mathrm{~nm})$ & $2011[125]$ \\
\hline 0.5 wt. $\% \mathrm{Ni} / \mathrm{TiO}_{2}$ & $\mathrm{Ni}$ & $\begin{array}{c}100 \text { W SB-100P/F lamp } \\
(\lambda \geq 365 \mathrm{~nm})\end{array}$ & 6.5 & $20 \mathrm{~mL} 5$ vol\% EtOH & $24.3 \mathrm{mmol} \cdot \mathrm{h}^{-1} \cdot \mathrm{g}^{-1}$ & - & 201 \\
\hline $10 \% \mathrm{Ni} / \mathrm{g}-\mathrm{C}_{3} \mathrm{~N}_{4}$ & $\mathrm{Ni}$ & $500 \mathrm{~W}$ Xe arc lamp & 50 & $100 \mathrm{~mL} 10$ vol\% TEOA & $8.41 \mu \mathrm{mol} \cdot \mathrm{h}^{-1}$ & 一 & 2015 [120] \\
\hline $7.40 \% \mathrm{Ni} / \mathrm{g}-\mathrm{C}_{3} \mathrm{~N}_{4}$ & & 300 W Xe lamp & 10 & $10 \mathrm{~mL} 30$ vol$\%$ TEOA & $4318 \mu \mathrm{mol} \cdot \mathrm{h}^{-1} \cdot \mathrm{g}^{-1}$ & $2.1 \%(400 \mathrm{~nm})$ & $2016[121]$ \\
\hline 3 wt. $\% \mathrm{Ni} / \mathrm{Cd} \operatorname{In}_{2} \mathrm{~S}_{4}$ & $\mathrm{Ni}$ & $100 \mathrm{~W}$ solar simulator & 5 & $10 \mathrm{~mL} 10 \mathrm{vol} \%$ TEOA & $1060 \mu \mathrm{mol} \cdot \mathrm{h}^{-1} \cdot \mathrm{g}^{-1}$ & - & 2017 [122] \\
\hline 5 wt. $\% \mathrm{Ni} / \mathrm{CdS}$ & $\mathrm{Ni}$ & $300 \mathrm{~W}$ Xe lamp $(\lambda \geq 400 \mathrm{~nm})$ & 100 & $50 \mathrm{~mL} 50$ vol\% lactic acid & $3004.8 \mu \mathrm{mol} \cdot \mathrm{h}^{-1}$ & - & 2014 [123] \\
\hline $10 \% \mathrm{Co} / \mathrm{g}-\mathrm{C}_{3} \mathrm{~N}_{4}$ & Co & 300 W Xe lamp & 20 & $15 \mathrm{~mL} 20 \mathrm{vol} \%$ TEOA & $11.48 \mu \mathrm{mol} \cdot \mathrm{h}^{-1}$ & $5.34 \%(400 \mathrm{~nm})$ & $2018[124]$ \\
\hline $2.5 \% \mathrm{Cu} / \mathrm{TiO}_{2}$ & $\mathrm{Cu}$ & $300 \mathrm{~W}$ Xe lamp & 50 & $100 \mathrm{~mL} 20 \mathrm{vol} \%$ methanol & $6046 \mu \mathrm{mol} \cdot \mathrm{h}^{-1} \cdot \mathrm{g}^{-1}$ & - & 2019 [28] \\
\hline 0.3 wt. $\% \mathrm{Cu} / \mathrm{TiO}_{2}$ & $\mathrm{Cu}$ & $300 \mathrm{~W}$ Xe lamp $(\lambda \geq 300 \mathrm{~nm})$ & 50 & $65 \mathrm{~mL} 20$ vol\% methanol & $20.4 \mathrm{mmol} \cdot \mathrm{h}^{-1} \cdot \mathrm{g}^{-1}$ & - & $2018[23]$ \\
\hline 1 wt. $\% \mathrm{Cu} / \mathrm{Nb}_{2} \mathrm{O}_{5}$ & $\mathrm{Cu}$ & $\begin{array}{l}400 \mathrm{~W} \text { medium pressure } \\
\text { halide lamp }(\lambda=360 \mathrm{~nm})\end{array}$ & 200 & $550 \mathrm{~mL} 16.7$ vol\% methanol & $1572 \mu \mathrm{mol} \cdot \mathrm{h}^{-1} \cdot \mathrm{g}^{-1}$ & - & $2011[126]$ \\
\hline
\end{tabular}


(a)

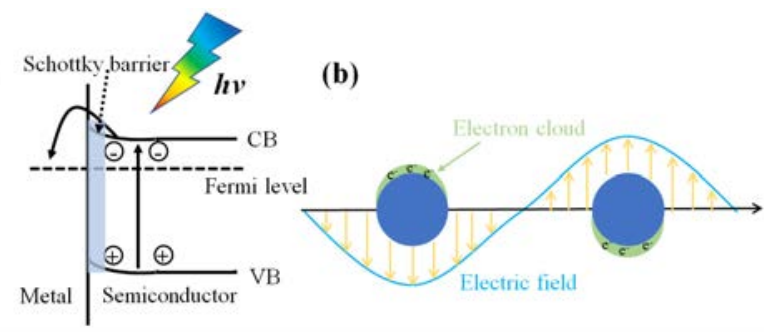

Fig. 3. Schematic of (a) Schottky junction and (b) LSPR effect.

come the barrier $[81,88,89]$. The photogenerated electrons will transfer from the CB of the semiconductor to the metal through the Schottky barrier until their Fermi levels are equal, and the holes will hold in the VB of the semiconductor, thereby achieving effective separation of $e^{--} h^{+}$pairs. The metal not only acts as a cocatalyst to separate photogenerated electrons-holes, but also provides an active site for the photocatalytic $\mathrm{H}_{2}$ evolution. In this section, the application of transition metal phosphides materials as cocatalysts for photocatalytic $\mathrm{H}_{2}$ production is introduced, and Table 2 and Table 3 summarize the application of metals and alloys as cocatalysts in the photocatalyst system for $\mathrm{H}_{2}$ evolution.

Several noble-metals such as Pt [54,90-96], Pd [97-99], Ag [100-103] and $\mathrm{Au}[24,104-108]$ have been investigated in recent years. Pt, with the largest work function among many noble-metal, (Table 1), is the best cocatalyst for trapping electrons.

In 2016, Li et al. [90] reported that isolated single Pt atoms as a new form of cocatalysts deposited on $\mathrm{g}-\mathrm{C}_{3} \mathrm{~N}_{4}$ to enhance the photocatalytic $\mathrm{H}_{2}$ evolution activity. Under visible-light irradiation, bare g- $\mathrm{C}_{3} \mathrm{~N}_{4}$ gives a low activity with $\mathrm{H}_{2}$ evolution rate of $6.5 \mu \mathrm{mol} \cdot \mathrm{h}^{-1}$. While with $0.16 \mathrm{wt} . \%$ Pt loading, Pt-CN shows a $\mathrm{H}_{2}$ generation rate up to $318 \mu \mathrm{mol} \cdot \mathrm{h}^{-1}$. The photocatalytic performance of $\mathrm{H}_{2}$ production can be improved by adding isolated single $\mathrm{Pt}$ atom to the intrinsic change of the $\mathrm{g}-\mathrm{C}_{3} \mathrm{~N}_{4}$ surface trap state induced by Pt atoms. Zhu et al. [54] concluded that the size of Pt co-catalyst can be controlled to form single atoms by inducing a small amount of Pt precursor, which plays a pivotal role in enhancing the photocatalytic performance of g- $\mathrm{C}_{3} \mathrm{~N}_{4}$. As shown in Fig. 4, the average size of Pt on $\mathrm{g}-\mathrm{C}_{3} \mathrm{~N}_{4}$ is less than $0.2 \mathrm{~nm}$ after loading $0.1 \mathrm{wt} . \% \mathrm{Pt}$. With increasing the loading amount of Pt, the size of Pt clusters is increased. When the size of the Pt clusters is the smallest, the photocatalytic $\mathrm{H}_{2}$ generation rate of $\mathrm{Pt}_{0.1}-\mathrm{CN}$ is $473.82 \mu \mathrm{mol} \cdot \mathrm{mg}^{-1}$ under the visible-light irradiation. It is ascribed to the enhanced utilization efficiency of Pt atoms.

Liu et al. [96] synthetized Pt/g- $\mathrm{C}_{3} \mathrm{~N}_{4}$ by changing the sequence of adding TEOA on photo-deposition of Pt. The sample named CNS-Pt-A and CNS-Pt-B that TEOA is added into the photocatalytic system after and before photo-deposition of $\mathrm{Pt}$, respectively. Both CNS-Pt-A and CNS-Pt-B show better photocatalytic $\mathrm{H}_{2}$ production activity as shown in Fig. 5, and the CNS-Pt-A shows a higher photocatalytic $\mathrm{H}_{2}$ generation rate of $4210.8 \mu \mathrm{mol} \cdot \mathrm{h}^{-1} \cdot \mathrm{g}^{-1}$, which is attributed to the formation of coordination complex between TEOA and $\mathrm{Pt}^{4+}$, inhibiting the photo-reduction of $\mathrm{Pt}^{4+}$ to metallic $\mathrm{Pt}$.

Metals are modified on the surface of semiconductors to form metal/semiconductor systems, which act as co-catalysts

Table 3

Summary of metal alloys as cocatalysts photocatalyst system for $\mathrm{H}_{2}$ evolution.

\begin{tabular}{|c|c|c|c|c|c|c|c|}
\hline Photocatalyst & Cocatalyst & Light source & $\begin{array}{l}\text { Weight } \\
\text { (mg) }\end{array}$ & Solution & Activity & $\mathrm{AQY}$ & Year/Ref. \\
\hline 0.2 wt. $\% \mathrm{Pt}_{0.5} \mathrm{Pd}_{0.5} / \mathrm{g}-\mathrm{C}_{3} \mathrm{~N}_{4}$ & $\mathrm{Pt}_{0.5} \mathrm{Pd}_{0.5}$ & $300 \mathrm{~W}$ Xe lamp $(\lambda \geq 400 \mathrm{~nm})$ & 50 & $\begin{array}{l}100 \mathrm{~mL} 10 \mathrm{vol}^{2} \% \\
\text { TEOA/K2 } \mathrm{HPO}_{4}\end{array}$ & $1600.8 \mu \mathrm{mol} \cdot \mathrm{h}^{-1} \cdot \mathrm{g}^{-1}$ & $\begin{array}{c}5.5 \% \\
(420 \mathrm{~nm})\end{array}$ & 2019 [127] \\
\hline 1.0 wt. $\%$ PtCo $/ g-C_{3} \mathrm{~N}_{4}$ & PtCo & $300 \mathrm{~W}$ Xe lamp $(\lambda \geq 400 \mathrm{~nm})$ & 50 & $100 \mathrm{~mL} 10$ vol $\%$ TEOA & $960 \mu \mathrm{mol} \cdot \mathrm{h}^{-1} \cdot \mathrm{g}^{-1}$ & - & 2015 [128] \\
\hline 0.5 wt. $\% A u P d / g-C_{3} \mathrm{~N}_{4}$ & AuPd & $300 \mathrm{~W}$ Xe lamp $(\lambda \geq 400 \mathrm{~nm})$ & 50 & $100 \mathrm{~mL} 10$ vol\% TEOA & $326 \mu \mathrm{mol} \cdot \mathrm{h}^{-1} \cdot \mathrm{g}^{-1}$ & - & 2015 [129] \\
\hline 1.0 wt. $\%$ AuPd/g- ${ }_{3} \mathrm{~N}_{4}$ & AuPd & 300 W Xe lamp $(\lambda \geq 400 \mathrm{~nm})$ & 50 & $\begin{array}{l}100 \mathrm{~mL} 10 \text { vol} \% \\
\text { TEOA/K } / \mathrm{HPO}_{4}\end{array}$ & $107 \mu \mathrm{mol} \cdot \mathrm{h}^{-1}$ & - & $2017[130]$ \\
\hline $\mathrm{AgCu}(3) / \mathrm{C}_{3} \mathrm{~N}_{4} \mathrm{NTs}$ & $\mathrm{AgCu}$ & 300 W Xe lamp $(\lambda \geq 420 \mathrm{~nm})$ & 40 & $40 \mathrm{~mL} 10 \mathrm{vol} \%$ TEOA & $738 \mu \mathrm{mol} \cdot \mathrm{g}^{-1}$ & - & 2018 [131] \\
\hline $\mathrm{NiCd} / \mathrm{CdS}$ & $\mathrm{NiCd}$ & $300 \mathrm{~W}$ Xe lamp $(\lambda \geq 410 \mathrm{~nm})$ & 20 & $100 \mathrm{~mL} 0.1 \mathrm{M} \mathrm{Na}_{2} \mathrm{~S} / \mathrm{Na}_{2} \mathrm{SO}_{3}$ & $11.57 \mathrm{mmol} \cdot \mathrm{h}^{-1} \cdot \mathrm{g}^{-1}$ & - & 2019 [132] \\
\hline 10 wt. $\% \mathrm{Ni}_{0.4} \mathrm{Mo}_{0.6} / \mathrm{g}-\mathrm{C}_{3} \mathrm{~N}_{4}$ & $\mathrm{Ni}_{0.4} \mathrm{Mo}_{0.6}$ & $300 \mathrm{~W}$ Xe lamp $(\lambda \geq 420 \mathrm{~nm})$ & 20 & $100 \mathrm{~mL} 10$ vol $\%$ TEOA & $1785 \mu \mathrm{mol} \cdot \mathrm{h}^{-1} \cdot \mathrm{g}^{-1}$ & $\begin{array}{c}0.05 \% \\
(420 \mathrm{~nm})\end{array}$ & 2019 [133] \\
\hline $\mathrm{Ni}_{0.8} \mathrm{Co}_{0.2} / \mathrm{Zn}_{0.75} \mathrm{Cd}_{0.25} \mathrm{~S}$ & $\mathrm{Ni}_{0.8} \mathrm{Co}_{0.2}$ & $300 \mathrm{~W}$ Xe lamp $(\lambda \geq 420 \mathrm{~nm})$ & 50 & $\begin{array}{c}100 \mathrm{~mL} 10 \mathrm{vol} \% \\
\text { TEOA/0.35 } \mathrm{M} \mathrm{Na} 2 \mathrm{~S} / 0.25 \mathrm{M} \\
\mathrm{Na}_{2} \mathrm{SO}_{3}\end{array}$ & $211.4 \mathrm{mmol} \cdot \mathrm{h}^{-1} \cdot \mathrm{g}^{-1}$ & $\begin{array}{c}13.3 \% \\
(365 \mathrm{~nm})\end{array}$ & 2018 [134] \\
\hline 1 wt. $\% \mathrm{Ni}_{1}-\mathrm{Pd}_{10} / \mathrm{TiO}_{2}$ & $\mathrm{Ni}_{1}-\mathrm{Pd}_{10}$ & $\begin{array}{c}400 \mathrm{~W} \text { mercury arc UV-vis } \\
\text { irradiation }\end{array}$ & 50 & $5 \mathrm{~mL} 50 \mathrm{vol} \%$ methanol & $1050 \mu \mathrm{mol} \cdot \mathrm{h}^{-1} \cdot \mathrm{g}^{-1}$ & - & $2017[136]$ \\
\hline 0.5 wt. $\% \mathrm{Ni}_{5}-\mathrm{Au}_{1} / \mathrm{TiO}_{2}$ & $\mathrm{Ni}_{5}-\mathrm{Au}_{1}$ & 300 W Xe lamp & 30 & $3 \mathrm{~mL} 50$ vol\% methanol & $937.2 \mu \mathrm{mol} \cdot \mathrm{h}^{-1} \cdot \mathrm{g}^{-1}$ & - & 2016 [137] \\
\hline 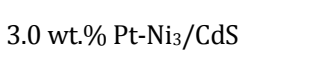 & $\mathrm{Pt}-\mathrm{Ni}_{3}$ & 300 W Xe lamp $(\lambda \geq 420 \mathrm{~nm})$ & 100 & $50 \mathrm{~mL} 1.0 \mathrm{M}\left(\mathrm{NH}_{4}\right)_{2} \mathrm{SO}_{3}$ & $48.96 \mathrm{mmol} \cdot \mathrm{h}^{-1} \cdot \mathrm{g}^{-1}$ & $\begin{array}{c}44.0 \% \\
(420 \mathrm{~nm})\end{array}$ & $2018[36]$ \\
\hline $\mathrm{Pt}-\mathrm{Ni} / \mathrm{g}-\mathrm{C}_{3} \mathrm{~N}_{4}$ & Pt-Ni & $300 \mathrm{~W}$ Xe lamp $(\lambda \geq 420 \mathrm{~nm})$ & 50 & $100 \mathrm{~mL} 10$ vol $\%$ TEOA & $104.7 \mu \mathrm{mol} \cdot \mathrm{h}^{-1} \cdot \mathrm{g}^{-1}$ & - & 2018 [138] \\
\hline $\begin{array}{l}0.5 \text { wt. } \% \text { Ag- } 2 \text { wt. } \% \\
\mathrm{Cu} / \mathrm{TiO}_{2}\end{array}$ & $\mathrm{AgCu}$ & artificial Sun A.M1.5 & 10 & $50 \mathrm{~mL} 10 \mathrm{vol} \%$ methanol & $5683 \mu \mathrm{mol} \cdot \mathrm{h}^{-1} \cdot \mathrm{g}^{-1}$ & - & 2016 [139] \\
\hline 0.5 wt. $\%$ Au4Cu1/CaIn ${ }_{2} \mathrm{~S}_{4}$ & $\mathrm{Au} 4 \mathrm{Cu} 1$ & $300 \mathrm{~W}$ Xe lamp $(\lambda \geq 420 \mathrm{~nm})$ & 10 & $\begin{array}{c}100 \mathrm{~mL} 3.15 \mathrm{~g} \mathrm{Na}_{2} \mathrm{SO}_{3} / 6.0 \mathrm{~g} \\
\mathrm{Na}_{2} \mathrm{~S} \cdot 9 \mathrm{H}_{2} \mathrm{O}\end{array}$ & $452.8 \mu \mathrm{mol} \cdot \mathrm{h}^{-1}$ & - & 2018 [135] \\
\hline 0.5 wt.\% Pt0.5-Au1/ $\mathrm{TiO}_{2}$ & Pt0.5Au1 & $300 \mathrm{~W}$ Xe lamp $(\lambda \geq 400 \mathrm{~nm})$ & 20 & $30 \mathrm{~mL} 25$ vol\% methanol & $311 \mu \mathrm{mol} \cdot \mathrm{h}^{-1} \cdot \mathrm{g}^{-1}$ & $\begin{array}{c}1.6 \% \\
(400 \mathrm{~nm})\end{array}$ & 2015 [140] \\
\hline $7 \% \mathrm{Cu}_{63} \mathrm{Ni}_{37} / \mathrm{TiO}_{2}$ & $\mathrm{Cu}_{63} \mathrm{Ni}_{37}$ & Xe lamp & 100 & 100 mL 10 vol\% methanol & $0.63 \mathrm{mmol} \cdot \mathrm{h}^{-1}$ & - & 2017 [141] \\
\hline 1 wt. $\% \mathrm{CuNi} / \mathrm{rGO}$ & $\mathrm{CuNi}$ & 300 W Xe lamp & 5 & $70 \mathrm{~mL} 14.2$ vol\% lactic acid & $1787 \mu \mathrm{mol} \cdot \mathrm{h}^{-1} \cdot \mathrm{g}^{-1}$ & - & 2017 [43] \\
\hline
\end{tabular}



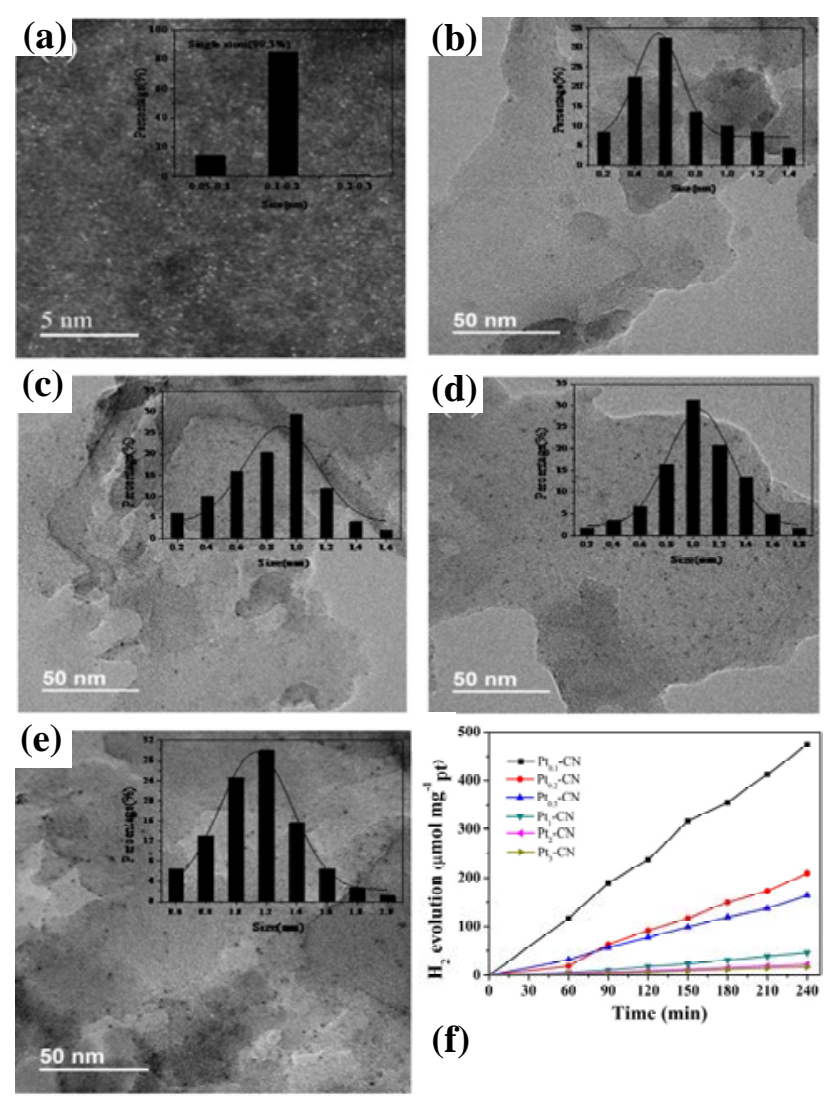

Fig. 4. Aberration-corrected STEM images of Pt0.1-CN (a) and HRTEM of Pt0.3-CN (b), Pt1-CN (c), Pt2-CN (d), and Pt3-CN (e). Time course of hydrogen evolution over $4 \mathrm{~h}$ for $\mathrm{Pt}_{\mathrm{x}}-\mathrm{CN}$ with different Pt contents (f). From Ref. [54].

to provide active sites and trapping electrons to separate photoinduced electron-hole pairs. When the frequency of the incident photons matches well with the inherent frequency of the free electrons on the surface of metal nanoparticles, which will lead to a collective oscillation, and the metal nanoparticles will have a strong absorption of photon energy, this effect is commonly known as localized surface plasmon resonance (LSPR) as shown in Fig. 2(b) $[89,115]$. As previously mentioned, the light absorption ability of photocatalyst is an important factor that affects the photocatalytic hydrogen production, the more light that is absorbed, the more photo-generated charges are produced to enhance the rate of photocatalytic $\mathrm{H}_{2}$ evolution. $\mathrm{Au}$ $[24,105]$ and $\mathrm{Cu}[116,117]$ are the most popular materials used for plasmonic cocatalysts to enhance light absorption.

Guo et al. [104] reported $\mathrm{Au}$ as cocatalyst on $\mathrm{g}-\mathrm{C}_{3} \mathrm{~N}_{4}$ nanosheets with Au nanospheres (NSs) and nanorods (NRs) of different plasmon resonance wavelengths. Fig. 6a shows the SEM and TEM images of the Au nanocrystals on $\mathrm{g}-\mathrm{C}_{3} \mathrm{~N}_{4}$ nanosheets with Au loading amount of $2.0 \mathrm{wt} . \%$. With the size of the $\mathrm{Au}$ nanocrystals increasing, the plasmon peak of the $\mathrm{Au} / \mathrm{g}-\mathrm{C}_{3} \mathrm{~N}_{4}$ sample moves toward the long wavelength due to the reduction of the effective refractive index of the surrounding environment. The optimal $18 \mathrm{~nm}$-sized $\mathrm{Au}$ nanospheres/g- $\mathrm{C}_{3} \mathrm{~N}_{4}$ nanostructure exhibits a superior activity, with the $\mathrm{H}_{2}$ generation rate reaching $540 \mu \mathrm{mol} \cdot \mathrm{h}^{-1} \cdot \mathrm{g}^{-1}$ under visible light. The introduced plasmonic Au nanocrystals not only enhance the photocatalytic activity, but also endow the hybrid photocatalysts with an extended light absorption range. Meanwhile, the improved $\mathrm{H}_{2}$ production rates can be attributed to the LSPR effect of Au.

Although noble metal cocatalysts own high photocatalytic activity, their practical applications are limited by some factors such as resource shortages and high prices. Non-noble metals are rich in the earth's crust and some of their properties are similar to noble metals in enhancing the photocatalytic performance. Furthermore, non-noble metals also possess high work function as noble metals (Table 2), therefore non-noble metals play an important role as co-catalysis to trap and transfer electrons in photocatalytic reaction. Recently, non-noble metals such as Zn [118], Ni [119-123] and Co [124] have been utilized to effectively driven photocatalytic water splitting. Among the non-noble metals, the work function of $\mathrm{Ni}$ is closest to $\mathrm{Pt}$, even higher than $\mathrm{Au}$, therefore $\mathrm{Ni}$ is the most promising substitute for noble metals as co-catalysts for photocatalytic $\mathrm{H}_{2}$ evolution. In 2016, Dong's group [121] successfully synthetized $\mathrm{Ni} / g-\mathrm{C}_{3} \mathrm{~N}_{4}$ photocatalyst, in which the non-noble metal $\mathrm{Ni}$ as cocatalyst was loaded on the surface of g- $\mathrm{C}_{3} \mathrm{~N}_{4}$ nanosheets for photocatalytic water splitting under sun-light irradiation. In the absence of cocatalyst, the pure $\mathrm{g}-\mathrm{C}_{3} \mathrm{~N}_{4}$ nanosheets showed a low activity of photocatalytic hydrogen evolution due to the strong recombination trend of electrons and holes. When the loading content of $\mathrm{Ni}$ was $7.40 \mathrm{wt} . \%$, the rate of $\mathrm{H}_{2}$ evolution was $4318 \mu \mathrm{mol} \cdot \mathrm{h}^{-1} \cdot \mathrm{g}^{-1}$ with $2.10 \% \mathrm{AQY}$ at wavelength of 400 $\mathrm{nm}$, and kept stable after $48 \mathrm{~h}$ of irradiation. It is worth noting that the content of $\mathrm{Ni}^{2+}$ in the photocatalytic system $\mathrm{B}$ is the same as that in photocatalytic system A, as shown in Fig. 6 a. Comparing with the rate of $\mathrm{H}_{2}$ evolution, the photocatalytic activity of system A was 2.45 times higher than that of system
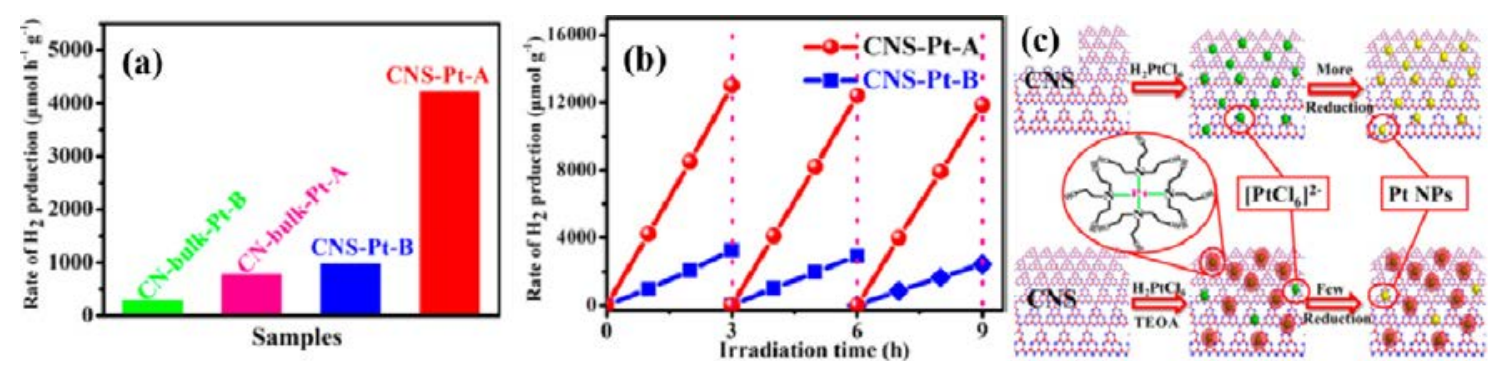

Fig. 5. (a) Photocatalytic $\mathrm{H}_{2}$-production activity of CN-bulk-Pt-A, CN-bulk-Pt-B, CNS-Pt-B, and CNS-Pt-A in 10 vol\% TEOA under the irradiation of 350 W Xe lamp; (b) Cyclic $\mathrm{H}_{2}$-production experiments of CNS-Pt-B and CNS-Pt-A and the corresponding $\mathrm{H}_{2}$-production rate for the two samples; (c) Diagrammatic sketch of the photoreduction process of Pt with TEOA adding into the system after or before the photo-deposition of Pt. From Ref. [96]. 


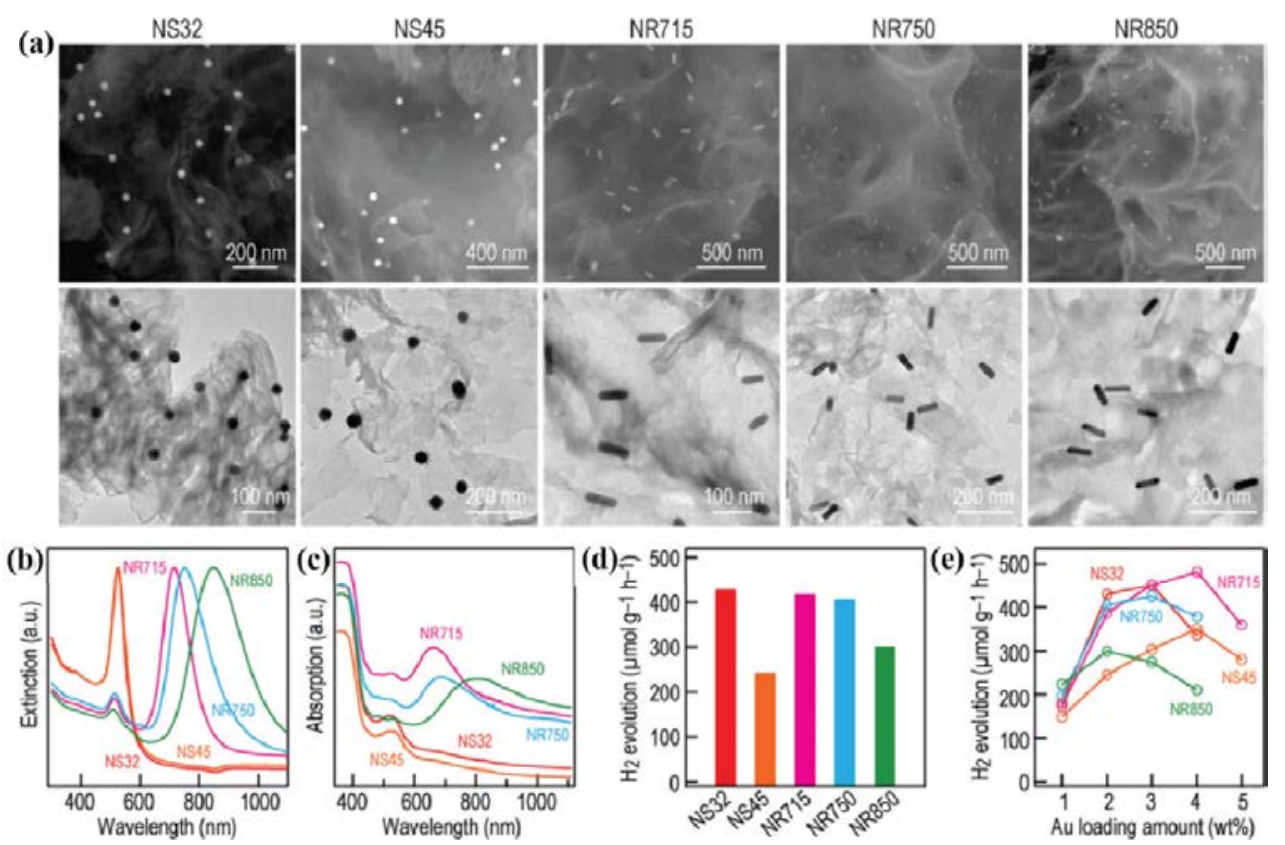

Fig. 6. Characterization and $\mathrm{H}_{2}$ evolution of the Au nanocrystals/g- $\mathrm{C}_{3} \mathrm{~N}_{4}$ samples. (a) SEM (upper row) and TEM (lower row) images of the Au nanocrystals/g- $\mathrm{C}_{3} \mathrm{~N}_{4}$ samples with an Au loading amount of $2.0 \mathrm{wt}$.\%. The numbers on the top represent the names of the Au nanocrystal samples. (b) Extinction spectra of the $\mathrm{Au}$ nanocrystals in aqueous solutions. (c) Absorption spectra of the corresponding hybrid nanostructure samples. (d) $\mathrm{H}_{2}$ generation rates for the different Au nanocrystals/g- $\mathrm{C}_{3} \mathrm{~N}_{4}$ samples with the same Au loading amount of 2.0 wt.\%. (e) Variations of the $\mathrm{H}_{2}$ evolution rates for the different $\mathrm{Au}$ nanocrystals/g- $\mathrm{C}_{3} \mathrm{~N}_{4}$ samples as functions of the Au loading amount. From Ref. [104].

B. The reason for this phenomenon is that $\mathrm{Ni}^{2+}$ and $\mathrm{TEOA}$ (as sacrifice reagent) could generate a new compound $\left[\mathrm{Ni}(\mathrm{TEOA})_{2}\right] \mathrm{Cl}_{2}$, which acts as the main co-catalyst, not metal $\mathrm{Ni}$ in the case of photocatalytic system B. Therefore, non-noble metal $\mathrm{Ni}$ could be helpful for the transfer of photogenerated electrons and high efficiency of photocatalytic $\mathrm{H}_{2}$ evolution.

Metallic copper is common in our lives because of its earth-abundance and have been used as co-catalyst in different photocatalytic reactions. Some research groups reported that $\mathrm{Cu}$ nanoparticles loading on some semiconductors formed Schottky contact. To date, Cheng and co-worker [28] developed a highly efficient photocatalysis system for water splitting by loading metal $\mathrm{Cu}$ on $\mathrm{TiO}_{2}$ microrods. As shown in Fig. 7(a, b), the $\mathrm{TiO}_{2}$-Cu- 2.5 wt.\% hybrid photocatalyst exhibited superior
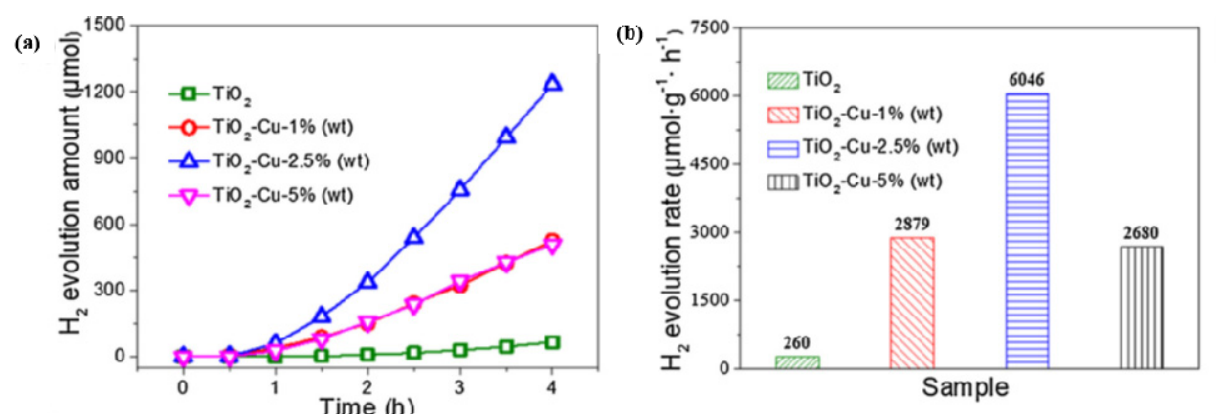

(c) Beforctis

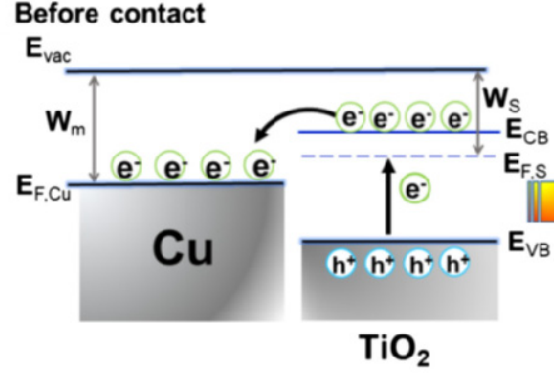

(d) After contact

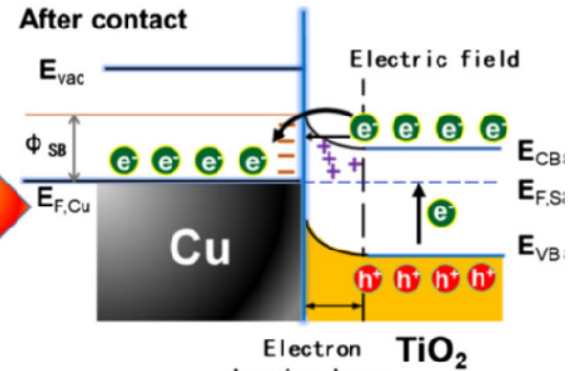

barrier Iayer

Fig. 7. (a) Photocatalytic $\mathrm{H}_{2}$ evolution of $\mathrm{Cu}$ loaded on $\mathrm{TiO}_{2}$ at different loading, and (b) average $\mathrm{H}_{2}$ generation rate. Energy band diagrams of metal $\mathrm{Cu}$ and $\mathrm{TiO}_{2}$, and the proposed electron transfer path in the photocatalytic reaction: (c) before contact and (d) after contact. $E_{\mathrm{vac}}$, vacuum energy; $E_{\mathrm{v}}$, energy of the valence band maximum; $E_{\mathrm{c}}$, energy of the conduction band minimum; $E_{\mathrm{F}, \mathrm{S}}$, Fermi level of the semiconductor; $W_{\mathrm{s}}$, semiconductor work function; $W_{\mathrm{m}}$, metal work function; $\oint_{\mathrm{SB}}$, Schottky barrier. From Ref. [28]. 

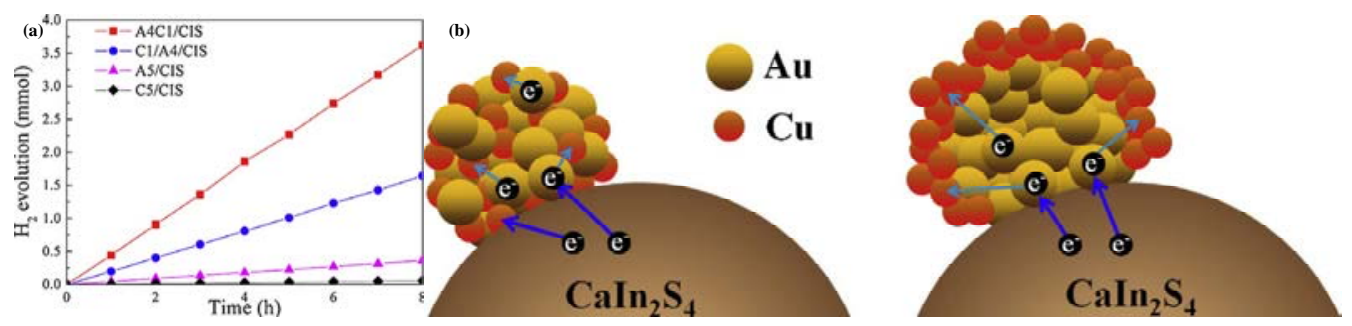

Fig. 8. (a) Photocatalytic hydrogen production for A5/CIS, C5/CIS, C1/A4/CIS and A4C1/CIS composites under visible light irradiation; (b) Schematic illustration for electron transfer mechanism in AuCu bimetallic nanoparticles under visible light irradiation. From Ref. [135].

rate of hydrogen evolution with $6046 \mu \mathrm{mol} \cdot \mathrm{h}^{-1} \cdot \mathrm{g}^{-1}$, which is 23 times higher than that of pure $\mathrm{TiO}_{2}$ microrods. The significant increase in photocatalytic performance is due to the Schottky junction formed between metal $\mathrm{Cu}$ and $\mathrm{TiO}_{2}$ microrods as shown in Fig. 7(c, d). The work function of $\mathrm{Cu}$ is larger than that of $\mathrm{TiO}_{2}$, the $e^{-}$would transfer from $\mathrm{TiO}_{2}$ to $\mathrm{Cu}$. The movement of electrons causes the Fermi levels of metallic $\mathrm{Cu}$ and $\mathrm{TiO}_{2}$ to reach the same level. In the equilibrium state, a depletion layer is formed at the $\mathrm{TiO}_{2}-\mathrm{Cu}$ interface, wherein the $\mathrm{Cu}$ metal is negatively charged, and $\mathrm{TiO}_{2}$ is positively charged due to electrostatic induction near its surface. The electric field would be formed between $\mathrm{Cu}$ and $\mathrm{TiO}_{2}$, the photoexcited electrons would be difficult to pass through the Schottky barrier to recombination with holes, so that the electrons and holes could be efficiently separated to improve the rate of $\mathrm{H}_{2}$ evolution. Therefore, metal $\mathrm{Cu}$ which is low-cost and earth-abundant could replace the noble metals as co-catalyst for photocatalytic water splitting.

Similar to mono-metallic, metal alloys such as PtPd [127], PtCo [128], AuPd [129,130], AgCu [131], NiCd [132], NiMo [133] and NiCo [134] have also been synthesized and reported in the field of photocatalytic $\mathrm{H}_{2}$ evolution, which act as co-catalysts to trap electrons from the semiconductor and improve the charge separation and transfer efficiency [89]. Compared with single-metal, alloy co-catalysts commonly show higher activity in photocatalytic $\mathrm{H}_{2}$ evolution, which is mainly due to the synergistic effect between the two metals [135]. Bimetallic cocatalysts such as AuCu [135] and AuPd [129,130], which contain plasmonic metals also possess LSPR effect.

In alloy NPs, the activity could be influenced by the different atomic arrangements, as exhibited in Fig. 8(a). Ding and co-workers [135] reported that the photocatalytic $\mathrm{H}_{2}$ evolution rate of $\mathrm{AuCu}$ alloy nanoparticles/CaIn $\mathrm{S}_{4}$ composite is up to $452.8 \mu \mathrm{mol} \cdot \mathrm{h}^{-1}$, which is 2.2 times higher than the $\mathrm{AuCu}$ core-shell structure/CaIn ${ }_{2} \mathrm{~S}_{4}$ composite. The catalytic mechanism for charge separation is outlined in Fig. 8(b). The photocatalytic performance enhancement of $\mathrm{AuCu}$ alloy nanoparticles is better than that of $\mathrm{AuCu}$ core-shell structure due to the shorter distance of photoelectrons transfer from $\mathrm{CaIn}_{2} \mathrm{~S}_{4}$ to $\mathrm{Au}$, thus to $\mathrm{Cu}$. Moreover, the existence of $\mathrm{Cu}$ shell in $\mathrm{AuCu}$ core-shell nanoparticles may decrease the visible absorption of $\mathrm{Au}$ core because the incident light can be scattered by $\mathrm{Cu}$ shell. Therefore, the AuCu alloy nanoparticles as cocatalyst is better than that of AuCu core-shell structure.

Our group [127] reported that PtPd alloy nanoparticles as cocatalyst were deposited on $\mathrm{g}-\mathrm{C}_{3} \mathrm{~N}_{4}$ nanosheets to enhance the photocatalytic $\mathrm{H}_{2}$ evolution activity under visible-light irradiation as shown in Fig. 9. The 0.2 wt.\% $\mathrm{Pt}_{0.5} \mathrm{Pd}_{0.5} / \mathrm{g}-\mathrm{C}_{3} \mathrm{~N}_{4}$ sample presented the highest $\mathrm{H}_{2}$ evolution activity (1600.8 $\mu \mathrm{mol} \cdot \mathrm{g}^{-1} \cdot \mathrm{h}^{-1}$ ), which is 800 times higher than that of pure g- $\mathrm{C}_{3} \mathrm{~N}_{4}$. When $\mathrm{K}_{2} \mathrm{HPO}_{4}$ was added to the photocatalytic system, the $\mathrm{H}_{2}$ production rate of the $\mathrm{Pt}_{0.5} \mathrm{Pd}_{0.5} / \mathrm{g}-\mathrm{C}_{3} \mathrm{~N}_{4}$ composite sample reached $2885.0 \mu \mathrm{mol} \cdot \mathrm{g}^{-1} \cdot \mathrm{h}^{-1}$. The AQY of $\mathrm{Pt}_{0.5} \mathrm{Pd}_{0.5} / \mathrm{g}^{-} \mathrm{C}_{3} \mathrm{~N}_{4}$ composite sample at $420 \mathrm{~nm}$ was determined to be $5.5 \%$. Therefore, our experimental results demonstrated that PtPd alloy NPs were a promising cocatalyst for g- $_{3} \mathrm{~N}_{4}$, which can also be regarded as a potential functional cocatalyst in others semiconductor photocatalysts for enhancement of the photocatalytic $\mathrm{H}_{2}$ production activities.

The high cost of noble-metals limited their application in industrial scale water splitting for $\mathrm{H}_{2}$ evolution. Hence, it is necessary to choose a suitable photocatalyst with natural
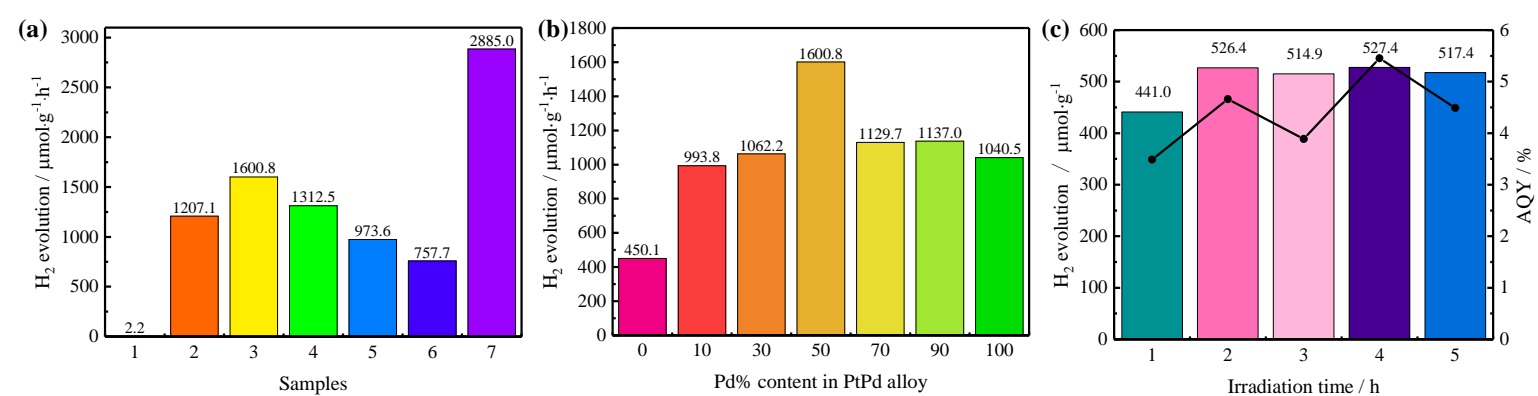

Fig. 9. (a) Photocatalytic $\mathrm{H}_{2}$ evolution over PtPd/g- $\mathrm{C}_{3} \mathrm{~N}_{4}$ composite samples with different weight contents of PtPd alloy NPs: (1) Pure g-C $\mathrm{C}_{4}$; (2) 0.1wt.\%; (3) 0.2 wt.\%; (4) 0.5 wt.\%; (5) 1.0 wt.\%; (6) 2.0 wt.\%; (7) 0.2 wt.\% with $\mathrm{K}_{2} \mathrm{HPO}_{4}$ solution. (b) Photocatalytic $\mathrm{H}_{2}$ evolution over the bulk 0.2 wt.\% $\mathrm{Pt}_{1-x} \mathrm{Pd}_{x} / \mathrm{g}-\mathrm{C}_{3} \mathrm{~N}_{4}$ composite samples with different $\mathrm{Pt} / \mathrm{Pd}$ percentage. (c) The photocatalytic $\mathrm{H}_{2}$ evolution and quantum efficiency in the presence of 0.2 wt.\% PtPd/g- $\mathrm{C}_{3} \mathrm{~N}_{4}$ composite sample under $400 \mathrm{~nm}$ light irradiation. From Ref. [127]. 
abundance cocatalyst that constructing an efficient material for water splitting. Non-noble metal elements of $\mathrm{Ni}$ or Co show the similar characteristics with noble Pt. Our group [128] reported that PtCo bimetallic alloy as cocatalyst to modified on g $^{-} \mathrm{C}_{3} \mathrm{~N}_{4}$ nanosheets via an in-situ chemical deposition method, which replace part Pt with Co to reduce the high cost. The $1.0 \mathrm{wt} . \%$ $\mathrm{PtCo} / \mathrm{g}-\mathrm{C}_{3} \mathrm{~N}_{4}$ showed the highest rate of photocatalytic $\mathrm{H}_{2}$ evolution of $960 \mu \mathrm{mol} \cdot \mathrm{g}^{-1} \cdot \mathrm{h}^{-1}$, which is 2.9 times higher than that of pristine bulk PtCo/g- $\mathrm{C}_{3} \mathrm{~N}_{4}$ under visible light irradiation. Similarly, Peng et al. [138] found that the PtNi bimetallic alloy decorated on $\mathrm{g}-\mathrm{C}_{3} \mathrm{~N}_{4}$ nanotubes could improve the photocatalytic activity, and the $\mathrm{H}_{2}$ generation rate is $104.7 \mu \mathrm{mol} \cdot \mathrm{g}^{-1} \cdot \mathrm{h}^{-1}$, which is about 47.6 and 1.1 times higher than that of pure $g-\mathrm{C}_{3} \mathrm{~N}_{4}$ nanotubes and $\mathrm{Pt} / \mathrm{g}-\mathrm{C}_{3} \mathrm{~N}_{4}$, respectively. Hence, PtCo and PtNi bimetallic alloy could replace $\mathrm{Pt}$ as a promising cocatalyst for photocatalytic $\mathrm{H}_{2}$ evolution with better activity and lower cost.

For further cost reductions, the choice of two metals in the alloy are all non-noble metals. In 2018, Zhang's group [134] reported that $\mathrm{Ni}_{0.8} \mathrm{Co}_{0.2}$ alloy as cocatalysts decorated on $\mathrm{Zn}_{0.75} \mathrm{Cd}_{0.25} \mathrm{~S}$ with optimized $\mathrm{H}_{2}$ evolution rate of 211.4 $\mathrm{mmol} \cdot \mathrm{h}^{-1} \cdot \mathrm{g}^{-1}$ and AQY of $13.3 \%$ at $365 \mathrm{~nm}$. Besides, the NiCd bimetallic nanoparticle can also be used as cocatalyst to enhance the rate of $\mathrm{H}_{2}$ evolution with dropping on $\mathrm{CdS}$ nanorods which reported by Liu and co-workers [132]. The NiCd/CdS NRs sample exhibited a palpable improved in photocatalytic $\mathrm{H}_{2}$ production performance, the optimization rate of $\mathrm{H}_{2}$ evolution was determined to be $11.57 \mathrm{mmol} \cdot \mathrm{h}^{-1} \cdot \mathrm{g}^{-1}$, which is about 64.8 , 17.2 and 2.3 times that of pristine CdS NRs, Cd/CdS and Ni/CdS, respectively.

\subsection{Metal phosphides}

Among the metal-based cocatalysts, metal phosphides are currently broadly studied in photocatalytic fields because of its good stability and high activity. Among the so many developed metal phosphides, metal phosphides composed by the elements of transition metals such as $\mathrm{Co}, \mathrm{Ni}$ and $\mathrm{Fe}$ are getting more research attention. Firstly, phosphorus is abundant in the earth, which is easy to obtain and in low cost. Secondly, compared with other metal-based materials, the transition metal phosphides are easier to synthesize. Thirdly, the P-ligand in the phosphide (namely "the ensemble effect") has a moderate binding strength to hydrogen, which is important for the formation of $\mathrm{H}_{2}$ molecules and the decrease of $\mathrm{H}$-poisoning effects $[142,143]$. Typical single transition metal phosphides including CoP [16,41], $\mathrm{Co}_{2} \mathrm{P}$ [144], $\mathrm{Ni}_{2} \mathrm{P}$ [42], $\mathrm{Ni}_{12} \mathrm{P}_{5}$ [145] $\mathrm{Cu}_{3} \mathrm{P}$ [146], FeP [147], etc:; multiple transition metal phosphides are mainly NiCoP [148]. In this section, the application of transition metal phosphide materials as cocatalysts for photocatalytic $\mathrm{H}_{2}$ production is introduced, and Table 4 summarizes the application of non-metals as cocatalysts in photocatalyst system for $\mathrm{H}_{2}$ evolution.

Han et al. [16] have synthesized $\mathrm{CoP} / \mathrm{g}-\mathrm{C}_{3} \mathrm{~N}_{4}$ photocatalysts by in-situ chemical deposition for enhanced photocatalytic hydrogen production performance. The maximum rate of hydrogen production is $9.2 \mu \mathrm{mol} \cdot \mathrm{h}^{-1}$. In recent years, our group always committed to explore the transition metal phosphides as cocatalysts for $\mathrm{H}_{2}$ evolution. Noble-metal-free photocatalyst $\mathrm{Co}_{2} \mathrm{P}-\mathrm{CdS}$ was synthesized via a facile in situ hydrothermal

Table 4

Summary of metal phosphides as cocatalysts in the photocatalyst system for $\mathrm{H}_{2}$ evolution.

\begin{tabular}{|c|c|c|c|c|c|c|c|}
\hline Photocatalyst & Cocatalyst & Light source & $\begin{array}{l}\text { Weight } \\
\text { (mg) }\end{array}$ & Solution & Activity & AQY & Year/Ref. \\
\hline 4.0 wt. $\%$ WP/CdS & WP & $300 \mathrm{~W}$ Xe lamp $(\lambda \geq 420 \mathrm{~nm})$ & 50 & $\begin{array}{c}100 \mathrm{~mL} 1.0 \mathrm{M} \\
\left(\mathrm{NH}_{4}\right)_{2} \mathrm{SO}_{3}\end{array}$ & $155.2 \mu \mathrm{mol} \cdot \mathrm{h}^{-1} \cdot \mathrm{g}^{-1}$ & - & 2017 [151] \\
\hline $\mathrm{CdS} / \mathrm{Co}_{2} \mathrm{P}$ & $\mathrm{Co}_{2} \mathrm{P}$ & 300 W Xe lamp & 50 & $\begin{array}{l}200 \mathrm{~mL} 10 \text { vol\% } \\
\text { lactic acid }\end{array}$ & $10.8 \mathrm{mmol} \cdot \mathrm{h}^{-1} \cdot \mathrm{g}^{-1}$ & - & 2016 [152] \\
\hline $1.2 \mathrm{~mol} \% \mathrm{Co}_{2} \mathrm{P} / \mathrm{CdS}$ & $\mathrm{Co}_{2} \mathrm{P}$ & $300 \mathrm{~W}$ Xe lamp $(\lambda \geq 420 \mathrm{~nm})$ & 50 & $\begin{array}{c}100 \mathrm{~mL} 0.35 \mathrm{M} \\
\mathrm{Na}_{2} \mathrm{~S} / 0.25 \mathrm{M} \mathrm{Na}_{2} \mathrm{SO}_{3}\end{array}$ & $0.303 \mathrm{mmol} \cdot \mathrm{h}^{-1}$ & $\begin{array}{c}13.88 \% \\
(420 \mathrm{~nm})\end{array}$ & 2018 [144] \\
\hline $\mathrm{CoP} / \mathrm{Zn}_{0.5} \mathrm{Cd}_{0.5} \mathrm{~S}$ & $\mathrm{CoP}$ & $300 \mathrm{~W}$ Xe lamp $(\lambda \geq 420 \mathrm{~nm})$ & 50 & $\begin{array}{c}100 \mathrm{~mL} 10 \mathrm{vol} \% \\
\text { lactic acid }\end{array}$ & $734 \mu \mathrm{mol} \cdot \mathrm{h}^{-1}$ & - & 2017 [41] \\
\hline g- $\mathrm{C}_{3} \mathrm{~N}_{4} / \mathrm{CoP}-3.4 \%$ & $\mathrm{CoP}$ & $300 \mathrm{~W}$ Xe lamp $(\lambda \geq 420 \mathrm{~nm})$ & 10 & 10 mL 10 vol\% TEOA & $0.8 \mu \mathrm{mol} \cdot 2 \mathrm{~h}^{-1} \cdot \mathrm{g}^{-1}$ & - & 2017 [153] \\
\hline 3.0 wt. $\%$ CoP $/ g-C_{3} \mathrm{~N}_{4}$ & $\mathrm{CoP}$ & 300 W Xe lamp $(\lambda \geq 420 \mathrm{~nm})$ & 50 & $\begin{array}{c}100 \mathrm{~mL} 10 \text { vol\% TEOA } \\
\text { and } \mathrm{K}_{2} \mathrm{HPO}_{4}\end{array}$ & $15.1 \mu \mathrm{mol} \cdot \mathrm{h}^{-1}$ & - & 2019 [16] \\
\hline $4 \% \mathrm{Ni}_{2} \mathrm{P} / \mathrm{Zn}_{0.5} \mathrm{Cd}_{0.5} \mathrm{~S}$ & $\mathrm{Ni}_{2} \mathrm{P}$ & $300 \mathrm{~W}$ Xe lamp $(\lambda \geq 420 \mathrm{~nm})$ & 50 & $\begin{array}{c}100 \mathrm{~mL} 0.35 \mathrm{M} \\
\mathrm{Na}_{2} \mathrm{~S} / 0.25 \mathrm{M} \mathrm{Na}_{2} \mathrm{SO}_{3}\end{array}$ & $1173 \mu \mathrm{mol} \cdot \mathrm{h}^{-1}$ & - & 2018 [42] \\
\hline g- $\mathrm{C}_{3} \mathrm{~N}_{4} / \mathrm{Ni}_{2} \mathrm{P}-3.5$ wt. $\%$ & $\mathrm{Ni}_{2} \mathrm{P}$ & $\begin{array}{c}300 \mathrm{~W} \text { high-pressure Xe } \\
\operatorname{lamp}(\lambda \geq 420 \mathrm{~nm})\end{array}$ & 50 & $100 \mathrm{~mL} 10$ vol\% TEOA & $474.7 \mu \mathrm{mol} \cdot \mathrm{h}^{-1} \cdot \mathrm{g}^{-1}$ & $3.2 \%(435 \mathrm{~nm})$ & 2018 [154] \\
\hline g- $\mathrm{C}_{3} \mathrm{~N}_{4} / \mathrm{Ni}_{2} \mathrm{P}-0.48$ wt. $\%$ & $\mathrm{Ni}_{2} \mathrm{P}$ & $300 \mathrm{~W}$ Xe lamp $(\lambda \geq 420 \mathrm{~nm})$ & 10 & $10 \mathrm{~mL} 10$ vol\% TEOA & $5.67 \mu \mathrm{mol} \cdot \mathrm{h}^{-1}$ & - & 2017 [155] \\
\hline $\begin{array}{l}5 \text { wt. } \% \mathrm{Ni}_{2} \mathrm{P} / \\
0.5-\mathrm{Fe}^{3+}-\mathrm{g}-\mathrm{C}_{3} \mathrm{~N}_{4}\end{array}$ & $\mathrm{Ni}_{2} \mathrm{P}$ & $300 \mathrm{~W}$ Xe lamp $(\lambda \geq 420 \mathrm{~nm})$ & 100 & $100 \mathrm{~mL} 10$ vol\% TEOA & $1891 \mu \mathrm{mol} \cdot \mathrm{h}^{-1} \cdot \mathrm{g}^{-1}$ & - & 2017 [156] \\
\hline $\mathrm{Ni}_{2} \mathrm{P} / \mathrm{g}-\mathrm{C}_{3} \mathrm{~N}_{4}$ & $\mathrm{Ni}_{2} \mathrm{P}$ & Xe lamp $(\lambda \geq 420 \mathrm{~nm})$ & 2 & $5 \mathrm{~mL} 20$ vol\% TEOA & $162 \mu \mathrm{mol} \cdot \mathrm{h}^{-1} \cdot \mathrm{g}^{-1}$ & - & 2017 [149] \\
\hline 0.4 wt. $\% \mathrm{Ni}_{2} \mathrm{P} / \mathrm{TiO}_{2}$ & $\mathrm{Ni}_{2} \mathrm{P}$ & $300 \mathrm{~W}$ Xe lamp $(\lambda \geq 400 \mathrm{~nm})$ & 20 & $\begin{array}{l}80 \mathrm{~mL} 50 \text { vol\% } \\
\text { methanol }\end{array}$ & $9.38 \mathrm{mmol} \cdot \mathrm{h}^{-1} \cdot \mathrm{g}^{-1}$ & $\begin{array}{c}11.6 \% \\
(360 \mathrm{~nm})\end{array}$ & 2016 [157] \\
\hline 5 wt. $\% \mathrm{Ni}_{12} \mathrm{P}_{5} / \mathrm{g}-\mathrm{C}_{3} \mathrm{~N}_{4}$ & $\mathrm{Ni}_{12} \mathrm{P}_{5}$ & $\begin{array}{l}300 \mathrm{~W} \text { high-pressure Xe } \\
\text { lamp }(\lambda \geq 420 \mathrm{~nm})\end{array}$ & 60 & $150 \mathrm{~mL} 10$ vol\% TEOA & $535.7 \mu \mathrm{mol} \cdot \mathrm{h}^{-1} \cdot \mathrm{g}^{-1}$ & $\begin{array}{c}4.67 \% \\
(420 \mathrm{~nm})\end{array}$ & 2017 [145] \\
\hline 5 wt. $\% \mathrm{FeP} / \mathrm{CdS}$ & $\mathrm{FeP}$ & $30 \times 3 \mathrm{~W} \operatorname{LED}(\lambda \geq 420 \mathrm{~nm})$ & 1 & $\begin{array}{l}10 \mathrm{~mL} 10 \mathrm{vol} \% \\
\text { lactic acid }\end{array}$ & $202000 \mu \mathrm{mol} \cdot 5 \mathrm{~h}^{-1} \cdot \mathrm{g}^{-1}$ & $\begin{array}{c}35 \% \\
(520 \mathrm{~nm})\end{array}$ & 2016 [147] \\
\hline $\mathrm{g}-\mathrm{C}_{3} \mathrm{~N}_{4} / \mathrm{Fe}_{x} \mathrm{P}-0.08$ & $\mathrm{Fe}_{\mathrm{x}} \mathrm{P}$ & $300 \mathrm{~W}$ Xe lamp $(\lambda \geq 420 \mathrm{~nm})$ & 10 & $10 \mathrm{~mL} 10$ vol$\%$ TEOA & $166.4 \mu \mathrm{mol} \cdot 2 \mathrm{~h}^{-1} \cdot \mathrm{g}^{-1}$ & - & 2017 [150] \\
\hline 10 wt. $\% \mathrm{Cu}_{3} \mathrm{P} / \mathrm{g}-\mathrm{C}_{3} \mathrm{~N}_{4}$ & $\mathrm{Cu}_{3} \mathrm{P}$ & $300 \mathrm{~W}$ Xe lamp $(\lambda \geq 420 \mathrm{~nm})$ & 50 & $80 \mathrm{~mL} 15$ vol\% TEOA & $159.41 \mu \mathrm{mol} \cdot \mathrm{h}^{-1} \cdot \mathrm{g}^{-1}$ & - & 2018 [146] \\
\hline $\mathrm{NiCoP} / \mathrm{g}-\mathrm{C}_{3} \mathrm{~N}_{4}$ & $\mathrm{NiCoP}$ & $300 \mathrm{~W}$ Xe lamp $(\lambda \geq 420 \mathrm{~nm})$ & 50 & $80 \mathrm{~mL} 10$ vol\% TEOA & $1709.3 \mu \mathrm{mol} \cdot \mathrm{h}^{-1} \cdot \mathrm{g}^{-1}$ & $9.4 \%(420 \mathrm{~nm})$ & 2017 [148] \\
\hline
\end{tabular}


(a)

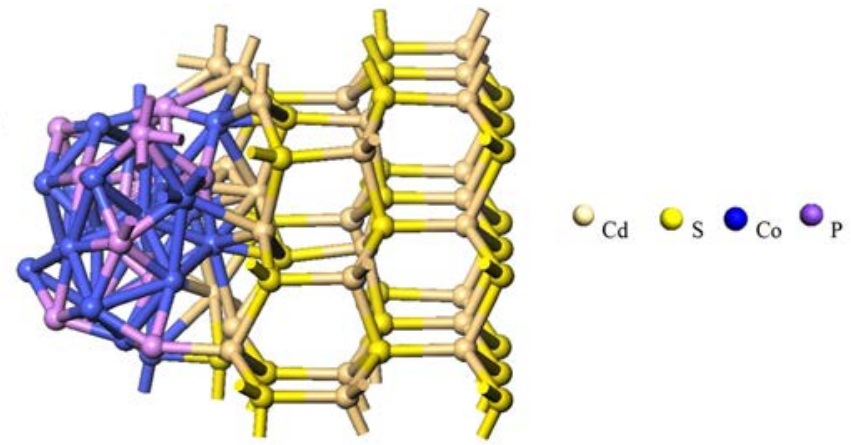

(b)
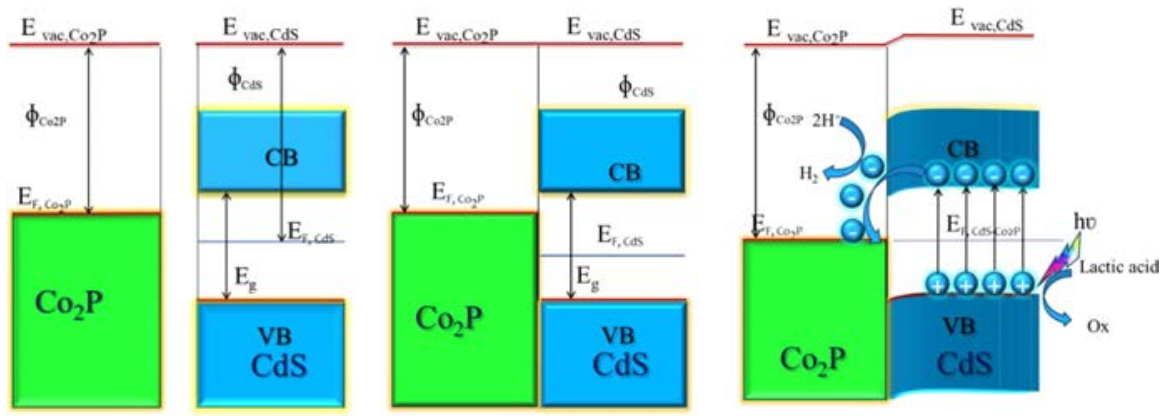

Fig. 10. Schematic of photocatalysis by $\mathrm{Co}_{2} \mathrm{P}-\mathrm{CdS}$ composites. (a) Illustration of combination between metal Co $2 \mathrm{P}(\mathrm{Co}$, blue; $\mathrm{P}$, purple) and semiconductor CdS (S, yellow; Cd, dull yellow). (b) Calculated work function and band bending of metal $\mathrm{Co}_{2} \mathrm{P}$ and CdS. From Ref. [144].

method [144]. The $\mathrm{Co}_{2} \mathrm{P}-\mathrm{CdS}$ sample with a loading ratio of 1.2 mol\% $\mathrm{Co}_{2} \mathrm{P}$ gave the highest $\mathrm{H}_{2}$ production rate of 0.303 $\mathrm{mmol} \cdot \mathrm{h}^{-1}$, after further introduction of $\mathrm{K}_{2} \mathrm{HPO}_{4}$ as sacrificial agent, the $\mathrm{H}_{2}$ production rate reached $0.356 \mathrm{mmol} \cdot \mathrm{h}^{-1}$, and the $\mathrm{AQE}$ of the $\mathrm{Co}_{2} \mathrm{P}$-decorated CdS sample is about $13.88 \%$ at 420 $\mathrm{nm}$. The application of $\mathrm{Co}_{2} \mathrm{P}$ as co-catalyst has significantly improved the photocatalytic $\mathrm{H}_{2}$ evolution performance of CdS. When $\mathrm{CdS}$ and $\mathrm{Co}_{2} \mathrm{P}$ form intimate contact, the electrons transfer from $\mathrm{Co}_{2} \mathrm{P}$ to $\mathrm{CdS}$, which leads to a downward bending of the energy bands of CdS, the separation and transfer of photo charges can be boosted as a result of the band bending formed at the interface, which leads to a dramatically enhanced performance of photocatalytic $\mathrm{H}_{2}$ production (Fig. 10).

Sun et al. [149] tested the performance of three different crystalline phases of $\mathrm{Ni}_{2} \mathrm{P}, \mathrm{Ni}_{12} \mathrm{P}_{5}$, and $\mathrm{Ni}_{3} \mathrm{P}$ as cocatalysts to hybridize with g- $\mathrm{C}_{3} \mathrm{~N}_{4}$ for photocatalytic $\mathrm{H}_{2}$ generation in 2017. As shown in Fig. 11, all three phases of $\mathrm{Ni}_{x} \mathrm{P}_{y}$ work as eximious cocatalysts for enhancement of visible light $\mathrm{H}_{2}$ generation with g- $\mathrm{C}_{3} \mathrm{~N}_{4}$. The highest $\mathrm{H}_{2}$ generation activities by achieved with 5 wt.\% $\mathrm{Ni}_{2} \mathrm{P} / \mathrm{g}-\mathrm{C}_{3} \mathrm{~N}_{4}$ was $0.162 \mathrm{mmol} \cdot \mathrm{h}^{-1} \cdot \mathrm{g}^{-1}$, which was approximately 34 times of the amount obtained on pure g- $\mathrm{C}_{3} \mathrm{~N}_{4}$, and the rate was close to $5 \mathrm{wt} . \% \mathrm{Pt} / \mathrm{g}-\mathrm{C}_{3} \mathrm{~N}_{4}\left(0.168 \mathrm{mmol} \cdot \mathrm{h}^{-1} \cdot \mathrm{g}^{-1}\right)$. In addition, the photocatalyst exhibited favorable stability after 9 hours illumination under visible light irradiation.

Dong's group [150] reported that noble-metal-free iron phosphide $\left(\mathrm{Fe}_{x} \mathrm{P}\right)$ cocatalyst decorated graphitic carbon nitride ( $\mathrm{g}-\mathrm{C}_{3} \mathrm{~N}_{4}$ ) as photocatalysts by two-step hydrothermal and phosphidation method for the highly efficient and stable $\mathrm{H}_{2}$ evolution from water splitting under visible light irradiated. The $\mathrm{g}_{3} \mathrm{C}_{3} \mathrm{~N}_{4} / \mathrm{Fe}_{x} \mathrm{P}-0.08$ showed the optimal rate of photocatalytic $\mathrm{H}_{2}$ generation, the rate reached up to $166.4 \mu \mathrm{mol} \cdot \mathrm{h}^{-1} \cdot \mathrm{g}^{-1}$, which was about 277 and 139 times that over pristine g- $\mathrm{C}_{3} \mathrm{~N}_{4}$ and $\mathrm{g}-\mathrm{C}_{3} \mathrm{~N}_{4} / \mathrm{FeOOH}$ precursor, respectively. The $\mathrm{Fe}_{x} \mathrm{P}$ as cocatalyst significantly accelerates the separation and transfer of photogenerated charges which lead to the highly efficient photocatalytic $\mathrm{H}_{2}$ evolution activity of g- $\mathrm{C}_{3} \mathrm{~N}_{4}$.

In 2017, Qin et al. [148] synthetized NiCoP core/shell NPs as cocatalyst by a solid-state phosphorization reaction. NiCoP@NiCo-Pi/g- $\mathrm{C}_{3} \mathrm{~N}_{4}$ exhibited much higher hydrogen production rate than $\mathrm{Ni}_{2} \mathrm{P} @ \mathrm{NiCo}-\mathrm{Pi} / \mathrm{g}-\mathrm{C}_{3} \mathrm{~N}_{4}$ and CoP@NiCo-Pi/g- $\mathrm{C}_{3} \mathrm{~N}_{4}$, which demonstrated better performance of NiCoP cocatalysts for photocatalytic $\mathrm{H}_{2}$ evolution than the single transition metal phosphides.

\subsection{Metal oxides and hydroxides}

Several metal oxides such as $\mathrm{TiO}_{2}$ [158-161], $\mathrm{CuO}$ [22,162-165], $\mathrm{Cu}_{2} \mathrm{O}[49,166,167]$ and $\mathrm{NiO}[168]$ are well known as cocatalysts for photocatalytic $\mathrm{H}_{2}$ production. Table 5 summarizes the application of metal oxides and hydroxides as cocatalyst in the photocatalyst system for $\mathrm{H}_{2}$ evolution. Recently, Tan et al. [160] reported the nanostructured
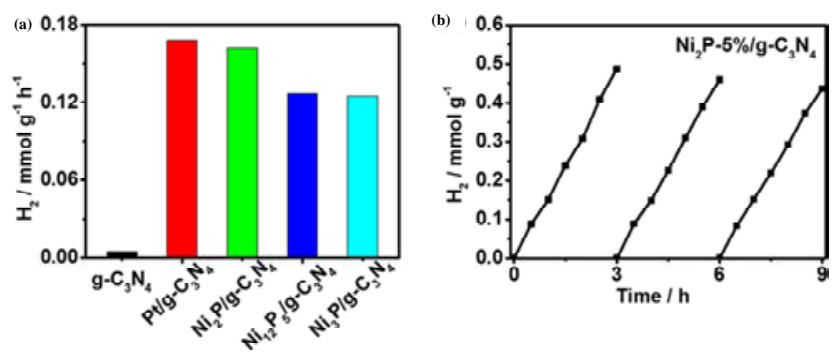

Fig. 11. (a) Photocatalytic $\mathrm{H}_{2}$ generation rates for g- $\mathrm{C}_{3} \mathrm{~N}_{4}, \mathrm{Pt} / \mathrm{g}-\mathrm{C}_{3} \mathrm{~N}_{4}$, and $\mathrm{Ni}_{x} \mathrm{P}_{y} / g-\mathrm{C}_{3} \mathrm{~N}_{4}$ (5 wt.\% $\mathrm{Ni}_{x} \mathrm{P}_{y}$ ). (b) Recycle of $\mathrm{H}_{2}$ generation on $\mathrm{Ni}_{2} \mathrm{P} / \mathrm{g}-\mathrm{C}_{3} \mathrm{~N}_{4}$ (5 wt.\% $\mathrm{Ni}_{x} \mathrm{P}_{y}$ ). From Ref. [149]. 
Table 5

Summary of metal oxides and hydroxides as cocatalysts in the photocatalyst systems for $\mathrm{H}_{2}$ evolution.

\begin{tabular}{|c|c|c|c|c|c|c|c|}
\hline Photocatalyst & Cocatalyst & Light source & $\begin{array}{l}\text { Weight } \\
\text { (mg) }\end{array}$ & Solution & Activity & AQY & Year/Ref. \\
\hline $\mathrm{TiO}_{2} / \mathrm{g}-\mathrm{C}_{3} \mathrm{~N}_{4}$ & $\mathrm{TiO}_{2}$ & $\begin{array}{c}300 \mathrm{~W} \text { Xe lamp } \\
(\lambda \geq 420 \mathrm{~nm})\end{array}$ & 100 & $\begin{array}{c}100 \mathrm{~mL} 10 \text { vol } \% \\
\text { TEOA }\end{array}$ & $513 \mu \mathrm{mol} \cdot \mathrm{h}^{-1} \cdot \mathrm{g}^{-1}$ & $0.31 \%$ & 2018 [160] \\
\hline $\mathrm{B}-\mathrm{TiO}_{2} / \mathrm{g}-\mathrm{C}_{3} \mathrm{~N}_{4}$ & $\mathrm{~B}-\mathrm{TiO}_{2}$ & $\begin{array}{l}300 \mathrm{~W} \text { Xe lamp } \\
(\lambda \geq 420 \mathrm{~nm})\end{array}$ & 50 & $\begin{array}{l}100 \mathrm{~mL} 10 \text { vol } \% \\
\text { TEOA }\end{array}$ & $808.97 \mu \mathrm{mol} \cdot \mathrm{h}^{-1} \cdot \mathrm{g}^{-1}$ & - & 2019 [161] \\
\hline $0.330 \% \mathrm{C}-\mathrm{TiO}_{2} / \mathrm{g}-\mathrm{C}_{3} \mathrm{~N}_{4}$ & $\mathrm{C}-\mathrm{TiO}_{2}$ & $\begin{array}{c}300 \text { W Xe lamp } \\
(\lambda \geq 420 \mathrm{~nm})\end{array}$ & 50 & $\begin{array}{c}80 \mathrm{~mL} 10 \text { vol\% } \\
\text { TEOA }\end{array}$ & $\begin{array}{c}5.728 \mathrm{mmol} \cdot 5 \mathrm{~h}^{-1} \cdot \mathrm{g}^{-1} \\
52.395 \mathrm{mmol} \cdot 64 \mathrm{~h}^{-1} \cdot \mathrm{g}^{-1}\end{array}$ & $\begin{array}{c}6.2 \% \\
(420 \mathrm{~nm})\end{array}$ & 2017 [159] \\
\hline 2.0 wt. $\% \mathrm{TiO}_{2} / \mathrm{g}-\mathrm{C}_{3} \mathrm{~N}_{4}$ & $\mathrm{TiO}_{2}$ & $\begin{array}{l}3 \mathrm{~W} \text { UV-LEDs } \\
(\lambda \geq 420 \mathrm{~nm})\end{array}$ & 50 & $\begin{array}{l}80 \mathrm{~mL} 25 \text { vol } \% \\
\text { methanol }\end{array}$ & $5.6 \mu \mathrm{mol} \cdot \mathrm{h}^{-1}$ & $\begin{array}{c}3.25 \% \\
(420 \mathrm{~nm})\end{array}$ & 2016 [158] \\
\hline $0.4 \% \mathrm{Bi}_{2} \mathrm{O}_{3} / \mathrm{TiO}_{2}$ & $\mathrm{Bi}_{2} \mathrm{O}_{3}$ & natural solar light & 5 & $\begin{array}{l}50 \mathrm{~mL} 5 \text { vol } \% \\
\text { glycerol }\end{array}$ & $26.02 \mathrm{mmol} \cdot \mathrm{h}^{-1} \cdot \mathrm{g}^{-1}$ & - & 2017 [179] \\
\hline $\begin{array}{l}0.83 \text { wt. } \% \\
\mathrm{Ag}_{2} \mathrm{O} / \mathrm{g}-\mathrm{C}_{3} \mathrm{~N}_{4}\end{array}$ & $\mathrm{Ag}_{2} \mathrm{O}$ & $\begin{array}{c}300 \mathrm{~W} \text { Xe lamp } \\
(\lambda \geq 420 \mathrm{~nm})\end{array}$ & 100 & $\begin{array}{l}100 \mathrm{~mL} 10 \text { vol } \% \\
\text { TEOA }\end{array}$ & $33.04 \mu \mathrm{mol} \cdot \mathrm{h}^{-1}$ & - & 2015 [170] \\
\hline $2.5 \mathrm{~mol} \% \mathrm{Cu}_{2} \mathrm{O} / \mathrm{TiO}_{2}$ & $\mathrm{Cu}_{2} \mathrm{O}$ & $\begin{array}{c}500 \mathrm{~W} \text { Xe lamp } \\
(\lambda \geq 420 \mathrm{~nm})\end{array}$ & $\begin{array}{l}\text { a certain } \\
\text { amount }\end{array}$ & $\begin{array}{l}450 \mathrm{~mL} 10 \text { vol\% } \\
\text { methanol }\end{array}$ & $2048.25 \mu \mathrm{mol} \cdot \mathrm{h}^{-1} \cdot \mathrm{g}^{-1}$ & $\begin{array}{c}4.32 \% \\
(365 \mathrm{~nm})\end{array}$ & 2015 [167] \\
\hline $10 \% \mathrm{CuO} / \mathrm{TiO}_{2}$ & $\mathrm{CuO}$ & 500 W Xe lamp & 100 & $\begin{array}{l}100 \mathrm{~mL} 25 \text { vol\% } \\
\text { methanol }\end{array}$ & $8.23 \mathrm{mmol} \cdot \mathrm{h}^{-1} \cdot \mathrm{g}^{-1}$ & - & $2013[22]$ \\
\hline $0.9 \mathrm{~mol} \% \mathrm{Cu}_{2} \mathrm{O} / \mathrm{TiO}_{2}$ & $\mathrm{Cu}_{2} \mathrm{O}$ & $\begin{array}{c}300 \mathrm{~W} \text { Xe lamp } \\
(\lambda \geq 320 \mathrm{~nm})\end{array}$ & 50 & $\begin{array}{l}40 \mathrm{~mL} 0.05 \mathrm{M} \\
\text { glycerol }\end{array}$ & $318 \mu \mathrm{mol} \cdot \mathrm{h}^{-1}$ & $\begin{array}{c}28.6 \% \\
(365 \mathrm{~nm})\end{array}$ & 2013 [166] \\
\hline $\begin{array}{l}0.05 \text { wt. } \% \\
\mathrm{Cu}_{2} \mathrm{O} / \mathrm{g}-\mathrm{C}_{3} \mathrm{~N}_{4}\end{array}$ & $\mathrm{Cu}_{2} \mathrm{O}$ & $\begin{array}{l}300 \text { W Xe lamp } \\
(\lambda \geq 420 \mathrm{~nm})\end{array}$ & 100 & $\begin{array}{l}180 \mathrm{~mL} 10 \text { vol } \% \\
\text { TEOA }\end{array}$ & $241.3 \mu \mathrm{mol} \cdot \mathrm{h}^{-1} \cdot \mathrm{g}^{-1}$ & - & 2014 [49] \\
\hline 0.25 wt. $\% \mathrm{NiO} / \mathrm{TiO}_{2}$ & $\mathrm{NiO}$ & 300 W Xe lamp & 50 & $\begin{array}{l}80 \mathrm{~mL} 25 \text { vol\% } \\
\text { methanol }\end{array}$ & $337 \mu \mathrm{mol} \cdot \mathrm{h}^{-1} \cdot \mathrm{g}^{-1}$ & $\begin{array}{c}1.7 \% \\
(365 \mathrm{~nm})\end{array}$ & 2015 [168] \\
\hline 1.3 wt. $\% \mathrm{CuO} / \mathrm{TiO}_{2}$ & $\mathrm{CuO}$ & $\begin{array}{c}3 \mathrm{~W} \text { UV-LEDs } \\
(\lambda=365 \mathrm{~nm})\end{array}$ & 80 & $\begin{array}{l}80 \mathrm{~mL} 0.1 \mathrm{M} \\
\text { glycerol }\end{array}$ & $2061 \mu \mathrm{mol} \cdot \mathrm{h}^{-1} \cdot \mathrm{g}^{-1}$ & $\begin{array}{c}13.4 \% \\
(365 \mathrm{~nm})\end{array}$ & 2011 [162] \\
\hline $23 \mathrm{~mol} \% \mathrm{Ni}(\mathrm{OH})_{2} / \mathrm{CdS}$ & $\mathrm{Ni}(\mathrm{OH})_{2}$ & $\begin{array}{c}300 \text { W Xe lamp } \\
(\lambda \geq 420 \mathrm{~nm})\end{array}$ & 50 & $\begin{array}{c}80 \mathrm{~mL} 25 \text { vol } \% \\
\text { TEOA }\end{array}$ & $5084 \mu \mathrm{mol} \cdot \mathrm{h}^{-1} \cdot \mathrm{g}^{-1}$ & $\begin{array}{c}28 \% \\
(420 \mathrm{~nm})\end{array}$ & $2011[171]$ \\
\hline $\begin{array}{l}0.23 \mathrm{~mol} \% \\
\mathrm{Ni}(\mathrm{OH})_{2} / \mathrm{TiO}_{2}\end{array}$ & $\mathrm{Ni}(\mathrm{OH})_{2}$ & $\begin{array}{l}3 \mathrm{~W} \text { UV-LEDs } \\
(\lambda=365 \mathrm{~nm})\end{array}$ & 50 & $\begin{array}{l}80 \mathrm{~mL} 25 \text { vol\% } \\
\text { methanol }\end{array}$ & $3056 \mu \mathrm{mol} \cdot \mathrm{h}^{-1} \cdot \mathrm{g}^{-1}$ & $\begin{array}{c}12.4 \% \\
(365 \mathrm{~nm})\end{array}$ & 2011 [19] \\
\hline $\begin{array}{l}0.5 \mathrm{~mol}^{2} \% \\
\mathrm{Ni}(\mathrm{OH})_{2} / \mathrm{g}-\mathrm{C}_{3} \mathrm{~N}_{4}\end{array}$ & $\mathrm{Ni}(\mathrm{OH})_{2}$ & $\begin{array}{c}350 \text { W Xe lamp } \\
(\lambda \geq 400 \mathrm{~nm})\end{array}$ & 50 & $\begin{array}{c}80 \mathrm{~mL} 10 \text { vol } \% \\
\text { TEOA }\end{array}$ & $7.6 \mu \mathrm{mol} \cdot \mathrm{h}^{-1}$ & $\begin{array}{c}1.1 \% \\
(420 \mathrm{~nm})\end{array}$ & $2013[46]$ \\
\hline $\mathrm{Ni}(\mathrm{OH})_{2} / \mathrm{CdS}$ & $\mathrm{Ni}(\mathrm{OH})_{2}$ & $\begin{array}{c}300 \mathrm{~W} \text { Xe lamp } \\
(\lambda \geq 420 \mathrm{~nm})\end{array}$ & 100 & $\begin{array}{l}50 \mathrm{~mL} 1 \mathrm{M} \\
\left(\mathrm{NH}_{4}\right)_{2} \mathrm{SO}_{3}\end{array}$ & $40.18 \mathrm{mmol} \cdot \mathrm{h}^{-1} \cdot \mathrm{g}^{-1}$ & $\begin{array}{c}66.1 \% \\
(420 \mathrm{~nm})\end{array}$ & 2019 [180] \\
\hline $\begin{array}{l}0.34 \mathrm{~mol} \% \\
\mathrm{Cu}(\mathrm{OH})_{2} / \mathrm{g}-\mathrm{C}_{3} \mathrm{~N}_{4}\end{array}$ & $\mathrm{Cu}(\mathrm{OH})_{2}$ & $\begin{array}{c}300 \mathrm{~W} \text { Xe lamp } \\
(\lambda \geq 400 \mathrm{~nm})\end{array}$ & 100 & $\begin{array}{l}80 \mathrm{~mL} 25 \text { vol } \% \\
\text { methanol }\end{array}$ & $48.7 \mu \mathrm{mol} \cdot \mathrm{h}^{-1} \cdot \mathrm{g}^{-1}$ & - & 2014 [181] \\
\hline $\begin{array}{l}0.29 \mathrm{~mol} \% \\
\mathrm{Cu}(\mathrm{OH})_{2} / \mathrm{TiO}_{2}\end{array}$ & $\mathrm{Cu}(\mathrm{OH})_{2}$ & $\begin{array}{l}3 \mathrm{~W} \text { UV-LEDs } \\
(\lambda=365 \mathrm{~nm})\end{array}$ & 50 & $\begin{array}{l}80 \mathrm{~mL} 0.09 \mathrm{M} \\
\text { ethylene glycol }\end{array}$ & $3418 \mu \mathrm{mol} \cdot \mathrm{h}^{-1} \cdot \mathrm{g}^{-1}$ & $\begin{array}{c}13.9 \% \\
(365 \mathrm{~nm})\end{array}$ & 2011 [182] \\
\hline $\begin{array}{l}0.5 \mathrm{~mol} \% \\
\mathrm{Co}(\mathrm{OH})_{2} / \mathrm{TiO}_{2}\end{array}$ & $\mathrm{Co}(\mathrm{OH})_{2}$ & $\begin{array}{c}300 \text { W Xe lamp } \\
\text { (UV-visible light) }\end{array}$ & 50 & $\begin{array}{l}100 \mathrm{~mL} 25 \text { vol } \% \\
\text { methanol }\end{array}$ & $746.93 \mu \mathrm{mol} \cdot \mathrm{h}^{-1} \cdot \mathrm{g}^{-1}$ & - & 2017 [169] \\
\hline $\mathrm{Zn}-\mathrm{Cr}-\mathrm{LDH} / \mathrm{g}-\mathrm{C}_{3} \mathrm{~N}_{4}$ & $\mathrm{Zn}-\mathrm{Cr}-\mathrm{LDH}$ & $\begin{array}{l}300 \text { W Xe lamp } \\
(\lambda \geq 420 \mathrm{~nm})\end{array}$ & - & 10 vol $\%$ TEOA & $155.7 \mu \mathrm{mol} \cdot \mathrm{h}^{-1} \cdot \mathrm{g}^{-1}$ & - & 2018 [178] \\
\hline CdSe/Zn-Cr-LDH & $\mathrm{Zn}-\mathrm{Cr}-\mathrm{LDH}$ & $\begin{array}{l}300 \mathrm{~W} \text { Xe lamp } \\
(\lambda \geq 420 \mathrm{~nm})\end{array}$ & 50 & $\begin{array}{c}100 \mathrm{~mL} 0.1 \mathrm{M} \\
\mathrm{Na}_{2} \mathrm{~S} / 0.1 \mathrm{M} \mathrm{Na}_{2} \mathrm{SO}_{3}\end{array}$ & $2196 \mu \mathrm{mol} \cdot \mathrm{h}^{-1} \cdot \mathrm{g}^{-1}$ & $\begin{array}{c}51.3 \% \\
(420 \mathrm{~nm})\end{array}$ & 2015 [177] \\
\hline $\begin{array}{l}2 \text { mol\% Ni-Co-LDH } / 40 \\
\text { wt.\% P-CdS }\end{array}$ & $\mathrm{Ni}-\mathrm{Co}-\mathrm{LDH}$ & $\begin{array}{c}300 \mathrm{~W} \text { Xe lamp } \\
(\lambda \geq 420 \mathrm{~nm})\end{array}$ & 50 & $\begin{array}{l}100 \mathrm{~mL} 10 \text { vol\% } \\
\text { lactic acid }\end{array}$ & $8.665 \mathrm{~mol} \cdot \mathrm{h}^{-1} \cdot \mathrm{g}^{-1}$ & $\begin{array}{c}14.0 \% \\
(420 \mathrm{~nm})\end{array}$ & 2019 [183] \\
\hline
\end{tabular}

g- $\mathrm{C}_{3} \mathrm{~N}_{4} / \mathrm{TiO}_{2}$ was synthesized by controlling the growth of nanoscale $\mathrm{g}-\mathrm{C}_{3} \mathrm{~N}_{4}$ on $\mathrm{TiO}_{2}$ NPs via one step vapor deposition method. The $\mathrm{CN} / \mathrm{TiO}_{2}-24$ composite shows the greatest $\mathrm{H}_{2}$ evolution rate of $513 \mu \mathrm{mol} \cdot \mathrm{g}^{-1} \cdot \mathrm{h}^{-1}$. Under visible light irradiation, the visible-light-excited electrons in the $\mathrm{CB}$ of $\mathrm{g}-\mathrm{C}_{3} \mathrm{~N}_{4}$ can transfer to the $\mathrm{CB}$ of $\mathrm{TiO}_{2}$, the photoinduced charge could be efficiently separated, and therefore improve the photocatalytic $\mathrm{H}_{2}$ evolution of the $\mathrm{CN} / \mathrm{TiO}_{2}-24$ composite.

For transition metal oxides, $\mathrm{CuO}$ and $\mathrm{Cu}_{2} \mathrm{O}$ have also been proven to be beneficial for photocatalytic $\mathrm{H}_{2}$ production. Wang and co-worker [167] reported that $\mathrm{Cu}_{2} \mathrm{O}$ loading on $\mathrm{TiO}_{2}$ displayed 14.48 times larger photocatalytic $\mathrm{H}_{2}$ evolution activity than pure P25. Yu's group [162] found CuO can act as an effective cocatalyst to improve the photocatalytic $\mathrm{H}_{2}$ production activity of $\mathrm{TiO}_{2}$. When the loading content of $\mathrm{CuO}$ in the photocatalyst was increased to $1.3 \mathrm{wt} . \%$, the rate of $\mathrm{H}_{2}$ evolution reached $2061 \mu \mathrm{mol} \cdot \mathrm{h}^{-1} \cdot \mathrm{g}^{-1}$ with an AQY of $13.4 \%$ at $420 \mathrm{~nm}$. The quantum size effect of $\mathrm{CuO}$ influences the electron transfer and its valence band edges, which enhance the photocatalytic activity. As shown in Fig. 12(a, b), the bulk $\mathrm{CuO}$ has a less negative potential than $\mathrm{H}^{+} / \mathrm{H}_{2}$ potential. As the quantum size of $\mathrm{CuO}$ increases, the band gap decreases and the $\mathrm{CB}$ and VB edges are also shifted to the positions which permit transfer of photogenerated electrons, facilitating the interfacial electron transfer from $\mathrm{CuO}$ to $\mathrm{H}^{+}$in the solution for photocatalytic $\mathrm{H}_{2}$ evolution.

In $2015, \mathrm{Wu}$ et al. [170] synthesized $\mathrm{Ag}_{2} \mathrm{O} / \mathrm{g}-\mathrm{C}_{3} \mathrm{~N}_{4}$ via facial hydrothermal reaction, the composite photocatalyst shows efficient properties for hydrogen evolution from water splitting 

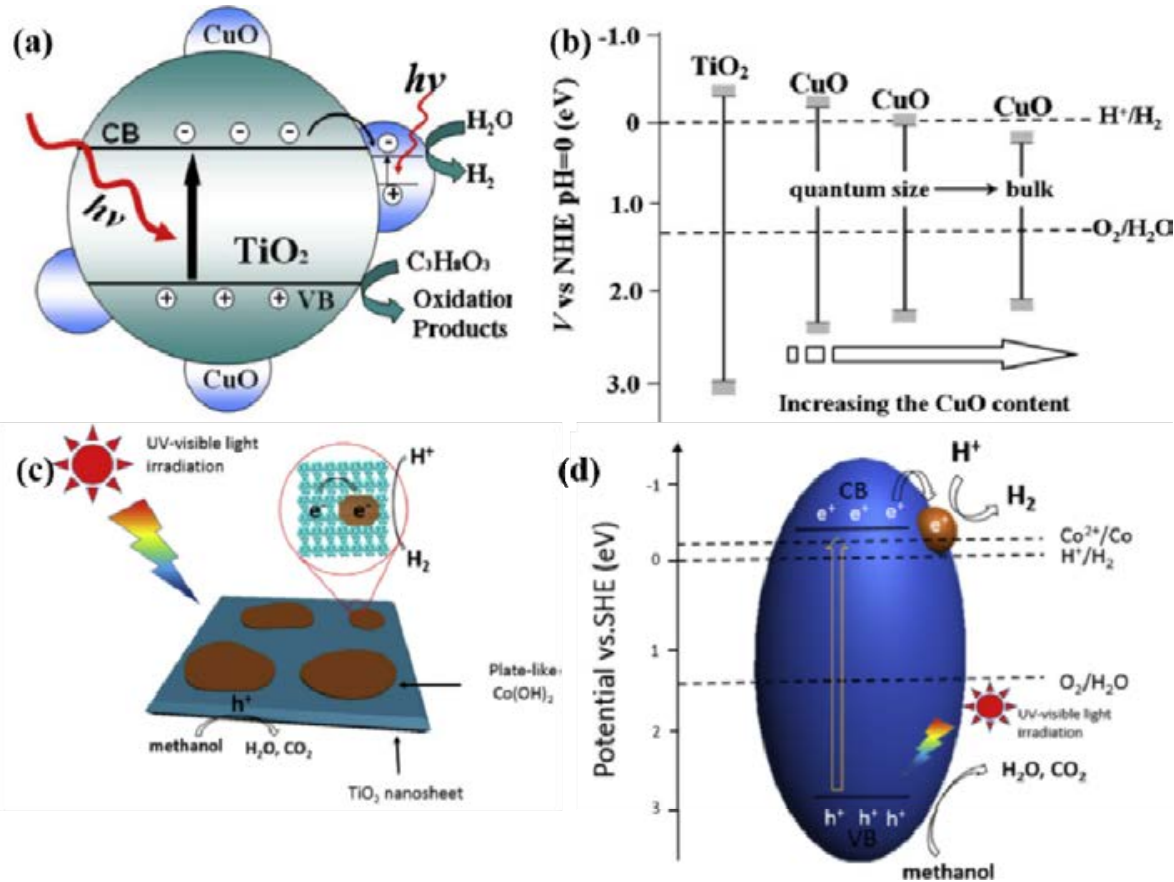

Fig. 12. (a) Schematic illustration for the charge transfer and separation in $\mathrm{CuO}_{-} \mathrm{TiO}_{2}$ under UV light. (b) Energy levels of the conduction and valence band edges vs normal hydrogen electrode (NHE, at $\mathrm{pH}$ 0) for $\mathrm{TiO}_{2}$ and $\mathrm{CuO}$ with various size (the quantum size effect is reflected by the band gap increase). From Ref. [162]. (c) Schematic illustration for the charge transfer and separation in plate-like $\mathrm{Co}(\mathrm{OH})_{2} / \mathrm{TiO}_{2}$ nanosheets system; (d) Proposed mechanism for photocatalytic $\mathrm{H}_{2}$ production under UV-Visible light irradiation. From Ref. [169].

under visible light irradiation, in which the rate of hydrogen evolution is $33.04 \mu \mathrm{mol} \cdot \mathrm{h}^{-1}$. For the mechanism, the $\mathrm{Ag}_{2} \mathrm{O}$ as a cocatalyst plays an important role in charges transferred of photocatalytic progress. The reason relies on the relative position of $\mathrm{CB}$ and $\mathrm{VB}$ between $\mathrm{g}-\mathrm{C}_{3} \mathrm{~N}_{4}$ and $\mathrm{Ag}_{2} \mathrm{O}$, in which the $\mathrm{CB}$ of $\mathrm{Ag}_{2} \mathrm{O}$ is lower than the redox potential of $\mathrm{H}^{+} / \mathrm{H}_{2}$, so that the photogenerated electrons of $\mathrm{g}-\mathrm{C}_{3} \mathrm{~N}_{4}$ cannot be transferred to the $\mathrm{CB}$ of $\mathrm{Ag}_{2} \mathrm{O}$, which could be used to photocatalytic hydrogen evolution reaction. While the photogenerated holes in the $\mathrm{VB}$ of g- $\mathrm{C}_{3} \mathrm{~N}_{4}$ can be transferred to the $\mathrm{VB}$ of $\mathrm{Ag}_{2} \mathrm{O}$, and the holes are consumed by TEOA. Thereby achieving the purpose of charge orientation separation.

Inspired by the research of $\mathrm{NiO}$ modified $\mathrm{TiO}_{2}$, Yu's group reported that $\mathrm{Ni}(\mathrm{OH})_{2}$ as cocatalysts decorated on $\mathrm{TiO}_{2}$ [19], g- $\mathrm{C}_{3} \mathrm{~N}_{4}$ [46] and CdS [171] for enhanced photocatalytic $\mathrm{H}_{2}$ production activity which was synthetized by a simple precipitation reaction. $\mathrm{Ni}(\mathrm{OH})_{2}$ is an outstanding and universal material as cocatalysts for photocatalytic water splitting under visible-light irradiation. The rate of $\mathrm{H}_{2}$ evolution even is higher than Pt as cocatalyst deposited on CdS.

Our group [169] reported that plate-like $\mathrm{Co}(\mathrm{OH})_{2}$ was incorporated onto the surface of $\mathrm{TiO}_{2}$ nanosheets as an effective cocatalyst to increase the rate of $\mathrm{H}_{2}$ production for water splitting. The sample of $0.5 \mathrm{~mol} \% \mathrm{Co}(\mathrm{OH})_{2} / \mathrm{TiO}_{2}$ shows a maximum hydrogen rate with $746.93 \mu \mathrm{mol} \cdot \mathrm{g}^{-1} \cdot \mathrm{h}^{-1}$, which is 20 times higher than pure $\mathrm{TiO}_{2}$ nanosheets, along with a good stability for photocatalytic $\mathrm{H}_{2}$ evolution upon continuous irradiation of 14 h. As shown in Fig. 12(c, d), the reason for photocatalytic performance improvement is the plate-like $\mathrm{Co}(\mathrm{OH})_{2}$ act as electron collector to facilitate the migration of electron from $\mathrm{TiO}_{2}$ to the plate-like $\mathrm{Co}(\mathrm{OH})_{2}$, and reduce partial Co $2 p$ to Co atoms, the as-formed $\mathrm{Co}^{0}$ species can work as a co-catalyst, therefore improve the separation efficiency and lifetime of the photoexcited electrons and holes.

The layered double hydroxide (LDH) can be represented by the general formula $\left[\mathrm{M}^{2+}{ }_{1-x} \mathrm{M}^{3+}{ }_{x}(\mathrm{OH})_{2}\right]\left(\mathrm{A}^{n-}\right)_{x / n} \cdot \mathrm{mH}_{2} \mathrm{O}$, where $\mathrm{M}^{2+}$ and $\mathrm{M}^{3+}$ are divalent and trivalent metal cations respectively and $\mathrm{A}^{n-}$ is the interlayer anion compensating for the positive charge of the brucite-like layers [172-176]. LDHs materials have been studied in photocatalysis due to their low cost, high photocatalytic activity and controllable electronic structure. For photocatalytic water splitting $\mathrm{H}_{2}$ evolution, Zhang et al. [177] synthesized CdSe/ZnCr-LDH nanohybrids via an electrostatically derived self-assemble process, which exhibited a high photocatalytic hydrogen generation rate of $2196 \mu \mathrm{mol} \cdot \mathrm{h}^{-1} \cdot \mathrm{g}^{-1}$ with an AQY of $51.3 \%$ for $\mathrm{H}_{2}$ generation under visible-light irradiation. The $\mathrm{ZnCr}-\mathrm{LDH}$ as cocatalyst was also applied with g- $\mathrm{C}_{3} \mathrm{~N}_{4}$, which is effective in enhancing the photocatalytic hydrogen generation activity, the $\mathrm{H}_{2}$ production rate reached $155.7 \mu \mathrm{mol} \cdot \mathrm{h}^{-1} \cdot \mathrm{g}^{-1}$. ZnCr-LDH acts as an important role in improving the activity of photocatalytic hydrogen production by water splitting. As shown in Fig. 13, the electrons transfer from the $\mathrm{CB}$ of g- $\mathrm{C}_{3} \mathrm{~N}_{4}$ to the lower-lying $\mathrm{CB}$ of $\mathrm{ZnCr}$ - $\mathrm{LDH}$, the holes in the $\mathrm{VB}$ of $\mathrm{ZnCr}-\mathrm{LDH}$ move to the upper-lying $\mathrm{VB}$ of $\mathrm{g}-\mathrm{C}_{3} \mathrm{~N}_{4}$, leading to an efficient separation of electrons and holes [178].

\subsection{Metal sulfides}

To date, many metal sulfides, such as MoS2 [30,184-188], $\mathrm{NiS}[45,51,184,189], \mathrm{Cu}_{x} \mathrm{~S}$ [190-196], $\mathrm{Co}_{3} \mathrm{~S}_{4}$ [197], FeS [198], $\mathrm{Ag}_{2} \mathrm{~S}$ [199], $\mathrm{Bi}_{2} \mathrm{~S}_{3}$ [200], $\mathrm{WS}_{2}$ [201], $\mathrm{Co}_{3} \mathrm{Mo}_{7} \mathrm{~S}$ [15] and NiMoS $[202,203]$, have been reported in photocatalytic $\mathrm{H}_{2}$ evolution 


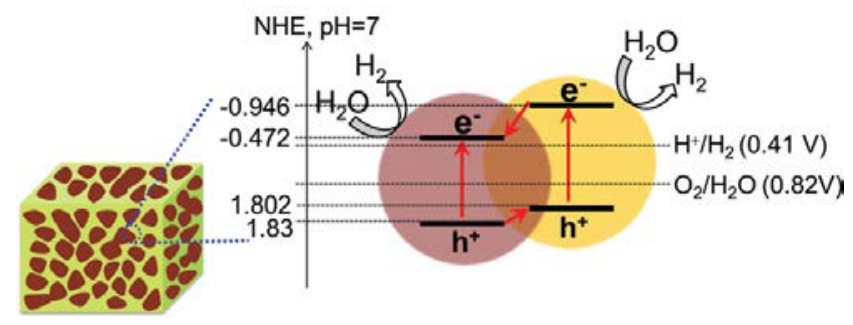

Fig. 13. Schematic model for the band structure of the $\mathrm{ZnCr}-\mathrm{LDH} / \mathrm{g}-\mathrm{C}_{3} \mathrm{~N}_{4}$ nanohybrids. From Ref. [178].

reaction. $\mathrm{MoS}_{2}$ is an efficient cocatalyst on variety of semiconductor photocatalysts. Meng's group [184] load $\mathrm{MoS}_{2}$ on $\mathrm{ZnIn}_{2} \mathrm{~S}_{4}$ via hydrothermal method. Loading $\mathrm{MoS}_{2}$ on $\mathrm{ZnIn}_{2} \mathrm{~S}_{4}$ as a cocatalyst improved the rate of $\mathrm{H}_{2}$ evolution by up to 28 times. In 2016, Li and co-worker [55] reported on the synthesis of novel bilayer junctions by assembling metallic $\mathrm{MoS}_{2}$ monolayers selectively and chemically on oxygen-deficient $\left(\mathrm{Bi}_{12} \mathrm{O}_{17}\right)$ end-faces of $\mathrm{Bi}_{12} \mathrm{O}_{17} \mathrm{Cl}_{2}$ monolayers via oxygen-vacancy (OV) chemistry. Under visible light irradiation, the photocatalytic $\mathrm{H}_{2}$ evolution rate reached $33 \mathrm{mmol} \cdot \mathrm{h}^{-1} \cdot \mathrm{g}^{-1}$ with an AQY of $36 \%$ at $420 \mathrm{~nm}$. Furthermore, the band structures of $\mathrm{Bi}_{12} \mathrm{O}_{17} \mathrm{Cl}_{2}$ and $\mathrm{MoS}_{2}$ were shown in Fig. 14, $\mathrm{Bi}_{12} \mathrm{O}_{17} \mathrm{Cl}_{2}$ in the photoexcited $\mathrm{Bi}_{12} \mathrm{O}_{17} \mathrm{Cl}_{2}-\mathrm{MoS}_{2}$ generated electrons and transferred from $\mathrm{Bi}_{12} \mathrm{O}_{17} \mathrm{Cl}_{2}$ to $\mathrm{MoS}_{2}$ in photocatalytic $\mathrm{H}_{2}$ evolution process. In addition, in $\mathrm{Bi}_{12} \mathrm{O}_{17} \mathrm{Cl}_{2}$, internal electric field can drive electrons to $\left(\mathrm{Bi}_{12} \mathrm{O}_{17}\right)$ end-faces and the holes to $\left(\mathrm{Cl}_{2}\right)$ end-faces, achieving the atomic-level steering of charge separation.

Very recently, Xu et al. [188] found that $\mathrm{MoS}_{2}$ could be facilely modulated through phosphorus doping, which can act as a cocatalyst and embedded onto the CdS nanorod for not only facilitating the separation of the charge carriers, but also promoting the separation efficiency of the charge carriers. The 3 wt.\% CdS/P-MoS2 sample exhibited a higher photocatalytic hydrogen evolution rate of $8.86 \mathrm{mmol} \cdot \mathrm{h}^{-1} \cdot \mathrm{g}^{-1}$ with the AQY of $32.4 \%$ at $420 \mathrm{~nm}$.

In most reports, the NiS cocatalysts are generally decorated on semiconductors to synthetize hybrid photocatalysts. The $\mathrm{NiS} / \mathrm{g}-\mathrm{C}_{3} \mathrm{~N}_{4}$ heterojunction was formed by one-step calcination method [189], hydrothermal method [45] and ion-exchange procedure [51]. All of the photocatalysts exhibited good photocatalytic hydrogen production performance in TEOA solution under visible light illumination, which is 250 times and 56 times higher than pure g- $\mathrm{C}_{3} \mathrm{~N}_{4}$, respectively. This remarkable enhancement in the photocatalytic activity resulted from NiS cocatalyst, which acted as an important role in the $\mathrm{NiS} / \mathrm{g}-\mathrm{C}_{3} \mathrm{~N}_{4}$ photocatalyst by boosting the electron separation and transfer from the $\mathrm{CB}$ g- $\mathrm{C}_{3} \mathrm{~N}_{4}$ to the surface $\mathrm{NiS}$, the reactions are presented in equations (2) and (3):

$$
\begin{gathered}
\mathrm{NiS}+e^{-}+\mathrm{H}^{+} \rightarrow \mathrm{HNiS} \\
\mathrm{HNiS}+e^{-}+\mathrm{H}^{+} \rightarrow \mathrm{NiS}+\mathrm{H}_{2}
\end{gathered}
$$

Furthermore, NiS was also decorated on $\mathrm{TiO}_{2}$ [21], CdS [204,205] and CdZnS [206] to enhance the catalytic activity.

The CuS/ZnS photocatalyst was prepared via a simple hydrothermal and cation exchange reaction between $\mathrm{ZnS}[\mathrm{en}]_{0.5}$ nanosheets and $\mathrm{Cu}\left(\mathrm{NO}_{3}\right)_{2}$ by Zhang in 2011 [191]. The optimal CuS loading content on $\mathrm{ZnS}$ is $2 \mathrm{~mol} \%$ and the corresponding rate of $\mathrm{H}_{2}$ production is $4147 \mu \mathrm{mol} \cdot \mathrm{h}^{-1} \cdot \mathrm{g}^{-1}$ with an AQY of $20 \%$ at $420 \mathrm{~nm}$. As shown in Fig. 15(a), the photoinduced interfacial charge transfer (IFCT) from the $\mathrm{ZnS}$ VB to CuS causes partial reduction of $\mathrm{CuS}$ to $\mathrm{Cu}_{2} \mathrm{~S}$, the holes in the VB of $\mathrm{ZnS}$ could be consumed by the sacrificial agents. The IFCT will retard the recombination of photoinduced electrons and holes due to the space separation and lead to $\mathrm{H}_{2}$ evolution. The $\mathrm{CuS} / \mathrm{Cu}_{2} \mathrm{~S}$ acts as an electron sink and cocatalyst to boost the separation and transfer of electrons from $\mathrm{ZnS}$ VB to $\mathrm{CuS} / \mathrm{Cu}_{2} \mathrm{~S}$, where $\mathrm{H}^{+}$is reduced to $\mathrm{H}_{2}$. The steps of electron transfer in this catalytic $\mathrm{H}_{2}$ evolution process under visible light irradiation are listed is as the following equations:

$$
\begin{gathered}
\mathrm{CuS} / \mathrm{ZnS}+h v \rightarrow \mathrm{CuS}\left(e^{-}\right) / \mathrm{ZnS}\left(h^{+}\right) \\
2 \mathrm{CuS}+2 e^{-} \rightarrow \mathrm{Cu}_{2} \mathrm{~S}+\mathrm{S}^{2-} \\
\mathrm{Cu}_{2} \mathrm{~S}+2 \mathrm{H}^{+}+\mathrm{S}^{2-} \rightarrow 2 \mathrm{CuS}+\mathrm{H}_{2}
\end{gathered}
$$

In 2017, Zhang and co-workers [190] reported that a self-optimizing bifunctional core-shell $\mathrm{CdS} / \mathrm{Cu}_{2} \mathrm{~S}$ heterojunction was synthesized via solvothermal method for photocatalytic hydrogen evolution under visible light irradiation in which the $\mathrm{Cu}_{2} \mathrm{~S}$ acts as cocatalyst. Compared with pure $\mathrm{CdS}$, the rate of photocatalytic $\mathrm{H}_{2}$ production has improved almost 25 times, the maximum rate of hydrogen production reached 14.4 mmol.h ${ }^{-1} \cdot \mathrm{g}^{-1}$ with an AQY of $19.5 \%$ at $420 \mathrm{~nm}$. Fig. $15(\mathrm{~b}, \mathrm{c}$ ) illustrated the mechanism for photocatalytic $\mathrm{H}_{2}$ production by
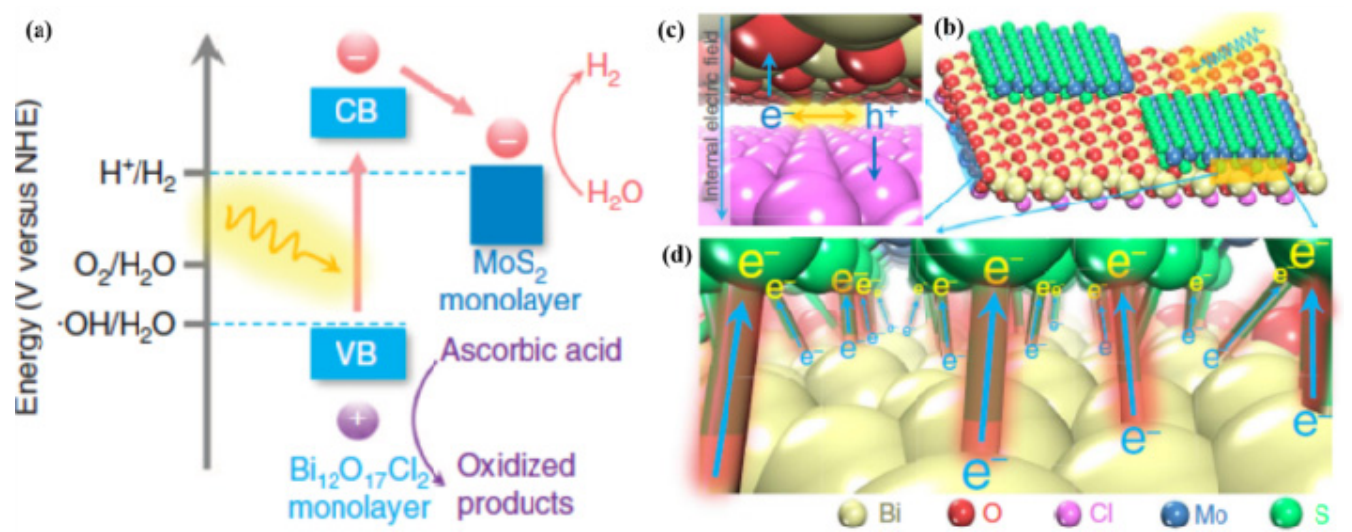

Fig. 14. (a) Band alignments in $\mathrm{Bi}_{12} \mathrm{O}_{17} \mathrm{Cl}_{2}$ and $\mathrm{MoS}_{2}$. Schematic illustration of the crystal structure of $\mathrm{Bi}_{12} \mathrm{O}_{17} \mathrm{Cl}_{2}-\mathrm{MoS}_{2}(\mathrm{~b})$ and of the charge flow processes within $\mathrm{Bi}_{12} \mathrm{O}_{17} \mathrm{Cl}_{2}-\mathrm{MoS}_{2}$, including the electron-hole separation within $\mathrm{Bi}_{12} \mathrm{O}_{17} \mathrm{Cl}_{2}$ (c) and the interfacial electron transfer from $\mathrm{Bi}_{12} \mathrm{O}_{17} \mathrm{Cl}_{2}$ to $\mathrm{MoS}_{2}$ along the Bi-S bonds (d). From Ref. [55]. 

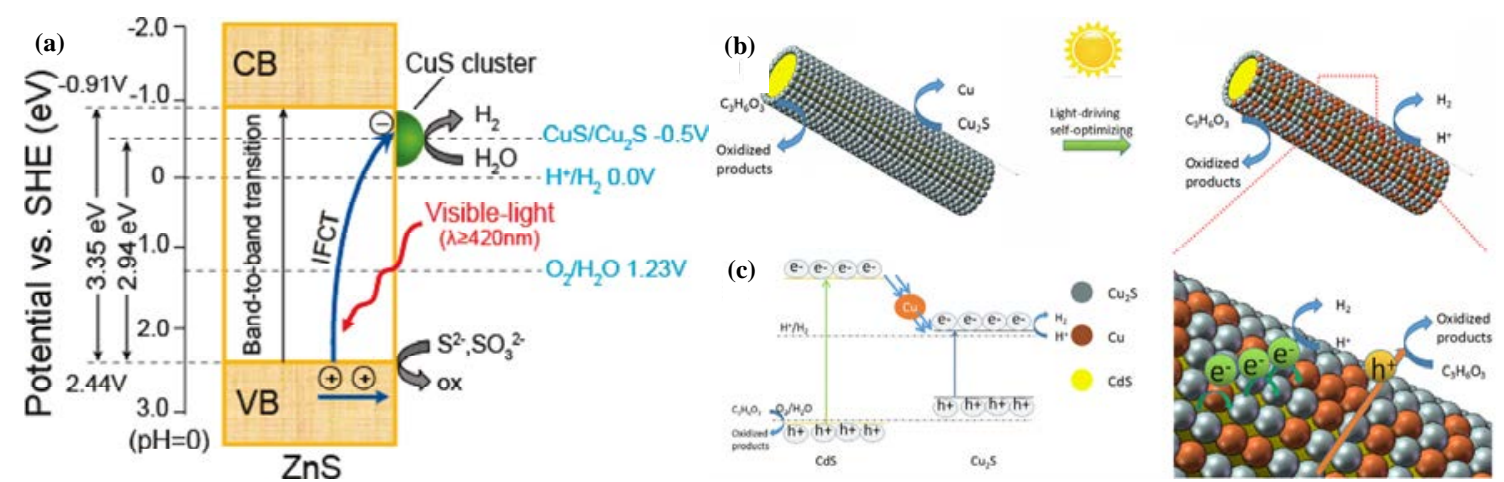

Fig. 15. (a) Schematic illustration for visible light induced interfacial charge transfer from the VB of $\mathrm{ZnS}$ to the CuS clusters in CuS/ZnS system as the proposed mechanism for photocatalytic $\mathrm{H}_{2}$ production. From Ref. [191] (b) Schematic illustration of light-driving self-optimizing process on $\mathrm{CdS} / \mathrm{Cu}_{2} \mathrm{~S}$ core-shell hybrid. (c) Schematic illustration of photocatalytic HER process on Cu@CdS/Cu2S core-shell hybrid. From Ref. [190].
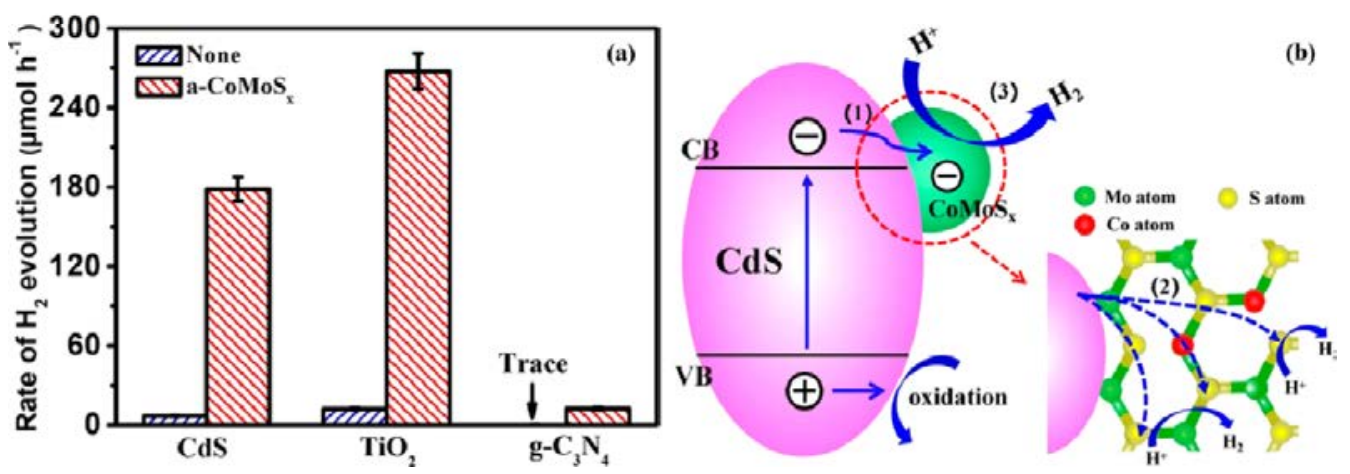

Fig. 16. (a) Photocatalytic performance of typical photocatalytic materials before and after a-CoMoS $x_{x}$ modification. (b) Schematic diagram illustrating the photocatalytic $\mathrm{H}_{2}$-evolution mechanism of a-CoMoS$x / \mathrm{CdS}$ photocatalysts: (1) the rapid capture of photogenerated electrons by a-CoMoS ${ }_{x ;}(2)$ the effective transfer of photogenerated electrons from the capture sites to active sites; (3) the efficient interfacial $\mathrm{H}_{2}$-evolution reactions on the a-CoMoS active sites. From Ref. [207].

$\mathrm{CdS} / \mathrm{Cu}_{2} \mathrm{~S}$. Under visible light irradiation, $e^{--} h^{+}$pairs were generated in CdS core, partial photo-electrons participate in $\mathrm{H}_{2}$ evolution, while the others reduce $\mathrm{Cu}_{2} \mathrm{~S}$ into $\mathrm{Cu}^{0}$. The electrons transfer from the $\mathrm{CB}$ of $\mathrm{CdS}$ to $\mathrm{Cu}_{2} \mathrm{~S} / \mathrm{Cu}^{0}$, the $\mathrm{Cu}_{2} \mathrm{~S}$ acts as active sites for photocatalytic $\mathrm{H}_{2}$ production, while the light-reduced $\mathrm{Cu}^{0}$ accelerates the separation process of photo-generated electrons and holes by forming heterojunction.

In recent years, many studies have reported that the bimetallic sulfide play an important role in enhancing the photocatalytic $\mathrm{H}_{2}$ evolution. Yu's group [207] found that the CoMoS co-catalyst could significantly improve the $\mathrm{H}_{2}$ production activity of CdS photocatalyst, the rate of photocatalytic $\mathrm{H}_{2}$ evolution is $178.5 \mu \mathrm{mol} \cdot \mathrm{h}^{-1}$, which is higher than that of pure $\mathrm{CdS}$, $\mathrm{MoS}_{2} / \mathrm{CdS}$ and $\mathrm{Co} / \mathrm{CdS}$ by 26.3, 3.1 and 7.5 times, respectively. Furthermore, the CoMoS co-catalyst acts as a universal cocatalyst to improve the photocatalytic $\mathrm{H}_{2}$ evolution activity of other semiconductors, such as $\mathrm{TiO}_{2}$ and $\mathrm{g}-\mathrm{C}_{3} \mathrm{~N}_{4}$, their corresponding rate of is shown in Fig. 16. The exceptional visible light photocatalytic $\mathrm{H}_{2}$ evolution performance is due to the presence of CoMoS, which acts as an efficient cocatalyst to prompt the transportation of the electrons for $\mathrm{H}_{2}$ production, the detail process is described as the following 3 steps: (1) under visible light irritation, the CoMoS first work as an electron capturer to prompt the transportation of the electrons from the CB of CdS; (2) then play as an electron transfer mediator to efficiently transport the electrons; (3) finally serves as the activity site for
$\mathrm{H}_{2}$ production to boost the rate of photo-reduction of water.

Similar to $\mathrm{MoS}_{2}, \mathrm{Cu}_{x} \mathrm{~S}$, NiS and $\mathrm{CoMoS}_{x}$, there are also many diverse metal sulfide cocatalysts which can decorated on semiconductors to effectively increase photocatalytic $\mathrm{H}_{2}$-evolution activity, such as $\mathrm{Ag}_{2} \mathrm{~S}$ [199], $\mathrm{Bi}_{2} \mathrm{~S}_{3}$ [200], NiMoS [202,203], $\mathrm{Co}_{3} \mathrm{Mo}_{7} \mathrm{~S}$ [15], $\mathrm{Zn}_{0.23} \mathrm{Cd}_{0.77} \mathrm{~S}$ [208] and $\mathrm{ZnIn}_{2} \mathrm{~S}_{4}$ [209], the application of metal sulfide co-catalysts for photocatalytic $\mathrm{H}_{2}$ evolution is summarized in Table 6.

\subsection{Carbon-based materials}

Common cocatalysts are mostly metal or their compounds loaded on semiconductor photocatalysts. However, metal-free carbon nanomaterials, which have many unique properties to be applications in the photocatalysts field, such as large work function, excellent electron receptors, large surface area and novel morphologies. In addition, they are very cheap, stable, abundant in earth and non-toxic. Many studies have shown that carbon-based materials can be used as an effective cocatalyst or an excellent conductor cocatalyst to enhance the activity of catalytic hydrogen production. Carbon-based materials including carbon quantum dots (CQDs) [27,211-213], graphene [116,214,215] and carbon nanotube (CNT) [50,216-221], and carbon-based material can act as cocatalysts in photocatalytic hydrogen production process, the related researches were summarized and showed in Table 7. 
The CQDs were extensively studied due to some superior performances include the long-term stability, tunable size and band gap and size dependent photoluminescence. In 2018, Wang and co-workers [213] prepared tubular CQD-implanted g- $\mathrm{C}_{3} \mathrm{~N}_{4}$ heterostructures (CCTs) via thermal polymerization of freeze-dried urea and CQDs precursor. The photocatalyst exhibited efficient photocatalytic activity with a high $\mathrm{H}_{2}$ evolution rate of $3538.3 \mu \mathrm{mol} \cdot \mathrm{h}^{-1} \cdot \mathrm{g}^{-1}$ under visible-light irradiation and

Table 6

Summary of metal sulfide as cocatalysts in the photocatalyst systems for $\mathrm{H}_{2}$ evolution.

\begin{tabular}{|c|c|c|c|c|c|c|c|}
\hline Photocatalyst & Cocatalyst & Light source & $\begin{array}{c}\text { Weight } \\
\text { (mg) }\end{array}$ & Solution & Activity & AQY & Year/Ref. \\
\hline 3 wt. $\%$ CdS/P-MoS 2 & $\mathrm{MoS}_{2}$ & $300 \mathrm{~W}$ Xe lamp $(\lambda \geq 420 \mathrm{~nm})$ & 5 & $\begin{array}{c}50 \mathrm{~mL} 0.35 \mathrm{M} \\
\mathrm{Na}_{2} \mathrm{~S} / 0.25 \mathrm{M} \mathrm{Na}_{2} \mathrm{SO}_{3}\end{array}$ & $8.86 \mathrm{mmol} \cdot \mathrm{h}^{-1} \cdot \mathrm{g}^{-1}$ & $\begin{array}{c}32.4 \% \\
(420 \mathrm{~nm})\end{array}$ & 2019 [188] \\
\hline 6 wt. $\% \mathrm{MoS}_{2} / \mathrm{Cu}-\mathrm{ZnIn}_{2} \mathrm{~S}_{4}$ & $\mathrm{MoS}_{2}$ & $300 \mathrm{~W}$ Xe lamp $(\lambda \geq 420 \mathrm{~nm})$ & 50 & $\begin{array}{c}250 \mathrm{~mL} 0.1 \mathrm{M} \text { ascor- } \\
\text { bic acid }\end{array}$ & $5463 \mu \mathrm{mol} \cdot \mathrm{h}^{-1} \cdot \mathrm{g}^{-1}$ & $\begin{array}{c}13.6 \% \\
(420 \mathrm{~nm})\end{array}$ & 2017 [187] \\
\hline 0.5 wt. $\% \mathrm{MoS}_{2} / g-\mathrm{C}_{3} \mathrm{~N}_{4}$ & $\mathrm{MoS}_{2}$ & $300 \mathrm{~W}$ Xe lamp $(\lambda \geq 420 \mathrm{~nm})$ & 50 & $\begin{array}{c}120 \mathrm{~mL} 25 \text { vol\% } \\
\text { methanol }\end{array}$ & $867.6 \mu \mathrm{mol} \cdot \mathrm{h}^{-1} \cdot \mathrm{g}^{-1}$ & - & 2018 [185] \\
\hline 1 wt. $\% \mathrm{MoS}_{2} / \mathrm{ZnIn}_{2} \mathrm{~S}_{4}$ & $\mathrm{MoS}_{2}$ & $300 \mathrm{~W}$ Xe lamp $(\lambda \geq 420 \mathrm{~nm})$ & 80 & $\begin{array}{l}80 \mathrm{~mL} 12.5 \mathrm{vol} \% \\
\text { lactic acid }\end{array}$ & $343 \mu \mathrm{mol} \cdot \mathrm{h}^{-1}$ & $\begin{array}{c}3.85 \% \\
(420 \mathrm{~nm})\end{array}$ & $2017[186]$ \\
\hline 2 wt. $\%$ CdS $/ \mathrm{MoS}_{2}$ & $\mathrm{MoS}_{2}$ & $300 \mathrm{~W}$ Xe lamp $(\lambda \geq 420 \mathrm{~nm})$ & 20 & $\begin{array}{c}80 \mathrm{~mL} 10 \text { vol\% lactic } \\
\text { acid }\end{array}$ & $493.1 \mu \mathrm{mol} \cdot \mathrm{h}^{-1}$ & $\begin{array}{c}28.5 \% \\
(420 \mathrm{~nm})\end{array}$ & $2017[30]$ \\
\hline 60 wt. $\% \mathrm{MoS}_{2} / \mathrm{TiO}_{2}$ & $\mathrm{MoS}_{2}$ & $300 \mathrm{~W}$ Xe lamp $(\lambda \geq 320 \mathrm{~nm})$ & 4 & $\begin{array}{c}80 \mathrm{~mL} 0.35 \mathrm{M} \\
\mathrm{Na}_{2} \mathrm{~S} / 0.25 \mathrm{M} \mathrm{Na}_{2} \mathrm{SO}_{3}\end{array}$ & $1.68 \mathrm{mmol} \cdot \mathrm{h}^{-1} \cdot \mathrm{g}^{-1}$ & - & 2015 [210] \\
\hline 0.375 wt. $\% \mathrm{MoS}_{2} / \mathrm{ZnIn}_{2} \mathrm{~S}_{4}$ & $\mathrm{MoS}_{2}$ & $300 \mathrm{~W}$ Xe lamp $(\lambda \geq 420 \mathrm{~nm})$ & 100 & $\begin{array}{c}150 \mathrm{~mL} 10 \text { vol\% } \\
\text { lactic acid }\end{array}$ & $\begin{array}{c}8.047 \\
\mathrm{mmol} \cdot \mathrm{h}^{-1} \cdot \mathrm{g}^{-1}\end{array}$ & - & $2014[184]$ \\
\hline $\mathrm{MoS}_{2} / \mathrm{Bi}_{12} \mathrm{O}_{17} \mathrm{Cl}_{2}$ & $\mathrm{MoS}_{2}$ & 300 W Xe lamp $(\lambda \geq 420 \mathrm{~nm})$ & 10 & $\begin{array}{c}80 \mathrm{~mL} 0.3 \mathrm{M} \text { ascorbic } \\
\text { acid }\end{array}$ & $33 \mathrm{mmol} \cdot \mathrm{h}^{-1} \cdot \mathrm{g}^{-1}$ & $\begin{array}{c}36 \% \\
(420 \mathrm{~nm})\end{array}$ & $2016[55]$ \\
\hline $\mathrm{NiS} / \mathrm{g}-\mathrm{C}_{3} \mathrm{~N}_{4}$ & $\mathrm{NiS}$ & $300 \mathrm{~W}$ Xe lamp $(\lambda \geq 420 \mathrm{~nm})$ & 50 & $80 \mathrm{~mL} 15$ vol\% TEOA & $29.68 \mu \mathrm{mol} \cdot \mathrm{h}^{-1}$ & - & 2018 [189] \\
\hline $1.5 \mathrm{~mol} \% \mathrm{NiS} / \mathrm{g}-\mathrm{C}_{3} \mathrm{~N}_{4}$ & $\mathrm{NiS}$ & 300 W Xe lamp $(\lambda \geq 420 \mathrm{~nm})$ & 100 & $\begin{array}{c}100 \mathrm{~mL} 10 \mathrm{vol} \% \\
\text { TEOA }\end{array}$ & $44.77 \mu \mathrm{mol} \cdot \mathrm{h}^{-1}$ & - & $2014[51]$ \\
\hline $1.1 \% \mathrm{NiS} / \mathrm{C}_{3} \mathrm{~N}_{4}$ & $\mathrm{NiS}$ & 300 W Xe lamp $(\lambda \geq 420 \mathrm{~nm})$ & 100 & $\begin{array}{c}100 \mathrm{~mL} 15 \text { vol } \% \\
\text { TEOA }\end{array}$ & $48.2 \mu \mathrm{mol} \cdot \mathrm{h}^{-1}$ & $\begin{array}{l}1.1 \%(420 \mathrm{~nm}) \\
1.9 \%(440 \mathrm{~nm})\end{array}$ & $2013[45]$ \\
\hline $7 \mathrm{~mol} \% \mathrm{NiS} / \mathrm{TiO}_{2}$ & $\mathrm{NiS}$ & 300 W Xe lamp & 200 & $\begin{array}{c}100 \mathrm{~mL} 30 \text { vol\% } \\
\text { lactic acid }\end{array}$ & $698 \mu \mathrm{mol} \cdot \mathrm{h}^{-1} \cdot \mathrm{g}^{-1}$ & - & $2012[21]$ \\
\hline $\mathrm{NiS} / \mathrm{CdS}$ & $\mathrm{NiS}$ & $300 \mathrm{~W}$ Xe lamp $(\lambda \geq 420 \mathrm{~nm})$ & 300 & $\begin{array}{c}100 \mathrm{~mL} 30 \text { vol\% } \\
\text { lactic acid }\end{array}$ & $2.18 \mathrm{mmol} \cdot \mathrm{h}^{-1}$ & $51.3 \%(420 \mathrm{~nm})$ & 2010 [204] \\
\hline $\mathrm{NiS} / \mathrm{CdS}$ & $\mathrm{NiS}$ & $300 \mathrm{~W}$ Xe lamp $(\lambda \geq 400 \mathrm{~nm})$ & 100 & $\begin{array}{c}110 \mathrm{~mL} 1.22 \mathrm{M} \text { lactic } \\
\text { acid }\end{array}$ & $5.98 \mathrm{mmol} \cdot \mathrm{h}^{-1} \cdot \mathrm{g}^{-1}$ & $69.9 \%(420 \mathrm{~nm})$ & 2018 [205] \\
\hline $0.01 \% \mathrm{~g}-\mathrm{C}_{3} \mathrm{~N}_{4} / \mathrm{WS}_{2}$ & $\mathrm{WS}_{2}$ & 300 W Xe lamp $(\lambda \geq 420 \mathrm{~nm})$ & 50 & $\begin{array}{l}80 \mathrm{~mL} 25 \text { vol\% } \\
\text { methanol }\end{array}$ & $101 \mu \mathrm{mol} \cdot \mathrm{h}^{-1} \cdot \mathrm{g}^{-1}$ & - & 2015 [201] \\
\hline $30 \% \mathrm{CdS} / \mathrm{Cu}_{2} \mathrm{~S}$ & $\mathrm{Cu}_{2} \mathrm{~S}$ & $300 \mathrm{~W}$ Xe lamp $(\lambda \geq 420 \mathrm{~nm})$ & 20 & $\begin{array}{c}80 \mathrm{~mL} 10 \text { vol\% lactic } \\
\text { acid/0.5 M K } \mathrm{HPO}_{4}\end{array}$ & $14.4 \mathrm{mmol} \cdot \mathrm{h}^{-1} \cdot \mathrm{g}^{-1}$ & $19.5 \%(420 \mathrm{~nm})$ & ) 2017 [190] \\
\hline $2 \mathrm{~mol} \% \mathrm{CuS} / \mathrm{ZnS}$ & $\mathrm{CuS}$ & $350 \mathrm{~W}$ Xe lamp $(\lambda \geq 420 \mathrm{~nm})$ & 50 & $\begin{array}{c}50 \mathrm{~mL} 0.35 \mathrm{M} \\
\mathrm{Na}_{2} \mathrm{~S} / 0.25 \mathrm{M} \mathrm{Na}_{2} \mathrm{SO}_{3}\end{array}$ & $4147 \mu \mathrm{mol} \cdot \mathrm{h}^{-1} \cdot \mathrm{g}^{-1}$ & $20 \%(420 \mathrm{~nm})$ & 2011 [191] \\
\hline 2 wt. $\% \mathrm{CuS} / \mathrm{g}-\mathrm{C}_{3} \mathrm{~N}_{4}$ & $\mathrm{CuS}$ & 300 W Xe lamp $(\lambda \geq 420 \mathrm{~nm})$ & 50 & $\begin{array}{c}200 \mathrm{~mL} 20 \text { vol } \% \\
\text { TEOA }\end{array}$ & $17.2 \mu \mathrm{mol} \cdot \mathrm{h}^{-1} \cdot \mathrm{g}^{-1}$ & - & 2017 [192] \\
\hline 3 wt. $\% \mathrm{CuS} / \mathrm{Zn}_{0.8} \mathrm{Cd}_{0.2} \mathrm{~S}$ & $\mathrm{CuS}$ & 500 W Xe lamp & 200 & $\begin{array}{c}200 \mathrm{~mL} 0.35 \mathrm{M} \\
\mathrm{Na}_{2} \mathrm{~S} / 0.25 \mathrm{M} \mathrm{Na}_{2} \mathrm{SO}_{3}\end{array}$ & $2792 \mu \mathrm{mol} \cdot \mathrm{h}^{-1} \cdot \mathrm{g}^{-1}$ & $37.6 \%(420 \mathrm{~nm})$ & 2013 [194] \\
\hline $3 \mathrm{~mol} \% \mathrm{CuS} / \mathrm{CdS}$ & $\mathrm{CuS}$ & $500 \mathrm{~W}$ Xe lamp & 200 & $\begin{array}{c}200 \mathrm{~mL} 0.35 \mathrm{M} \\
\mathrm{Na}_{2} \mathrm{~S} / 0.25 \mathrm{M} \mathrm{Na}_{2} \mathrm{SO}_{3}\end{array}$ & $332 \mu \mathrm{mol} \cdot \mathrm{h}^{-1} \cdot \mathrm{g}^{-1}$ & - & 2013 [195] \\
\hline 1 wt. $\% \mathrm{CuS} / \mathrm{TiO}_{2}$ & $\mathrm{CuS}$ & 500 W Xe lamp & 50 & $\begin{array}{l}80 \mathrm{~mL} 50 \text { vol } \% \\
\text { methanol }\end{array}$ & $570 \mu \mathrm{mol} \cdot \mathrm{h}^{-1}$ & - & 2013 [196] \\
\hline $\mathrm{Cu}_{2} \mathrm{~S} / \mathrm{CdS}-0.05$ & $\mathrm{Cu}_{2} \mathrm{~S}$ & 300 W Xe lamp $(\lambda \geq 420 \mathrm{~nm})$ & 200 & $\begin{array}{c}190 \mathrm{~mL} 0.35 \mathrm{M} \\
\mathrm{Na}_{2} \mathrm{~S} / 0.25 \mathrm{M} \mathrm{Na}_{2} \mathrm{SO}_{3}\end{array}$ & $400.1 \mu \mathrm{mol} \cdot \mathrm{h}^{-1}$ & $9.5 \%(420 \mathrm{~nm})$ & 2015 [193] \\
\hline $5 \% \mathrm{Ag}_{2} \mathrm{~S} / \mathrm{g}-\mathrm{C}_{3} \mathrm{~N}_{4}$ & $\mathrm{Ag}_{2} \mathrm{~S}$ & $12 \mathrm{~W}$ UV-LEDs $(\lambda \geq 420 \mathrm{~nm})$ & 50 & $\begin{array}{c}50 \text { mL } 25 \text { vol } \% \\
\text { methanol }\end{array}$ & $10 \mu \mathrm{mol} \cdot \mathrm{h}^{-1}$ & - & 2014 [199] \\
\hline 6 wt. $\% \mathrm{Bi}_{2} \mathrm{~S}_{3} / \mathrm{TiO}_{2}$ & $\mathrm{Bi}_{2} \mathrm{~S}_{3}$ & UV Hg pen-lamp $\lambda=254 \mathrm{~nm}$ & 50 & $\begin{array}{c}200 \mathrm{~mL} 50 \mathrm{vol} \% \\
\text { methanol }\end{array}$ & $2460 \mu \mathrm{mol} \cdot \mathrm{h}^{-1} \cdot \mathrm{g}^{-1}$ & - & 2016 [200] \\
\hline $\mathrm{g}-\mathrm{C}_{3} \mathrm{~N}_{4} @ \mathrm{ZnIn}_{2} \mathrm{~S}_{4}$ & $\mathrm{ZnIn}_{2} \mathrm{~S}_{4}$ & $300 \mathrm{~W}$ Xe lamp $(\lambda \geq 420 \mathrm{~nm})$ & 50 & $50 \mathrm{~mL} 20 \mathrm{vol} \%$ TEOA & $2.78 \mathrm{mmol} \cdot \mathrm{h}^{-1} \cdot \mathrm{g}^{-1}$ & $3.80 \%(420 \mathrm{~nm})$ & 2018 [209] \\
\hline $10 \% \mathrm{Co}_{3} \mathrm{Mo}_{7} \mathrm{~S} / \mathrm{Cd}_{0.5} \mathrm{Zn}_{0.5} \mathrm{~S}$ & $\mathrm{Co}_{3} \mathrm{Mo}_{7} \mathrm{~S}$ & $300 \mathrm{~W}$ Xe lamp $(\lambda \geq 420 \mathrm{~nm})$ & 50 & $\begin{array}{c}100 \mathrm{~mL} 0.35 \mathrm{M} \\
\mathrm{Na}_{2} \mathrm{~S} / 0.25 \mathrm{M} \mathrm{Na}_{2} \mathrm{SO}_{3}\end{array}$ & $188.65 \mu \mathrm{mol} \cdot \mathrm{h}^{-1}$ & $\begin{array}{c}16.72 \% \\
(420 \mathrm{~nm})\end{array}$ & 2019 [15] \\
\hline $\mathrm{Cu}_{1.94} \mathrm{~S} / \mathrm{Zn}_{0.23} \mathrm{Cd}_{0.77} \mathrm{~S}$ & $\mathrm{Zn}_{0.23} \mathrm{Cd}_{0.77} \mathrm{~S}$ & 300 W Xe lamp $(\lambda \geq 420 \mathrm{~nm})$ & 20 & $\begin{array}{c}50 \mathrm{~mL} 0.1 \mathrm{M} \\
\mathrm{Na}_{2} \mathrm{~S} / 0.1 \mathrm{M} \mathrm{Na}_{2} \mathrm{SO}_{3}\end{array}$ & $7735 \mu \mathrm{mol} \cdot \mathrm{h}^{-1} \cdot \mathrm{g}^{-1}$ & $26.4 \%(420 \mathrm{~nm})$ & 2016 [208] \\
\hline 3 wt. $\%$ NiMoS/CdS & NiMoS & 300 W Xe lamp $(\lambda \geq 420 \mathrm{~nm})$ & 5 & $\begin{array}{c}20 \mathrm{~mL} 15 \text { vol\% lactic } \\
\text { acid }\end{array}$ & $\begin{array}{c}185.4 \\
\mathrm{mmol} \cdot \mathrm{h}^{-1} \cdot \mathrm{g}^{-1}\end{array}$ & $\begin{array}{c}21.82 \% \\
(420 \mathrm{~nm})\end{array}$ & 2017 [203] \\
\hline $1 \% \mathrm{NiMoS} / \mathrm{CdS}$ & NiMoS & $300 \mathrm{~W}$ Xe lamp & 50 & $\begin{array}{c}60 \mathrm{~mL} 0.35 \mathrm{M} \\
\mathrm{Na}_{2} \mathrm{~S} / 0.25 \mathrm{M} \mathrm{Na}_{2} \mathrm{SO}_{3}\end{array}$ & $24 \mathrm{mmol} \cdot \mathrm{h}^{-1} \cdot \mathrm{g}^{-1}$ & $17.3 \%(435 \mathrm{~nm})$ & 2016 [202] \\
\hline CoMoS/CdS & CoMoS & $300 \mathrm{~W}$ Xe lamp $(\lambda \geq 420 \mathrm{~nm})$ & 50 & $\begin{array}{c}80 \mathrm{~mL} 10 \text { vol\% lactic } \\
\text { acid }\end{array}$ & $178.5 \mu \mathrm{mol} \cdot \mathrm{h}^{-1}$ & - & 2018 [207] \\
\hline
\end{tabular}


Table 7

Summary of carbon-based materials as cocatalysts photocatalyst system for $\mathrm{H}_{2}$ evolution.

\begin{tabular}{|c|c|c|c|c|c|c|c|}
\hline Photocatalyst & Cocatalyst & Light source & $\begin{array}{l}\text { Weight } \\
\text { (mg) }\end{array}$ & Solution & Activity & $\mathrm{AQY}$ & Year/Ref. \\
\hline 10 wt. \% CNNS/CQDs & CQDs & $\begin{array}{l}1000 \text { W Xe lamp } \\
(\lambda \geq 420 \mathrm{~nm})\end{array}$ & 10 & $\begin{array}{l}10 \mathrm{~mL} 20 \text { vol\% } \\
\text { methanol }\end{array}$ & $219.5 \mu \mathrm{mol} \cdot \mathrm{h}^{-1} \cdot \mathrm{g}^{-1}$ & $\begin{array}{l}0.024 \%(700 \mathrm{~nm}) \\
0.034 \%(808 \mathrm{~nm})\end{array}$ & 2015 [211] \\
\hline 10 wt. $\%$ CDs $/ g-C_{3} N_{4}$ & CDs & $3 \mathrm{~W}$ LEDs $(\lambda \geq 355 \mathrm{~nm})$ & 50 & $\begin{array}{c}80 \mathrm{~mL} 10 \mathrm{vol} \% \text { lactic } \\
\text { acid }\end{array}$ & $183.0 \mu \mathrm{mol} \cdot \mathrm{h}^{-1}$ & - & 2017 [212] \\
\hline CQDs $/ \mathrm{TiO}_{2}$ & CQDs & 300 W Xe lamp & 20 & $\begin{array}{l}60 \mathrm{~mL} 20 \text { vol\% } \\
\text { methanol }\end{array}$ & $7.9 \mu \mathrm{mol} \cdot \mathrm{h}^{-1}$ & - & 2019 [27] \\
\hline CCTs/CQDs & CQDs & $\begin{array}{c}300 \text { W Xe lamp } \\
(\lambda \geq 400 \mathrm{~nm})\end{array}$ & 50 & $\begin{array}{l}60 \mathrm{~mL} 20 \text { vol\% } \\
\text { methanol }\end{array}$ & $3538.3 \mu \mathrm{mol} \cdot \mathrm{h}^{-1} \cdot \mathrm{g}^{-1}$ & $10.94 \%(420 \mathrm{~nm})$ & 2018 [213] \\
\hline 1.0 wt. $\%$ graphene $/ g-C_{3} N_{4}$ & graphene & $\begin{array}{l}350 \text { W Xe lamp } \\
(\lambda \geq 400 \mathrm{~nm})\end{array}$ & 80 & 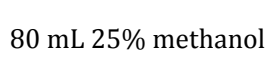 & $451 \mu \mathrm{mol} \cdot \mathrm{h}^{-1} \cdot \mathrm{g}^{-1}$ & $2.6 \%$ & $2011[214]$ \\
\hline $\mathrm{Cu}_{2} \mathrm{O} / \mathrm{RGO}-2$ w.t $\%$ & RGO & $\begin{array}{c}300 \text { W Hg lamp } \\
(\lambda \geq 400 \mathrm{~nm})\end{array}$ & 20 & $\begin{array}{l}20 \mathrm{~mL} 20 \text { vol\% } \\
\text { methanol }\end{array}$ & $64.3 \mu \mathrm{mol} \cdot 6 \mathrm{~h}^{-1} \cdot \mathrm{g}^{-1}$ & - & 2019 [215] \\
\hline 0.1 wt. $\%$ MWCNT-TiO 2 & MWCNT & $\begin{array}{l}\text { Mercury Vapour (Hg) } \\
\text { lamp (200-800 nm) }\end{array}$ & 5 & $50 \mathrm{~mL} 5$ vol\% glycerol & $8.8 \mathrm{mmol} \cdot \mathrm{h}^{-1} \cdot \mathrm{g}^{-1}$ & - & 2018 [216] \\
\hline $\begin{array}{l}\mathrm{Zn}_{0.83} \mathrm{Cd}_{0.17} \mathrm{~S} / \mathrm{CNTs}-0.25 \\
\text { wt. } \%\end{array}$ & CNTs & 500 W Xe lamp & 1 & $\begin{array}{c}40 \mathrm{~mL} 0.1 \mathrm{M} \\
\mathrm{Na}_{2} \mathrm{~S} / 0.02 \mathrm{M} \mathrm{Na}_{2} \mathrm{SO}_{3}\end{array}$ & $5.41 \mathrm{mmol} \cdot \mathrm{h}^{-1} \cdot \mathrm{g}^{-1}$ & - & 2014 [217] \\
\hline $\mathrm{CNT} @ \mathrm{Ni}_{3} \mathrm{~S}_{2}$ & CNT & $\begin{array}{c}300 \text { W Xe lamp } \\
(\lambda \geq 420 \mathrm{~nm})\end{array}$ & 50 & $\begin{array}{c}100 \mathrm{~mL} 15 \mathrm{vol} \% \\
\text { TEOA and } 200 \mathrm{mg} \text { ErY }\end{array}$ & $5.32 \mathrm{mmol} \cdot \mathrm{h}^{-1} \cdot \mathrm{g}^{-1}$ & $11.1 \%(420 \mathrm{~nm})$ & 2012 [218] \\
\hline 0.25 wt.\% CNT/Cd $\mathrm{d}_{0.1} \mathrm{Zn}_{0.9} \mathrm{~S}$ & CNT & $\begin{array}{c}300 \text { W Xe lamp } \\
(\lambda \geq 420 \mathrm{~nm})\end{array}$ & 50 & $\begin{array}{c}80 \mathrm{~mL} 0.35 \mathrm{M} \\
\mathrm{Na}_{2} \mathrm{~S} / 0.25 \mathrm{M} \mathrm{Na}_{2} \mathrm{SO}_{3}\end{array}$ & $78.2 \mu \mathrm{mol} \cdot \mathrm{h}^{-1}$ & $7.9 \%(420 \mathrm{~nm})$ & 2012 [219] \\
\hline 0.2 wt. $\%$ CNT/g- $\mathrm{C}_{3} \mathrm{~N}_{4}$ & CNT & $\begin{array}{l}300 \text { W Xe lamp } \\
(\lambda \geq 420 \mathrm{~nm})\end{array}$ & 100 & $\begin{array}{l}100 \mathrm{~mL} 10 \text { vol\% } \\
\text { TEOA }\end{array}$ & $39.4 \mu \mathrm{mol} \cdot \mathrm{h}^{-1}$ & - & $2014[50]$ \\
\hline $\mathrm{CNT} / \mathrm{g}-\mathrm{C}_{3} \mathrm{~N}_{4}$ & CNT & $\begin{array}{c}10 \text { LED lamps }(\lambda \geq 420 \\
\mathrm{nm})\end{array}$ & 100 & $50 \mathrm{~mL} 10$ vol\% TEOA & $654.8 \mu \mathrm{mol} \cdot \mathrm{h}^{-1} \cdot \mathrm{g}^{-1}$ & $37.9 \%(420 \mathrm{~nm})$ & 2017 [220] \\
\hline $\mathrm{Zn}_{0.83} \mathrm{Cd}_{0.17} \mathrm{~S} / \mathrm{CNTs}$ & CNT & 500 W Xe lamp & 10 & $\begin{array}{c}40 \mathrm{~mL} 0.1 \mathrm{M} \\
\mathrm{Na}_{2} \mathrm{~S} / 0.02 \mathrm{M} \mathrm{Na}_{2} \mathrm{SO}_{3} \\
\end{array}$ & $6.03 \mathrm{mmol} \cdot \mathrm{h}^{-1} \cdot \mathrm{g}^{-1}$ & - & 2013 [221] \\
\hline
\end{tabular}

AQY was $10.94 \%$ at $420 \mathrm{~nm}$. The integration of CQDs into CCTs optimizes the morphology and band structure, increases visible light absorption and reduces the energy barrier, thus improving the photocatalytic efficiency for $\mathrm{H}_{2}$ evolution. Tang's group [211] reported the CQDs decorated g- $\mathrm{C}_{3} \mathrm{~N}_{4}$ nanosheets via hydrothermal method. The photocatalytic $\mathrm{H}_{2}$ evolution ability was tremendously improved by loading $10 \mathrm{wt}$ \% CQDs as a cocatalyst. This study showed that CQDs/g- $\mathrm{C}_{3} \mathrm{~N}_{4}$ displayed a maximum photocatalytic $\mathrm{H}_{2}$ evolution rate of 219.5 $\mu \mathrm{mol} \cdot \mathrm{h}^{-1} \cdot \mathrm{g}^{-1}$ (UV-Vis) and $50.5 \mu \mathrm{mol} \cdot \mathrm{h}^{-1} \cdot \mathrm{g}^{-1}$ (Vis). In addition, the 10 wt.\% CQDs/g- $\mathrm{C}_{3} \mathrm{~N}_{4}$ has an AQY of $0.024 \%$ at $700 \mathrm{~nm}$ and $0.034 \%$ under $808 \mathrm{~nm}$ laser, the wavelength dependence of EQE clearly shows the extended active range toward the NIR region derived from the higher content CQD loading. The broadband absorption and high photocatalytic activity originate from the interaction of spectral and electronic spectrum, coupling these interactions helps to promote the wide range absorption and photoinduced charge separation Very recently, Sui et al. [27] found that the CQDs modified $\mathrm{TiO}_{2}$ with a majority of (001) facet via a facile method. The CQDs/ $\mathrm{TiO}_{2}-001$ exhibited a superior photocatalytic $\mathrm{H}_{2}$ evolution ability. CQDs loading on the surface of $\mathrm{TiO}_{2}-001$ plays an important role of electron reservoir to trap photogenerated electron, which not only promotes efficient separation of electron-hole pairs, but also prevents the recombination of the photogenerated electron-hole pairs.

Graphene (GO), a two-dimensional macromolecular sheet of carbon atoms with a honeycomb structure, which has a wide applications in photocatalysis due to the outstanding performance with high thermal conductivity, excellent mobility of charge carriers and extremely high specific surface area [222-224]. Yu's group [214] reported the fabrication of graphene/g- $\mathrm{C}_{3} \mathrm{~N}_{4}$ by combined impregnation-chemical reduction strategy. The optimal graphene content was $1.0 \mathrm{wt} . \%$ and the corresponding photocatalytic $\mathrm{H}_{2}$ evolution rate was 451 $\mu \mathrm{mol} \cdot \mathrm{h}^{-1} \cdot \mathrm{g}^{-1}$. The graphene acted as an acceptor of the photogenerated electrons, which could enhance the photocatalytic $\mathrm{H}_{2}$ evolution under visible light irradiation. Zhang et al. [116] reported an in-site process for preparation of $\mathrm{Cu}$ NPs on rGO nanosheet. The $\mathrm{Cu} / \mathrm{rGO}$ composite exhibited superior photocatalytic $\mathrm{H}_{2}$ evolution activity which possesses a hydrogen evolution rate of $59 \mathrm{mmol} \cdot \mathrm{h}^{-1} \cdot \mathrm{g}^{-1}$ in lactic acid solution under visible light irradiation. Significantly, the $\mathrm{Cu} / \mathrm{rGO}$ composite still have hydrogen production rate of 2.76 and 1.96 $\mathrm{mmol} \cdot \mathrm{h}^{-1} \cdot \mathrm{g}^{-1}$ with the wavelength of $800 \mathrm{~nm}$ and $900 \mathrm{~nm}$, respectively. The rGO co-catalyst acts as an electron mediator for enhancing the photoinduced carrier separation efficiency and promote the restrain of $e^{--} h^{+}$recombination. To date, Zhang et al. [215] have prepared $\mathrm{Cu}_{2} \mathrm{O}-\mathrm{RGO}$ heterojunction with oxygen vacancy through hydrothermal method. Compared with pure $\mathrm{Cu}_{2} \mathrm{O}$, the rate of $2 \mathrm{wt} \% \mathrm{Cu}_{2} \mathrm{O}-\mathrm{RGO}$ heterojunction photocatalytic $\mathrm{H}_{2}$ evolution was $64.3 \mu \mathrm{mol} \cdot \mathrm{h}^{-1} \cdot \mathrm{g}^{-1}$, which was 5 times higher than that of pure $\mathrm{Cu}_{2} \mathrm{O}\left(12.8 \mu \mathrm{mol} \cdot \mathrm{h}^{-1} \cdot \mathrm{g}^{-1}\right)$. The $\mathrm{RGO}$ as co-catalyst loading on $\mathrm{Cu}_{2} \mathrm{O}$ to form heterojunction could enhance a broader absorbance under visible light and promote a faster electron transfer, which lead to the improved photocatalytic $\mathrm{H}_{2}$ production activity.

Recently, Reddy and co-workers [216] prepared CNT/TiO 2 has enhanced photocatalytic $\mathrm{H}_{2}$ evolution. After loading CNT on $\mathrm{TiO}_{2}$, the photocatalytic $\mathrm{H}_{2}$ evolution rate could be increased 


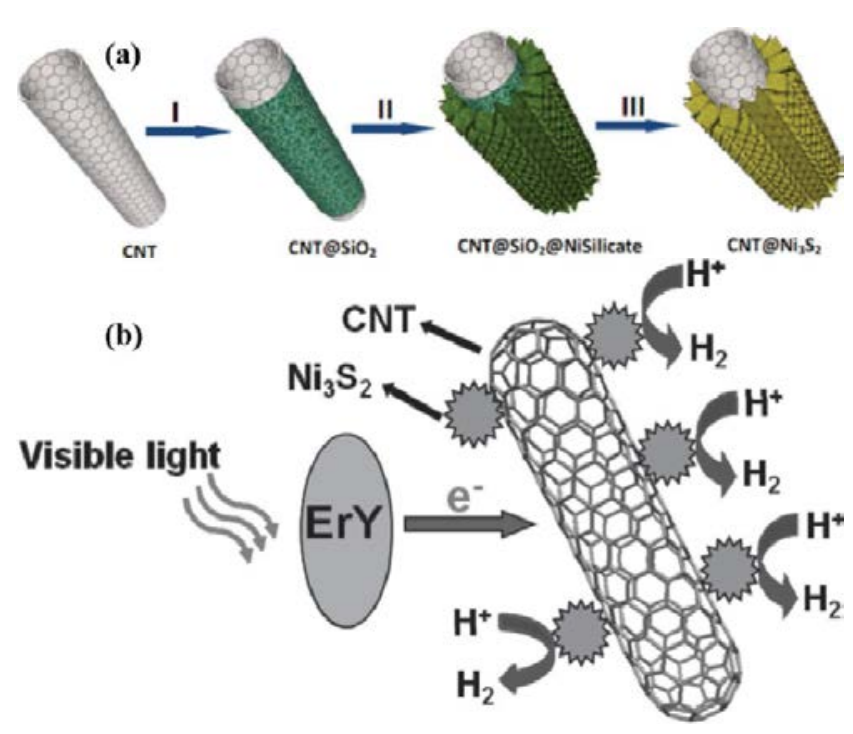

Fig. 17. (a) Schematic illustration of the formation of $\mathrm{Ni}_{3} \mathrm{~S}_{2}$ ultrathin nanosheets supported on CNT backbone (CNT@Ni $\mathrm{N}_{2}$ ) by a multi-step conversion route: (I) uniform coating of a silica layer on CNT; (II) in-situ formation of nickel silicate shell on the silica layer; and (III) chemical conversion of nickel silicate into $\mathrm{Ni}_{3} \mathrm{~S}_{2}$ to form $\mathrm{CNT} @ \mathrm{Ni}_{3} \mathrm{~S}_{2}$ with simultaneous elimination of the intermediate silica layer. (b) Schematic illustration of photocatalytic $\mathrm{H}_{2}$ production over the $\mathrm{CNT} @ \mathrm{Ni}_{3} \mathrm{~S}_{2}$ structure. From Ref. [218].

from 1.3 to $8.8 \mathrm{mmol} \cdot \mathrm{h}^{-1} \cdot \mathrm{g}^{-1}$. Yu et al. [219] investigation revealed that the CNT could serve as a highly efficient co-catalyst

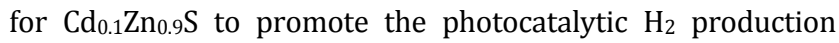
under visible-light irradiation. The optimum rate of $\mathrm{H}_{2}$ evolution is $78.2 \mu \mathrm{mol} \cdot \mathrm{h}^{-1}$ with an AQY of $7.9 \%$ at $420 \mathrm{~nm}$, exceeding that of pure $\mathrm{Cd}_{0.1 \mathrm{Zn}} \mathrm{Zn}_{0.9} \mathrm{~S}$ by more than 3.3 times. Chen's group [50] found that introduce $0.2 \mathrm{wt} . \% \mathrm{CNT}$ into g- $\mathrm{C}_{3} \mathrm{~N}_{4}$ could enhance its photocatalytic activity for $\mathrm{H}_{2}$ evolution under visible-light irradiation, the highest $\mathrm{H}_{2}$ evolution rate is 39.4 $\mu \mathrm{mol} \cdot \mathrm{h}^{-1}$, which is 2.4 times higher than the value obtained with pure $\mathrm{g}-\mathrm{C}_{3} \mathrm{~N}_{4}$. In 2012, Lou's group [218] reported that CNT@Ni3 $\mathrm{S}_{2}$ nanostructures for photocatalytic $\mathrm{H}_{2}$ evolution which composed of $\mathrm{Ni}_{3} \mathrm{~S}_{2}$ nanosheets grown on carbon nanotube (CNT) backbone via multi-step transformation route, the synthesis procedure of $\mathrm{CNT} @ \mathrm{Ni}_{3} \mathrm{~S}_{2}$ nanostructures is shown in Fig. 17. Under visible light irritation, the rate of photocatalytic $\mathrm{H}_{2}$ evolution is $5.32 \mathrm{mmol} \cdot \mathrm{h}^{-1} \cdot \mathrm{g}^{-1}$ with an AQY of $11.1 \%$ at 420 $\mathrm{nm}$, which is superior to pure $\mathrm{Ni}_{3} \mathrm{~S}_{2}$. The high photocatalytic hydrogen production activity is due to the sensitization effect of ErY for effective visible light absorption, which would then be transformed into excited stated. The photoexcited electrons in ErY are quickly transferred to the $\mathrm{Ni}_{3} \mathrm{~S}_{2}$ nanosheets due to the presence of the CNT backbone. Then the protons partake in the reduction reaction to product $\mathrm{H}_{2}$ on $\mathrm{Ni}_{3} \mathrm{~S}_{2}$ (Fig. 17). In this photocatalytic system, CNT not only acts as co-catalyst to collect and transfer electrons, but also provides the active sites for $\mathrm{H}_{2}$ evolution.

\section{Dual cocatalysts for photocatalytic $\mathrm{H}_{2}$ evolution}

\subsection{Randomly distributed}

Efficient charge separation is an effective way to improve the photocatalytic hydrogen evolution performance. The hydrogen production efficiency of water splitting over single photocatalyst is limited by the rapid recombination of photoexcited charge carriers. Cocatalyst loading can effectively accelerate the surface reaction to promote the water splitting process [225]. The electron-hole pairs are separated under the light irradiation, and the photoexcited electrons are the main active species that are used to reduce the proton to $\mathrm{H}_{2}$. Various reductive cocatalysts have been broadly studied to transport electrons to water protons. Noble metals such as Pt and Pd with higher work function and lower impedance are usually primary candidates to act as reductive cocatalysts to transfer the electrons to protons. The higher work function equally to the lower Fermi level results in the separated electrons inclines to the noble metals, while the lower impedance can insure a faster electron mobility, which can prompt the photoinduced electrons transfer to the water proton. However, noble metals are suffering from high cost and low abundance in the earth. The emergence of various non-noble species has played an important role in the development of reductive cocatalyst. The nucleus on photocatalytic $\mathrm{H}_{2}$ production are concentrated on the half reduction reaction of water splitting. The holes are unnecessary factor to charge separation. Oxidative cocatalyst, such as some metal oxides, $\mathrm{CoO}_{x}, \mathrm{IrO}_{2}$ etc., has a tendency to capture holes, while reductive cocatalysts acting as electrons trapping agents to pull photoinduced electrons away from the photogenerated charges [29,226-228]. The applications of dual-cocatalysts photocatalyst systems for $\mathrm{H}_{2}$ evolution are summarized in Table 8.

In 2016, Yu's group [229] reported that synergistic effect of dual amorphous cocatalysts, in which the Ti(IV)-hole cocatalyst and $\mathrm{Ni}(\mathrm{II})$-electron cocatalyst were loaded on $\mathrm{CdS}$ to improve the photocatalytic $\mathrm{H}_{2}$ evolution activity under visible light irradiation. The maximum photocatalytic $\mathrm{H}_{2}$ evolution rate is $3437.95 \mu \mathrm{mol} \cdot \mathrm{h}^{-1} \cdot \mathrm{g}^{-1}$, which is 2.1 times higher than that of pure CdS. The reason for the high performance is that when both of Ti(IV) and Ni(II) cocatalysts are simultaneously modified on the CdS surface, the Ni(II) cocatalyst can first act as an effective trapping site to accept the electrons, and then act as the reduction active site to boost the $\mathrm{H}_{2}$ production reactions on the surface, while the Ti(IV) cocatalyst act as hole cocatalyst to quickly transfer the holes and boot the oxidation reaction (Fig. 18).

Wang et al. [230] reported the co-loading of graphene quantum dots (GQDs) and PdS dual cocatalysts on ZnCdS surface by hydrothermal and in-situ chemical deposition method for efficient photocatalytic $\mathrm{H}_{2}$ evolution. The GQDs/ZnCdS/PdS hybrids exhibited the highest $\mathrm{H}_{2}$ evolution rate of $517 \mu \mathrm{mol} \cdot \mathrm{h}^{-1}$ with an AQY of $22.4 \%$ at $420 \mathrm{~nm}$, which were 15,7 and 1.7 times higher than that of pure ZnCdS, GQDs/ZnCdS and ZnCdS/PdS, respectively.

Li's group [32] synthesized ternary structured photocatalyst which is composed by co-loading dual earth-abundant cocatalysts of metallic carbon black(CB) and $\mathrm{NiS}_{2}$ on $\mathrm{CdS}$ via solvothermal and impregnation method. The superior photocatalytic $\mathrm{H}_{2}$ evolution activity of $\mathrm{CdS} / \mathrm{CB} / \mathrm{NiS}_{2}$ is $166.7 \mu \mathrm{mol} \cdot \mathrm{h}^{-1}$. As 
Table 8

Summary of dual-cocatalyst photocatalyst systems for $\mathrm{H}_{2}$ evolution.

\begin{tabular}{|c|c|c|c|c|c|c|c|}
\hline Photocatalyst & Cocatalyst & Light source & $\begin{array}{l}\text { Weight } \\
\text { (mg) }\end{array}$ & Solution & Activity & AQY & Year/Ref. \\
\hline $\mathrm{Pt} / \mathrm{TiO}_{2} / \mathrm{CdS} / \mathrm{Co}_{3} \mathrm{O}_{4}$ & $\mathrm{Pt} / \mathrm{Co}_{3} \mathrm{O}_{4}$ & $300 \mathrm{~W}$ Xe lamp $(\lambda \geq 420 \mathrm{~nm})$ & 30 & $0.2 \mathrm{M} \mathrm{Na}_{2} \mathrm{~S}$ and $\mathrm{Na}_{2} \mathrm{SO}_{3}$ & $2000 \mu \mathrm{I}$ & - & 2017 [231] \\
\hline Pt@CdS/1\%PdS & $\mathrm{Pt} / \mathrm{PdS}$ & $300 \mathrm{~W}$ Xe lamp $(\lambda \geq 400 \mathrm{~nm})$ & 10 & $\begin{array}{c}50 \mathrm{~mL} 0.1 \mathrm{M} \mathrm{Na}_{2} \mathrm{~S} / \\
0.1 \mathrm{M} \mathrm{Na}_{2} \mathrm{SO}_{3}\end{array}$ & $144.8 \mathrm{mmol} \cdot \mathrm{h}^{-1} \cdot \mathrm{g}^{-1}$ & $\begin{array}{c}11.65 \% \\
(420 \mathrm{~nm})\end{array}$ & 2018 [234] \\
\hline $\begin{array}{l}0.019 \text { wt. } \% \\
\mathrm{CoO}_{x} / \mathrm{TiO}_{2} / 0.046 \text { wt. } \% \mathrm{Pt}\end{array}$ & $\mathrm{CoO}_{\mathrm{x}} / \mathrm{Pt}$ & 300 W Xe lamp & 35 & $\begin{array}{l}200 \mathrm{~mL} 15 \text { vol\% } \\
\text { methanol }\end{array}$ & $275.9 \mu \mathrm{mol} \cdot \mathrm{h}^{-1}$ & - & 2016 [235] \\
\hline $\mathrm{Ni}(\mathrm{II})-\mathrm{Ti}(\mathrm{IV}) / \mathrm{CdS}$ & $\mathrm{Ni}(\mathrm{II})-\mathrm{Ti}(\mathrm{IV})$ & 350 W Xe lamp $(\lambda \geq 420 \mathrm{~nm})$ & 50 & $\begin{array}{c}80 \mathrm{~mL} 0.35 \mathrm{M} \mathrm{Na}_{2} \mathrm{~S} / 0.25 \\
\mathrm{M} \mathrm{Na}_{2} \mathrm{SO}_{3}\end{array}$ & $\begin{array}{c}3437.95 \\
\mu \mathrm{mol} \cdot \mathrm{h}^{-1} \cdot \mathrm{g}^{-1}\end{array}$ & - & 2016 [229] \\
\hline GQDs/ZnCdS/PdS & GQDs/PdS & $300 \mathrm{~W}$ Xe lamp $(\lambda \geq 420 \mathrm{~nm})$ & 50 & $\begin{array}{l}100 \mathrm{~mL} 0.5 \mathrm{M} \\
\mathrm{Na}_{2} \mathrm{~S}_{\mathrm{Na}} \mathrm{SO}_{3}\end{array}$ & $517 \mu \mathrm{mol} \cdot \mathrm{h}^{-1}$ & $\begin{array}{c}22.4 \% \\
(420 \mathrm{~nm})\end{array}$ & 2018 [230] \\
\hline $\mathrm{CdS} / \mathrm{CB} / \mathrm{NiS}_{2}$ & $\mathrm{CB} / \mathrm{NiS}_{2}$ & $300 \mathrm{~W}$ Xe lamp $(\lambda \geq 420 \mathrm{~nm})$ & 50 & $\begin{array}{l}80 \mathrm{~mL} 0.25 \mathrm{M} \\
\mathrm{Na}_{2} \mathrm{~S} / \mathrm{Na}_{2} \mathrm{SO}_{3}\end{array}$ & $166.7 \mu \mathrm{mol} \cdot \mathrm{h}^{-1}$ & - & $2017[32]$ \\
\hline $\begin{array}{l}15 \text { wt.\% } \\
\mathrm{MoS}_{2}-\mathrm{Mn}_{0.2} \mathrm{Cd}_{0.8} \mathrm{~S} / \mathrm{MnS}\end{array}$ & $\mathrm{MoS}_{2} / \mathrm{MnS}$ & $300 \mathrm{~W}$ Xe lamp $(\lambda \geq 420 \mathrm{~nm})$ & 50 & $\begin{array}{c}50 \mathrm{~mL} 0.35 \mathrm{M} \mathrm{Na}_{2} \mathrm{~S} / \\
0.25 \mathrm{M} \mathrm{Na}_{2} \mathrm{SO}_{3}\end{array}$ & $995 \mu \mathrm{mol} \cdot \mathrm{h}^{-1}$ & $\begin{array}{c}30.2 \% \\
(420 \mathrm{~nm})\end{array}$ & [236] \\
\hline ZnO-ZnS/graphene & graphene & 300 W Xe lamp & 50 & $100 \mathrm{~mL} 40$ vol\% glycerol & 1070 & - & 237] \\
\hline $\mathrm{CdS} / \mathrm{Au} / \mathrm{g}-\mathrm{C}_{3} \mathrm{~N}_{4}$ & $\mathrm{CdS}$ & Xe lamp $(\lambda \geq$ & 100 & & $19.02 \mu \mathrm{mol} \cdot \mathrm{h}^{-1} \cdot \mathrm{g}^{-1}$ & - & 238] \\
\hline $\mathrm{CsTaWO}_{6} / \mathrm{Au} / \mathrm{g}-\mathrm{C}_{3} \mathrm{~N}_{4}$ & $\mathrm{Au} / \mathrm{g}-\mathrm{C}_{3} \mathrm{~N}_{4}$ & 300 W Xe lamp & 50 & $\begin{array}{l}100 \mathrm{~mL} 20 \text { vol\% } \\
\text { methanol }\end{array}$ & $9.16 \mu \mathrm{mol} \cdot \mathrm{h}^{-1} \cdot \mathrm{g}^{-1}$ & - & 2015 [239] \\
\hline${ }_{3} \mathrm{~N}_{4} / \mathrm{Pt}-\mathrm{TiO}_{2}(70: 30)$ & $\mathrm{Pt} /$ & $300 \mathrm{~W}$ Xe & 100 & $200 \mathrm{~mL} 10 \mathrm{vol} \%$ TEOA & 17 & - & $2012[44]$ \\
\hline $\mathrm{g}-\mathrm{C}_{3} \mathrm{~N}_{4} / \mathrm{Pt} / \mathrm{GO}-0.5 \%$ & $\mathrm{Pt} / \mathrm{GO}$ & $300 \mathrm{~W}$ Xe lamp $(\lambda \geq 420 \mathrm{~nm})$ & 50 & $\begin{array}{c}100 \mathrm{~mL} 20 \text { vol\% } \\
\text { TEOA/50 mg Eosin Y }\end{array}$ & $3.82 \mathrm{mmol} \cdot \mathrm{h}^{-1} \cdot \mathrm{g}^{-1}$ & $\begin{array}{c}9.7 \% \\
(420 \mathrm{~nm})\end{array}$ & $2018[240]$ \\
\hline 2 wt. $\% \mathrm{Ni} / \mathrm{NiO} / \mathrm{g}-\mathrm{C}_{3} \mathrm{~N}_{4}$ & $\mathrm{Ni} / \mathrm{NiO}$ & $300 \mathrm{~W}$ Xe lamp $(\lambda \geq 420 \mathrm{~nm})$ & 50 & $100 \mathrm{~mL} 10 \mathrm{vol} \% \mathrm{TEOA}$ & $10 \mu \mathrm{mol} \cdot \mathrm{h}^{-1}$ & - & 2015 [241] \\
\hline $\mathrm{Ni} / \mathrm{Ni}(\mathrm{OH})_{2} / \mathrm{CdS}$ & $\mathrm{Ni} / \mathrm{Ni}(\mathrm{OH})_{2}$ & $300 \mathrm{~W}$ Xe lamp $(\lambda \geq 400 \mathrm{~nm})$ & 100 & $\begin{array}{c}100 \mathrm{~mL} 30 \text { vol\% metha- } \\
\text { nol }\end{array}$ & $373.5 \mu \mathrm{mol} \cdot \mathrm{h}^{-1}$ & - & 2015 [242] \\
\hline $\begin{array}{l}\text { wt. } \% \mathrm{CuS} / 5 \text { wt. } \% \\
\mathrm{iS} / \mathrm{TiO}_{2}\end{array}$ & $\mathrm{CuS} / \mathrm{NiS}$ & 500 W Xe lamp & 50 & $80 \mathrm{~mL} 50$ vol\% methanol & $80 \mu \mathrm{mol} \cdot \mathrm{h}^{-1}$ & - & [243] \\
\hline $\mathrm{Ni} / \mathrm{CdS} / \mathrm{g}-\mathrm{C}_{3} \mathrm{~N}_{4}$ & $\mathrm{i} / \mathrm{CdS}$ & W Xe & 100 & $100 \mathrm{~mL} 10$ vol\% & 1258.7 & - & [244] \\
\hline $\mathrm{TiO}_{2} / \mathrm{Au} / \mathrm{CdS}$ & $\mathrm{Au} / \mathrm{CdS}$ & 300 W Xe lamp $(\lambda \geq 420 \mathrm{~nm})$ & 100 & $0.1 \mathrm{M} \mathrm{Na}_{2} \mathrm{~S} / 0.1 \mathrm{M} \mathrm{Na}_{2} \mathrm{SO}_{3}$ & $1.81 \mathrm{mmol} \cdot \mathrm{h}^{-1} \cdot \mathrm{g}^{-1}$ & - & 2016 [245] \\
\hline $\mathrm{TiO}_{2} / \mathrm{Au} / \mathrm{CdS}$ & $\mathrm{u} / \mathrm{CdS}$ & $300 \mathrm{~W}$ Xe lamp $(\lambda \geq 400 \mathrm{~nm})$ & 25 & $\begin{array}{c}100 \mathrm{~mL} 0.1 \mathrm{M} \mathrm{Na}_{2} \mathrm{~S} / \\
0.1 \mathrm{M} \mathrm{Na}_{2} \mathrm{SO}_{3}\end{array}$ & $0.76 \mu \mathrm{mol} \cdot \mathrm{h}^{-1} \cdot \mathrm{g}^{-1}$ & - & 2016 [246] \\
\hline $\mathrm{ZnS} / \mathrm{CuS} / \mathrm{CdS}$ & & & 100 & $150 \mathrm{~mL} \mathrm{60mM} \mathrm{Na} 2 \mathrm{~S}$ & $837.6 \mu \mathrm{mol} \cdot \mathrm{h}^{-1} \cdot \mathrm{g}^{-1}$ & - & 2014 [247] \\
\hline $\mathrm{TiO}_{2}-\mathrm{Ni}(\mathrm{OH})_{2} / \mathrm{CNT} / \mathrm{CdS}$ & $\mathrm{CNT} / \mathrm{CdS}$ & $300 \mathrm{~W}$ Xe lamp $(\lambda \geq 400 \mathrm{~nm})$ & 50 & $230 \mathrm{~mL} 8.7 \%$ lactic acid & $12 \mathrm{mmol} \cdot \mathrm{h}^{-1} \cdot \mathrm{g}^{-1}$ & - & 2017 [248] \\
\hline $\mathrm{Cu} @ \mathrm{CuO}-\mathrm{g}-\mathrm{C}_{3} \mathrm{~N}_{4} / \mathrm{MCM}-41$ & $\begin{array}{l}\mathrm{Cu} @ \mathrm{CuO} \\
/ \mathrm{MCM}-41\end{array}$ & $125 \mathrm{~W}$ mercury $(\lambda \geq 400 \mathrm{~nm})$ & 20 & $20 \mathrm{~mL} 17$ vol\% methanol & $750 \mu \mathrm{mol} \cdot 2 \mathrm{~h}^{-1}$ & $24.8 \%$ & 2016 [249] \\
\hline $\mathrm{Cu}-\mathrm{Cu}_{2} \mathrm{O}-\mathrm{CuO} / \mathrm{TiO}_{2}$ & $\begin{array}{l}\mathrm{Cu}-\mathrm{Cu}_{2} \mathrm{O}- \\
\quad \mathrm{CuO}\end{array}$ & 300 W Xe lamp & 40 & $40 \mathrm{~mL} 10$ vol\% methanol & $12779 \mu \mathrm{mol} \cdot \mathrm{h}^{-1} \cdot \mathrm{g}^{-1}$ & - & 2013 [250] \\
\hline $0.5 \% \mathrm{Cr}_{2} \mathrm{O}_{3} / \mathrm{TNS} / \mathrm{MoS}_{2}$ & $\mathrm{MoS}_{2}$ & $300 \mathrm{~W}$ Xe lamp $(\lambda \geq 400 \mathrm{~nm})$ & 50 & $80 \mathrm{~mL} 15$ vol\% TEOA & $217 \mu \mathrm{mol} \cdot \mathrm{h}^{-1}$ & - & 2018 [251] \\
\hline
\end{tabular}

illustrated in Fig. 19(a), under visible light irradiation, the electrons are excited from the VB to the $\mathrm{CB}$ of $\mathrm{CdS}$, then the electrons move to the carbon black due to its intimate interface contact and matched work functions, in which the carbon black acts as an electron mediator to transfer the collected electrons to the $\mathrm{H}_{2}$ evolution active sites on $\mathrm{NiS}_{2}$ owing to its excellent electrical conductivity and carrier mobility properties, leading to the effective separation and transfer of the electron-hole pairs. The effective separation of electron-hole pairs is the key factor in improving the photocatalytic hydrogen production activity.

\subsection{Orientated distributed}

The charge separation can be further promoted by loading reductive and oxidative cocatalysts [232]. The rational design of spatially-separated dual cocatalysts on photocatalysts has aroused an increasing attention. In most cases, the cocatalysts were randomly distributed on the surface of semiconductors, resulting in a random flow of the photogenerated electrons and holes and severe charges recombination. Li's group [233] reported an Pt-PdS/CdS photocatalyst in 2009. Visi- ble-light-driven hydrogen production with the rational simultaneously design of oxidative and reductive cocatalysts could further enhance the photocatalytic activity. Wang et al. [231] have demonstrated the efficiency of $\mathrm{Pt} / \mathrm{TiO}_{2} / \mathrm{CdS} / \mathrm{Co}_{3} \mathrm{O}_{4}$ in hierarchical structure, Pt and $\mathrm{Co}_{3} \mathrm{O}_{4}$ species were spatially decorated on the inner and outer surface of the $\mathrm{TiO}_{2} / \mathrm{CdS}$ heterojunction shell. Its hydrogen production rate is 2000 $\mu \mathrm{mol} \cdot \mathrm{h}^{-1} \cdot \mathrm{g}^{-1}$ under visible light irradiation, which is over 540 times higher than that of $\mathrm{TiO}_{2} / \mathrm{CdS}$ double-shelled hollow spheres under the same conditions. The outstanding $\mathrm{H}_{2}$ evolution rate can be attributed to the efficient separation of photogenerated carriers, transportation and interface reaction. The ultrathin shell thickness could contribute to the fast electron transportation and the synergistic effect of the separately loaded dual cocatalysts (Fig. 19(b)).

Sun et al. [234] synthesized a novel hollow porous CdS photocatalyst with Pd and PdS nanoparticles spatially distributed on the inner and outer surfaces by using microporous zeolites as a hard template. The Pd@CdS/PdS sample exhibits superior visible-light-driven photocatalytic $\mathrm{H}_{2}$ evolution rate up to 144.8 $\mathrm{mmol} \cdot \mathrm{h}^{-1} \cdot \mathrm{g}^{-1}$. This is the highest values among all of the reported CdS-based catalysts. This synthetic approach may be 


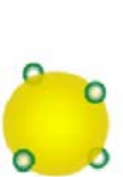

(c)

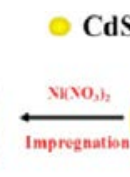

(a)

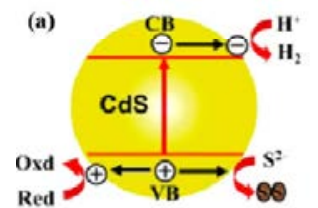

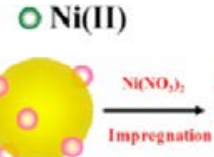

(b)

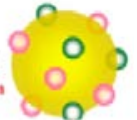

(d)
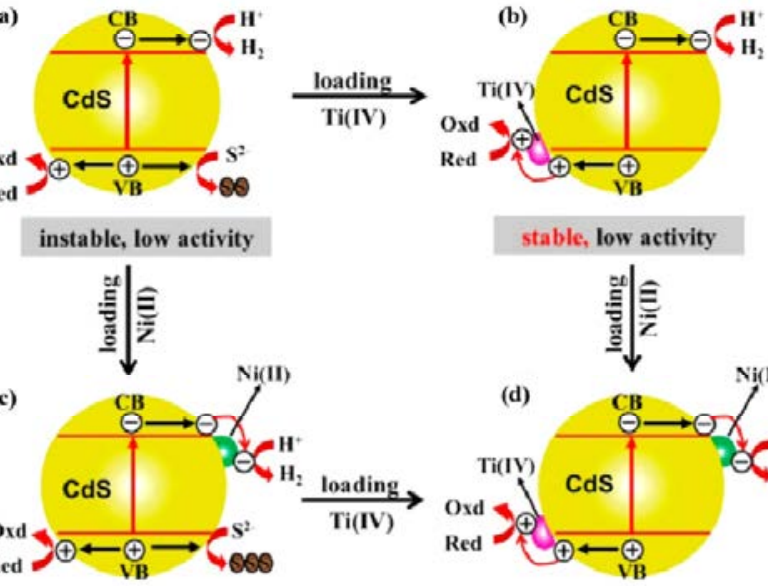

instable, high activity

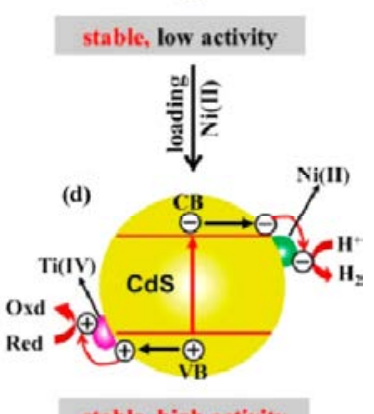

stable, high activity

Fig. 18. Graphical illustration for the synthesis and photocatalytic mechanism of various samples: (a) CdS, (b) Ti(IV)/CdS, (c) Ni(II)/CdS, and (d) Ti(IV)-Ni(II)/CdS. From Ref. [229].

used to fabricate other highly efficient catalysts with spatially

separated cocatalysts. Furthermore, the remarkably enhanced $\mathrm{H}_{2}$ evolution rate can be result from the spatially separated reaction sites in the Pd@CdS/PdS sample, in which the metallic Pd pulls the photoexcited electrons away from CdS while PdS pushes the holes for more thorough oxidation of the sacrificial agent.

In 2016, Zhang and co-worker [235] reported that $\mathrm{CoO}_{x} / \mathrm{TiO}_{2} / \mathrm{Pt}$ photocatalysts produced by template-assisted atomic layer deposition for photocatalytic hydrogen evolution with $\mathrm{CoO}_{x}$ and Pt dual cocatalysts. Fig. 20 showed the reaction process of $\mathrm{CoO}_{x} / \mathrm{TiO}_{2} / \mathrm{Pt}$ photocatalysts with porous hollow $\mathrm{TiO}_{2}$ nanotubes as a semiconductor, which the $\mathrm{Pt}$ and $\mathrm{CoO}_{x}$ deposited on the inner and outer surfaces of the nanotubes, respectively. The highest rate of 0.019 wt. $\% \mathrm{CoO}_{x} / \mathrm{TiO}_{2} / 0.046$ wt.\% Pt photocatalysts is $275.9 \mu \mathrm{mol} \cdot \mathrm{h}^{-1}$, which is almost 5 times higher than that of porous hollow $\mathrm{TiO}_{2}$ nanotubes. The spatially separated dual Pt and $\mathrm{CoO}_{x}$ cocatalysts not only facilitate electron-hole separation but also provide the activity sites for photocatalytic $\mathrm{H}_{2}$ evolution on the surface.

\section{Z-scheme cocatalysts for photocatalytic $\mathrm{H}_{2}$ evolution}

\subsection{Redox mediator Z-scheme photocatalyst system}

An artificial Z-scheme photocatalyst system was inspired by the natural photocatalytic process, which was first reported by
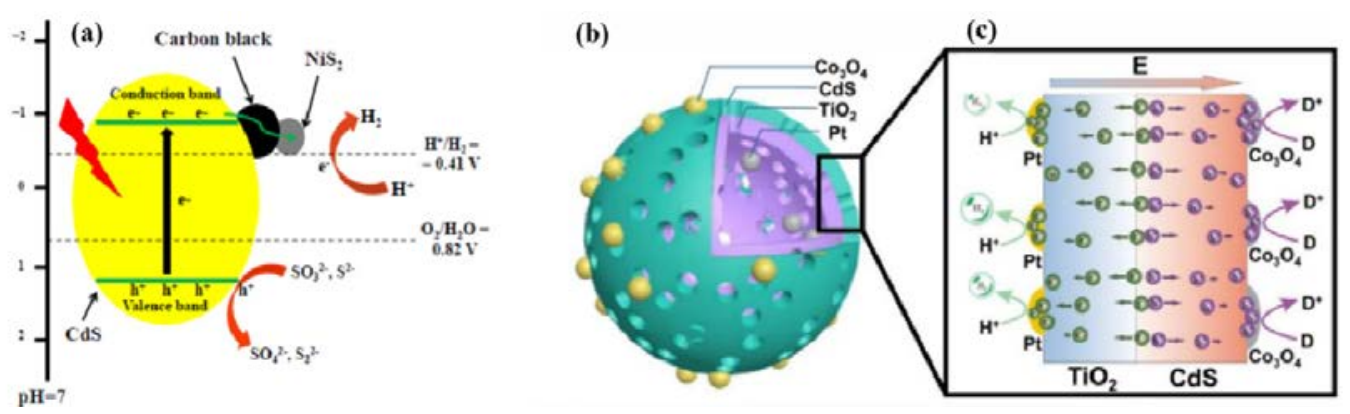

Fig. 19. (a) Proposed photocatalytic $\mathrm{H}_{2}$ evolution and charge transfer mechanisms of CdS/CB/NiS ${ }_{2}$ composite photocatalysts under visible-light irradiation [32]; Illustration of the (b) structure and (c) reaction procedure of double-shelled hollow sphere photocatalyst. From Ref. [231].
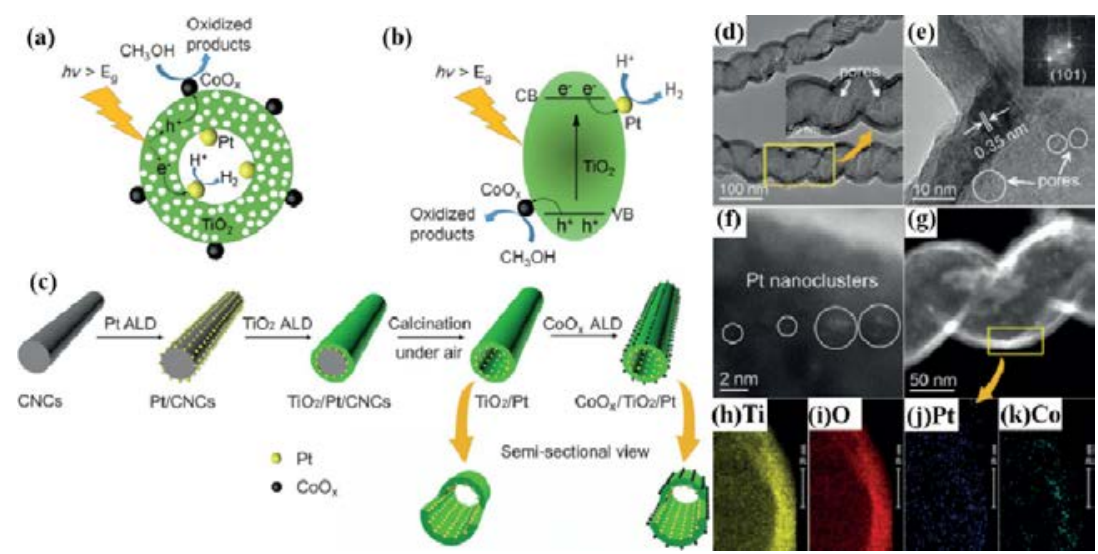

Fig. 20. The proposed mechanism for photocatalytic hydrogen production mediated by $\mathrm{CoO}_{x} / \mathrm{TiO}_{2} / \mathrm{Pt}$. (a) The reaction process. (b) Band structure of the photocatalysts. (c) Synthetic process for $\mathrm{TiO}_{2} / \mathrm{Pt}$ and $\mathrm{CoO}_{x} / \mathrm{TiO}_{2} / \mathrm{Pt}$ photocatalysts by template-assisted ALD. (d) TEM and (e) HRTEM images of

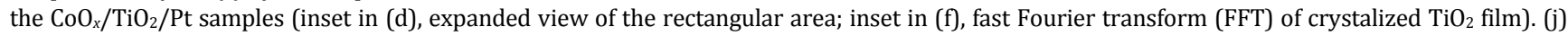
Atomic-resolution HAADF-STEM image of the $\mathrm{CoO}_{x} / \mathrm{TiO}_{2} / \mathrm{Pt}$ samples. (h-k) STEM image and EDS mapping profiles of the rectangular area in panel (h) for (h) titanium, (i) oxygen, (j) platinum, and (k) cobalt. One ALD cycle for Pt and $\mathrm{CoO}_{x}$. From Ref. [235]. 


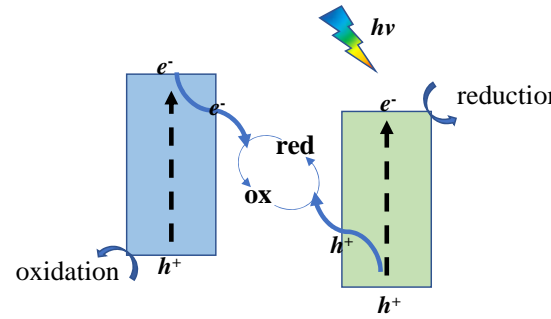

(a) redox mediator Z-scheme

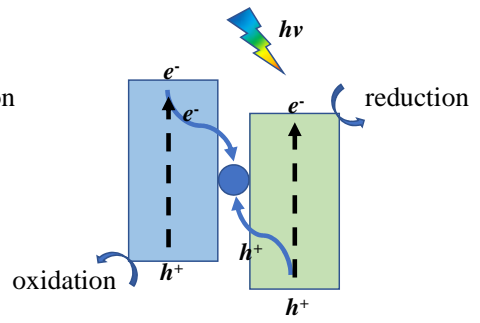

(b) all-solid-state Z-scheme

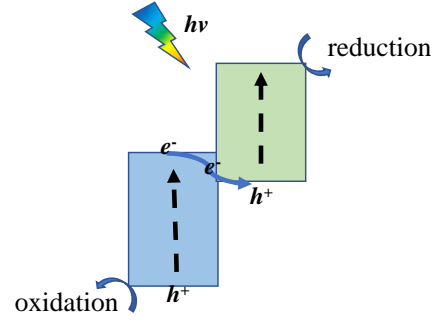

(c) direct Z-scheme

Fig. 21. Schematic illustration of the (a) redox mediator Z-scheme system, (b) all-solid-state Z-scheme system, and (c) direct Z-scheme system.

Bard in 1979 [252]. The schematic illustration of the redox-mediator Z-scheme system is shown in Fig. 21(a), there is no direct contact between the two semiconductors in the Z-scheme system, and the charge carrier transfer is achieved by ion redox shuttles in solution. Both the two semiconductors are photoexcited and generate $e^{-} / h^{+}$pairs in their CB and VB under light irradiation, respectively. And then the photogenerated $e^{-}$ in the CB of PS II is transferred to the VB of PS I via D/A pairs. Finally, $h^{+}$in the VB of PS II participate in oxidation reaction and $e^{-}$in the $\mathrm{CB}$ of PS I participate in reduction reaction on the surface of photocatalysts, respectively. Commonly used D/A pairs are $\mathrm{IO}_{3}-\mathrm{I}^{-}[253], \mathrm{Fe}^{3+} / \mathrm{Fe}^{2+}[254,255], \mathrm{I}_{3}^{-} / \mathrm{I}^{-}$[256], $\mathrm{NO}_{3}-/ \mathrm{NO}_{2}{ }^{-}[257]$, [Co(phen) $\left.)_{3}\right]^{3+/ 2+}$ and $\left[\mathrm{Co}(\mathrm{bpy})_{3}\right]^{2+}[258]$.

In 2016, Ma and co-workers [256] reported a Z-scheme of $\mathrm{Sm}_{2} \mathrm{Ti}_{2} \mathrm{~S}_{2} \mathrm{O}_{5}$ and co-loading with $\mathrm{Pt}$ to generate $\mathrm{H}_{2}$, in which triiodide/iodide $\left(\mathrm{I}_{3}^{-} / \mathrm{I}^{-}\right)$redox couple were used as a shuttle electron mediator. Kudo's group [258] found a Z-scheme system was constructed using a $\mathrm{Ru} / \mathrm{BaTa}_{2} \mathrm{O}_{6}: \mathrm{Ir}, \mathrm{La} \mathrm{H}_{2}$ evolving photocatalyst combined with a $\left[\mathrm{Co}(\mathrm{bpy})_{3}\right]^{2+}$ as electron mediator. The redox potential of $\left[\mathrm{Co}(\mathrm{bpy})_{3}\right]^{2+}$ was reported as $0.32 \mathrm{~V}$, and the value was not affected by $\mathrm{pH}$. Due to the doping of Ir, the wide band gap material has a wider visible light absorption range. Yang et al. [259] revealed a carbon nanodots(CNDs) $/ \mathrm{WO}_{3} \mathrm{Z}$-scheme to improve the photocatalytic performance with $\mathrm{IO}_{3}^{-} / \mathrm{I}^{-}$as shuttle redox mediator. As shown in Fig. 22, photogenerated $e^{-} / h^{+}$pairs tend to recombine, and only a little of them participates in the photocatalytic reactions, which results in low photocatalytic activity. For the Z-scheme system, the photogenerated electrons transferred from the $\mathrm{CB}$ of $\mathrm{WO}_{3}$ to the $\mathrm{VB}$ of $\mathrm{CNDs}$ through $\mathrm{IO}_{3}-/ \mathrm{I}^{-}$as shuttle redox mediator. Therefor the $e^{-}$reduce $\mathrm{H}^{+}$to $\mathrm{H}_{2}$ in the CB of CNDs. Because of the existence of the $\mathrm{IO}_{3}^{-} / \mathrm{I}^{-}$, the $e^{-} / h^{+}$pairs could be

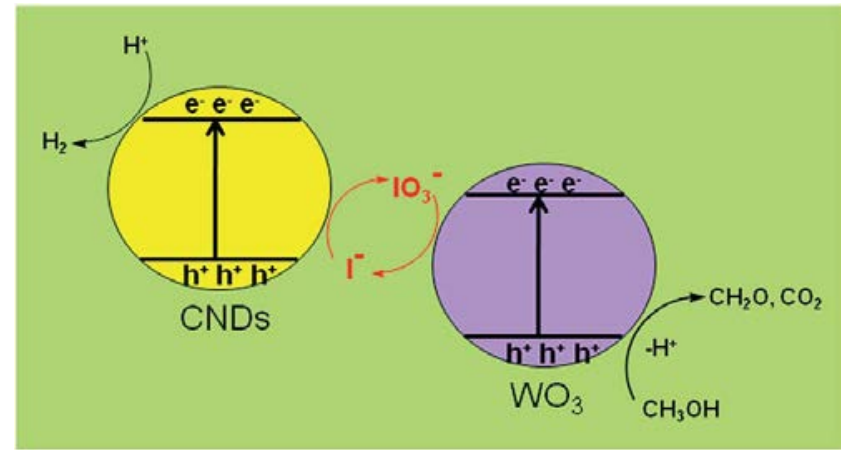

Fig. 22. Schematic diagram of the Z-scheme photocatalytic mechanism of $\mathrm{CNDs} / \mathrm{WO}_{3}$ photocatalyst. From Ref. [259]. effectively separated without direct contact between the two semiconductors, thereby improving the photocatalytic activity of water splitting.

\subsection{All-solid-state photocatalyst system}

However, the redox-mediator Z-scheme photocatalytic system has some drawbacks. Namely, the redox mediator-induced back reactions are thermodynamically favorable, and can easily occur because the photogenerated electrons and holes with strong redox power are consumed by the shuttle redox ion pairs. In addition, the light-shielding effect, feasibility confined in solution systems and solution $\mathrm{pH}$ sensitivity could be limited its application in the field of photocatalytic water splitting. Thus, all-solid-state Z-scheme photocatalytic system attracted some scientific attentions.

Different from the photocatalytic mechanism of the redox-mediator Z-scheme, in the solid-state Z-scheme photocatalytic system, the two semiconductors are directly contacted via conductor as electron mediator (such as Au [260,261], RGO [31,262] and MWCNT [263]) to form Ohmic contacts with low contacting resistance. As shown in Fig. 21(b), both the photogenerated electrons in the CB of PS II and photogenerated holes in the VB of PS I transfer to the electron mediator and recombine with each other, leaving electrons in the CB of PS I and holes in the VB of PS II, which are mostly reserved for the targeting reduction and oxidation reactions, respectively. For the solid-state Z-scheme photocatalytic system, the distance of electrons transfer is reduced due to the presence of electron mediator, therefore reducing the recombine of the photogenerated $e^{-} / h^{+}$pairs. Without the D/A pairs, the solid-state Z-scheme photocatalytic system can work not only in the liquid phase, but also in the gas phase. However, when the electron mediator is noble metal, the development of the solid-state Z-scheme photocatalytic systems are limited by the expensive cost and shielding effect [264]. Table 9 shows the applications of all-solid-state Z-scheme photocatalytic system in hydrogen evolution applications.

In 2016, Ma's group [265] reported sandwich-structured Z-scheme g- $\mathrm{C}_{3} \mathrm{~N}_{4}-\mathrm{Au}-\mathrm{CdZnS}$ nanoparticles system for visible light photocatalytic hydrogen evolution with $24.6 \mathrm{mmol} \cdot \mathrm{h}^{-1} \cdot \mathrm{g}^{-1}$ hydrogen yield. Attribute to the Z-scheme charge-carrier transfer mechanism, Au is used as the electron transfer mediator between g- $\mathrm{C}_{3} \mathrm{~N}_{4}$ and $\mathrm{CdZnS}$, which improves the photocatalytic performance of the composite. Au acts as an electron tran- 
Table 9

Summary of all-solid-state Z-scheme photocatalyst systems for $\mathrm{H}_{2}$ evolution.

\begin{tabular}{|c|c|c|c|c|c|c|c|c|}
\hline Photocatalyst & Mediator & Cocatalyst & Light source & $\begin{array}{c}\text { Weight } \\
\text { (mg) }\end{array}$ & Solution & Activity & $\mathrm{AQY}$ & Year/Ref. \\
\hline $\mathrm{CdS}-\mathrm{Au}_{\mathrm{x}}-\mathrm{ZnO}$ & $\mathrm{Au}$ & $\mathrm{ZnO}$ & 300 W Xe lamp $(\lambda \geq 420 \mathrm{~nm})$ & 20 & $80 \mathrm{~mL} \mathrm{Na}{ }_{2} \mathrm{~S} \mathrm{Na}_{2} \mathrm{SO}_{3}$ & $102.9 \mu \mathrm{mol} \cdot \mathrm{h}^{-1}$ & $\begin{array}{c}9.6 \% \\
(420 \mathrm{~nm})\end{array}$ & 2018 [267] \\
\hline $\begin{array}{l}\mathrm{Cd}_{0.5} \mathrm{Zn}_{0.5} \mathrm{~S} / \mathrm{RGO} / \mathrm{CN}-4 \\
0 \%\end{array}$ & RGO & $\mathrm{Cd}_{0.5} \mathrm{Zn}_{0.5} \mathrm{~S}$ & $300 \mathrm{~W}$ Xe lamp $(\lambda \geq 420 \mathrm{~nm})$ & 30 & $\begin{array}{c}100 \mathrm{~mL} 0.1 \mathrm{M} \\
\mathrm{Na}_{2} \mathrm{~S} / 0.1 \mathrm{M} \mathrm{Na}_{2} \mathrm{SO}_{3}\end{array}$ & $\begin{array}{c}39.24 \\
\mathrm{mmol} \cdot \mathrm{h}^{-1} \cdot \mathrm{g}^{-1}\end{array}$ & $\begin{array}{c}37.88 \% \\
(420 \mathrm{~nm})\end{array}$ & $2018[262]$ \\
\hline $\begin{array}{l}\text { CdS } / 3 \text { wt. } \% \text { Ag/15 } \\
\text { wt. } \% \text { g- } \mathrm{C}_{3} \mathrm{~N}_{4}\end{array}$ & $\mathrm{Ag}$ & $\mathrm{g}-\mathrm{C}_{3} \mathrm{~N}_{4}$ & $300 \mathrm{~W}$ Xe lamp $(\lambda \geq 420 \mathrm{~nm})$ & 50 & $\begin{array}{c}100 \mathrm{~mL} 10 \text { vol\% } \\
\text { TEOA }\end{array}$ & $\begin{array}{c}1376.0 \\
\mu \mathrm{mol} \cdot \mathrm{h}^{-1} \cdot \mathrm{g}^{-1}\end{array}$ & - & $2018[268]$ \\
\hline $\begin{array}{l}\mathrm{Zn}_{0.5} \mathrm{Cd}_{0.5} \mathrm{~S}-\mathrm{MWCNT}- \\
\mathrm{TiO}_{2}\end{array}$ & MWCNT & $\mathrm{Zn}_{0.5} \mathrm{Cd}_{0.5} \mathrm{~S}$ & 500 W Xe lamp & 30 & $\begin{array}{c}100 \mathrm{~mL} 0.1 \mathrm{M} \\
\mathrm{Na}_{2} \mathrm{~S} / 0.1 \mathrm{M} \mathrm{Na}_{2} \mathrm{SO}_{3}\end{array}$ & $21.9 \mu \mathrm{mol} \cdot \mathrm{h}^{-1}$ & - & 2017 [263] \\
\hline $\mathrm{NiTiO}_{3} / \mathrm{Ag} / \mathrm{g}-\mathrm{C}_{3} \mathrm{~N}_{4}$ & $\mathrm{Ag}$ & $\mathrm{NiTiO}_{3}$ & $300 \mathrm{~W}$ Xe lamp & 4 & $40 \mathrm{~mL} 10 \mathrm{vol} \%$ TEOA & $3351 \mu \mathrm{mol} \cdot \mathrm{h}^{-1} \cdot \mathrm{g}^{-1}$ & - & 2019 [269] \\
\hline $\mathrm{Ti}_{3} \mathrm{C}_{2} @ \mathrm{CdS} / \mathrm{MoS}_{2}$ & CdS & $\mathrm{Ti}_{3} \mathrm{C}_{2}$ & $300 \mathrm{~W}$ Xe lamp $(\lambda \geq 420 \mathrm{~nm})$ & 30 & $\begin{array}{c}100 \mathrm{~mL} \mathrm{20 \%} \mathrm{lactic} \\
\text { acid }\end{array}$ & $8.47 \mathrm{mmol} \cdot \mathrm{h}^{-1} \cdot \mathrm{g}^{-1}$ & - & 2019 [270] \\
\hline $\mathrm{g}-\mathrm{C}_{3} \mathrm{~N}_{4} / \mathrm{Au} / \mathrm{CdZnS}$ & $\mathrm{Au}$ & $\mathrm{CdZnS}$ & $150 \mathrm{~W}$ Xe lamp $(\lambda \geq 420 \mathrm{~nm})$ & 100 & $\begin{array}{c}100 \mathrm{~mL} 0.35 \mathrm{M} \\
\mathrm{Na}_{2} \mathrm{~S} / 0.25 \mathrm{M} \mathrm{Na}_{2} \mathrm{SO}_{3}\end{array}$ & $\begin{array}{c}24.6 \\
\mathrm{mmol} \cdot 4 \mathrm{~h}^{-1} \cdot \mathrm{g}^{-1}\end{array}$ & - & 2016 [265] \\
\hline $\mathrm{Cd}_{0.8} \mathrm{Zn}_{0.2} \mathrm{~S} / \mathrm{Au} / \mathrm{g}-\mathrm{C}_{3} \mathrm{~N}_{4}$ & $\mathrm{Au}$ & $\mathrm{Cd}_{0.8} \mathrm{Zn}_{0.2} \mathrm{~S}$ & 300 W Xe lamp $(\lambda \geq 420 \mathrm{~nm})$ & 50 & $100 \mathrm{~mL} 0.1 \mathrm{M}$ glucose & $123 \mu \mathrm{mol} \cdot \mathrm{h}^{-1} \cdot \mathrm{g}^{-1}$ & - & $2017[260]$ \\
\hline $\mathrm{Au} @ \mathrm{CdS} / \mathrm{U}-\mathrm{WO}_{3}$ & $\mathrm{Au}$ & CdS & $300 \mathrm{~W}$ Xe lamp $(\lambda \geq 420 \mathrm{~nm})$ & 20 & $\begin{array}{c}100 \mathrm{~mL} 2.34 \mathrm{~g} \\
\mathrm{Na}_{2} \mathrm{~S} / 3.78 \mathrm{~g} \mathrm{Na}_{2} \mathrm{SO}_{3}\end{array}$ & $1.39 \mathrm{mmol} \cdot \mathrm{h}^{-1} \cdot \mathrm{g}^{-1}$ & $\begin{array}{c}3.45 \% \\
(420 \mathrm{~nm})\end{array}$ & 2015 [271] \\
\hline $\mathrm{CdS}-\mathrm{Au}-\mathrm{HCNS}$ & $\mathrm{Au}$ & CdS & $300 \mathrm{~W}$ Xe lamp $(\lambda \geq 420 \mathrm{~nm})$ & 20 & $\begin{array}{c}100 \mathrm{~mL} 10 \mathrm{vol} \% \\
\text { TEOA }\end{array}$ & $277 \mu \mathrm{mol} \cdot \mathrm{h}^{-1}$ & $\begin{array}{c}8.7 \% \\
(420 \mathrm{~nm})\end{array}$ & 2015 [272] \\
\hline $\mathrm{TiO}_{2} / \mathrm{WO}_{3} @ \mathrm{MoS}_{2}$ & $\mathrm{TiO}_{2}$ & $\mathrm{MoS}_{2}$ & 300 W Xe lamp & 50 & $\begin{array}{c}65 \mathrm{~mL} 38.5 \% \text { methyl } \\
\text { alcohol }\end{array}$ & $\begin{array}{c}273.69 \\
\mu \mathrm{mol} \cdot \mathrm{h}^{-1} \cdot \mathrm{g}^{-1}\end{array}$ & - & 2018 [273] \\
\hline $\mathrm{MoO}_{3} / 1 \mathrm{~T}-\mathrm{MoS}_{2} / \mathrm{g}-\mathrm{C}_{3} \mathrm{~N}_{4}$ & $1 \mathrm{~T}-\mathrm{MoS}_{2}$ & $\mathrm{~g}-\mathrm{C}_{3} \mathrm{~N}_{4}$ & $300 \mathrm{~W}$ Xe lamp $(\lambda \geq 420 \mathrm{~nm})$ & 10 & $20 \mathrm{~mL} 10$ vol $\%$ TEOA & $513.0 \mu \mathrm{mol} \cdot \mathrm{h}^{-1} \cdot \mathrm{g}^{-1}$ & - & 2018 [266] \\
\hline $\mathrm{CdS} / \mathrm{RGO} / \mathrm{g}-\mathrm{C}_{3} \mathrm{~N}_{4}$ & RGO & CdS & $350 \mathrm{~W}$ Xe lamp $(\lambda \geq 400 \mathrm{~nm})$ & 20 & $\begin{array}{c}50 \mathrm{~mL} 10 \% \text { lactic } \\
\text { acid }\end{array}$ & $676.5 \mu \mathrm{mol} \cdot \mathrm{h}^{-1} \cdot \mathrm{g}^{-1}$ & $\begin{array}{c}36.5 \% \\
(400 \mathrm{~nm})\end{array}$ & $2017[31]$ \\
\hline $\mathrm{TiO}_{2} / \mathrm{WO}_{3} / \mathrm{Au}$ & $\mathrm{Au}$ & $\mathrm{TiO}_{2}$ & $300 \mathrm{~W}$ Xe lamp $(\lambda \geq 400 \mathrm{~nm})$ & 50 & $\begin{array}{c}65 \mathrm{~mL} \text { 38.5\% methyl } \\
\text { alcohol }\end{array}$ & $269.63 \mu \mathrm{mol} \cdot \mathrm{h}^{-1}$ & - & $2017[261]$ \\
\hline
\end{tabular}

sition mediator that induces the recombination of photogenerated electrons in CdZnS to the photogenerated holes in $\mathrm{g}-\mathrm{C}_{3} \mathrm{~N}_{4}$. Except noble metals, metal sulfide and oxides with significant conductivity have been employed as electron-mediator between the photocatalysts in Z-scheme system. Wang and his co-worker [266] reported a novel composite photocatalyst by sandwiching metallic 1T-phase $\mathrm{MoS}_{2}$ nanosheets between $\mathrm{MoO}_{3}$ and $\mathrm{g}-\mathrm{C}_{3} \mathrm{~N}_{4}$ for the first time, in which the metallic 1T-phase $\mathrm{MoS}_{2}$ nanosheets act as electron mediator between $\mathrm{MoO}_{3}$ and g- $\mathrm{C}_{3} \mathrm{~N}_{4}$ to construct an all-solid-state Z-scheme photocatalytic system, and the photocatalytic $\mathrm{H}_{2}$ evolution rate reaches to $513.0 \mu \mathrm{mol} \cdot \mathrm{h}^{-1} \cdot \mathrm{g}^{-1}$ under visible light irradiation. In addition, non-metal with significant conductivity such as reduced graphene oxide (RGO) have also been used as electron mediator to enhance the photocatalytic hydrogen evolution. Xue et al. [262] constructed ternary $\mathrm{Cd}_{0.5} \mathrm{Zn}_{0.5} \mathrm{~S} / \mathrm{RGO} / \mathrm{g}-\mathrm{C}_{3} \mathrm{~N}_{4}$ all-solid-state Z-scheme via two-step method and obtained an $\mathrm{H}_{2}$ evolution rate of $39.24 \mathrm{mmol} \cdot \mathrm{h}^{-1} \cdot \mathrm{g}^{-1}$ with an AQE of $37.88 \%$ at $420 \mathrm{~nm}$. The enhanced photocatalytic hydrogen evolution performance can be ascribed to the synergistic effect between $\mathrm{Cd}_{0.5} \mathrm{Zn}_{0.5} \mathrm{~S}$ nanospheres and g- $\mathrm{C}_{3} \mathrm{~N}_{4}$ nanosheets in the existence of RGO, which plays a decisive role in the ternary system by acting as a mediator for charge carriers between the two semiconductors.

\subsection{Direct Z-scheme photocatalyst system}

Although the electron mediators play an important role in transferring electrons between PS I and PS II, there are several adverse effects, such as metal-based electron mediators might affect light absorption, and Au could cause SPR effect, which would have great influence on the activity of the photocatalyst. Hence, mediator-free Z-scheme photocatalyst was constructed in 2013, Yu et al. [47] first proposed the concept of a direct Z-scheme photocatalyst. Although the band structures of direct Z-scheme photocatalyst systems are similar to the Type-II heterostructures, the electron transfer path between the two semiconductors is completely different. As schematically illustrated in Fig. 21(c), the direct Z-scheme photocatalyst system was constituted by two closely contacted semiconductors, the electrons directly transfer across the interface for the CB of PS I to the VB of PS II without any mediator, and then participate in the redox reaction occurred on the surface of photocatalysts. As a result, the direct Z-scheme photocatalyst system has a strong redox ability for driving the photocatalytic reactions and spatially separated reductive and oxidative active sites. The application of direct Z-scheme photocatalyst system for $\mathrm{H}_{2}$ production is summarized in Table 10. Yu's group [274] reported a novel $\mathrm{TiO}_{2} / \mathrm{NiS}$ core-shell direct Z-scheme nanostructure hybrid for photocatalytic $\mathrm{H}_{2}$ production with the rate of 655 $\mu \mathrm{mol} \cdot \mathrm{h}^{-1} \cdot \mathrm{g}^{-1}$. As shown in Fig. 23, when the two semiconductors form an ohmic contact, a built-in electric field is formed at the interface between $\mathrm{TiO}_{2}$ and $\mathrm{NiS}$, the electrons in the $\mathrm{CB}$ of $\mathrm{TiO}_{2}$ will recombine with the holes in the $\mathrm{VB}$ of NiS. The photogenerated electrons in the $\mathrm{CB}$ of NiS would effectively reduce $\mathrm{H}_{2} \mathrm{O}$ to produce $\mathrm{H}_{2}$. Meanwhile, the holes in the $\mathrm{VB}$ of $\mathrm{TiO}_{2}$ oxidize the sacrificial agent of methyl. The $\mathrm{TiO}_{2} / \mathrm{NiS}$ direct Z-scheme could effective separate electron-hole pairs to promote photocatalytic hydrogen production.

To date, Liu's group [275] conducted studies on the $\mathrm{Bi}_{2} \mathrm{WO}_{6} / \mathrm{CdS} \mathrm{Z}$-scheme photocatalyst system and obtained a photocatalytic $\mathrm{H}_{2}$ evolution rate of $1223.364 \mu \mathrm{mol} \cdot \mathrm{h}^{-1}$ with AQY 
Table 10

Summary of direct Z-scheme photocatalyst systems for $\mathrm{H}_{2}$ evolution.

\begin{tabular}{|c|c|c|c|c|c|c|c|}
\hline Photocatalyst & Cocatalyst & Light source & $\begin{array}{l}\text { Weight } \\
\text { (mg) }\end{array}$ & Solution & Activity & $\mathrm{AQY}$ & Year/Ref. \\
\hline $\mathrm{Bi}_{2} \mathrm{WO}_{6} / \mathrm{CdS}$ & CdS & $300 \mathrm{~W}$ Xe lamp $(\lambda \geq 420 \mathrm{~nm})$ & 20 & $100 \mathrm{~mL} \mathrm{25 \% \text {methanol }}$ & $1223.364 \mu \mathrm{mol} \cdot \mathrm{h}^{-1}$ & $1.4 \%(420 \mathrm{~nm})$ & 2019 [275] \\
\hline $\mathrm{TiO}_{2} / \mathrm{CdS}$ & $\mathrm{CdS}$ & 350 W Xe lamp & 50 & 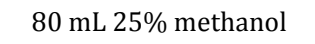 & $51.4 \mu \mathrm{mol} \cdot \mathrm{h}^{-1}$ & - & 2017 [33] \\
\hline $5 \% \mathrm{WO}_{3} / \mathrm{CdS}$-DETA & $\mathrm{WO}_{3}$ & $300 \mathrm{~W}$ Xe lamp $(\lambda \geq 400 \mathrm{~nm})$ & 50 & $\begin{array}{c}100 \mathrm{~mL} 0.35 \mathrm{M} \mathrm{Na}_{2} \mathrm{~S} / \\
0.25 \mathrm{M} \mathrm{Na}_{2} \mathrm{SO}_{3}\end{array}$ & $15522 \mu \mathrm{mol} \cdot \mathrm{h}^{-1} \cdot \mathrm{g}^{-1}$ & - & $2018[35]$ \\
\hline $0.5 \% \mathrm{CdS} / \mathrm{CdWO}_{4}$ & $\mathrm{CdS}$ & 300 W Xe lamp & 10 & $\begin{array}{c}100 \mathrm{~mL} 0.35 \mathrm{M} \mathrm{Na}_{2} \mathrm{~S} / \\
0.25 \mathrm{M} \mathrm{Na}_{2} \mathrm{SO}_{3}\end{array}$ & $2400 \mu \mathrm{mol} \cdot \mathrm{h}^{-1} \cdot \mathrm{g}^{-1}$ & - & $2018[34]$ \\
\hline anatase/rutile $\mathrm{TiO}_{2}$ & $\mathrm{Pt}$ & $350 \mathrm{~W}$ Xe lamp $(\lambda \geq 365 \mathrm{~nm})$ & 50 & 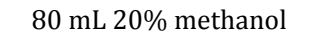 & $324 \mu \mathrm{mol} \cdot \mathrm{h}^{-1}$ & $20.9 \%(365 \mathrm{~nm})$ & 2014 [280] \\
\hline $\mathrm{BCN}-\mathrm{TiO}_{2}$ & $\mathrm{Pt}$ & $300 \mathrm{~W}$ Xe lamp $(\lambda \geq 420 \mathrm{~nm})$ & 50 & $100 \mathrm{~mL} 20$ vol\% TEOA & $19.7 \mu \mathrm{mol} \cdot \mathrm{h}^{-1} \cdot \mathrm{g}^{-1}$ & - & 2017 [281] \\
\hline 10 wt. $\% \mathrm{WO}_{3} / \mathrm{g}-\mathrm{C}_{3} \mathrm{~N}_{4}$ & $\mathrm{WO}_{3}$ & $300 \mathrm{~W}$ Xe lamp $(\lambda \geq 420 \mathrm{~nm})$ & 100 & $100 \mathrm{~mL} 10$ vol\% TEOA & $1853 \mu \mathrm{mol} \cdot \mathrm{h}^{-1} \cdot \mathrm{g}^{-1}$ & - & 2018 [282] \\
\hline $15 \% \mathrm{WO}_{3} / \mathrm{g}-\mathrm{C}_{3} \mathrm{~N}_{4}$ & $\mathrm{WO}_{3}$ & $350 \mathrm{~W}$ Xe lamp & 50 & 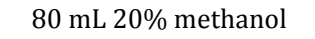 & $982 \mu \mathrm{mol} \cdot \mathrm{h}^{-1} \cdot \mathrm{g}^{-1}$ & - & $2019[14]$ \\
\hline $\mathrm{C}_{3} \mathrm{~N}_{4} / \mathrm{W}_{18} \mathrm{O}_{49}$ & $\mathrm{~W}_{18} \mathrm{O}_{49}$ & $300 \mathrm{~W}$ Xe lamp $(\lambda \geq 400 \mathrm{~nm})$ & 50 & $120 \mathrm{~mL} 10$ vol\% TEOA & $8597 \mu \mathrm{mol} \cdot \mathrm{h}^{-1} \cdot \mathrm{g}^{-1}$ & $39.1 \%(420 \mathrm{~nm})$ & 2017 [283] \\
\hline $\mathrm{WO}_{3} / \mathrm{C}_{3} \mathrm{~N}_{4}$ & $\mathrm{Ni}(\mathrm{OH})_{x}$ & $300 \mathrm{~W}$ Xe lamp $(\lambda \geq 400 \mathrm{~nm})$ & 50 & $80 \mathrm{~mL} 15$ vol\% TEOA & $576 \mu \mathrm{mol} \cdot \mathrm{h}^{-1} \cdot \mathrm{g}^{-1}$ & - & $2017[284]$ \\
\hline $\mathrm{g}-\mathrm{C}_{3} \mathrm{~N}_{4} / \mathrm{WO}_{3}$ & Pt & $300 \mathrm{~W}$ Xe lamp & 50 & $80 \mathrm{~mL} 10$ vol\% TEOA & $3.12 \mathrm{mmol} \cdot \mathrm{h}^{-1} \cdot \mathrm{g}^{-1}$ & - & 2017 [285] \\
\hline $\begin{array}{l}\text { g- } \mathrm{C}_{3} \mathrm{~N}_{4} / \mathrm{Bi}_{4} \mathrm{NbO}_{8} \mathrm{Cl} \\
\text { (molar ratio } 3: 1 \text { ) }\end{array}$ & Pt & $300 \mathrm{~W}$ Xe lamp $(\lambda \geq 420 \mathrm{~nm})$ & 100 & $50 \mathrm{~mL} 10$ vol\% lactic acid & $287.71 \mu \mathrm{mol} \cdot \mathrm{h}^{-1} \cdot \mathrm{g}^{-1}$ & $2.02 \%(420 \mathrm{~nm})$ & 2018 [286] \\
\hline $\mathrm{TiO}_{2} / \mathrm{NiS}$ & $\mathrm{NiS}$ & 350 W Xe lamp & 50 & $80 \mathrm{~mL} \mathrm{20 \%}$ methanol & $655 \mu \mathrm{mol} \cdot \mathrm{h}^{-1} \cdot \mathrm{g}^{-1}$ & - & 2018 [274] \\
\hline $\begin{array}{l}1 \% \mathrm{Bi}_{2} \mathrm{MoO}_{6} / \\
\text { CdSe-DETA }\end{array}$ & Pt & $300 \mathrm{~W}$ Xe lamp $(\lambda \geq 420 \mathrm{~nm})$ & 50 & $\begin{array}{c}100 \mathrm{~mL} 0.35 \mathrm{M} \mathrm{Na}_{2} \mathrm{~S} / \\
0.25 \mathrm{M} \mathrm{Na}_{2} \mathrm{SO}_{3}\end{array}$ & $5.92 \mathrm{mmol} \cdot \mathrm{h}^{-1} \cdot \mathrm{g}^{-1}$ & $27.3 \%(420 \mathrm{~nm})$ & 2019 [278] \\
\hline 20 wt. $\% \mathrm{CdS} / \mathrm{WO}_{3}$ & $\mathrm{Pt}$ & $500 \mathrm{~W}$ Xe lamp $(\lambda \geq 400 \mathrm{~nm})$ & 50 & lactic acid & $2900 \mu \mathrm{mol} \cdot \mathrm{h}^{-1} \cdot \mathrm{g}^{-1}$ & - & 2014 [277] \\
\hline $\mathrm{Zn}_{0.5} \mathrm{Cd}_{0.5} \mathrm{~S} / \mathrm{ZnO}$ & $\mathrm{Zn}_{0.5} \mathrm{Cd}_{0.5} \mathrm{~S}$ & $300 \mathrm{~W}$ Xe lamp & 20 & $\begin{array}{c}100 \mathrm{~mL} 0.75 \mathrm{M} \mathrm{Na}_{2} \mathrm{~S} / \\
1.05 \mathrm{M} \mathrm{Na}_{2} \mathrm{SO}_{3}\end{array}$ & $28.6 \mathrm{mmol} \cdot \mathrm{h}^{-1} \cdot \mathrm{g}^{-1}$ & - & 2018 [287] \\
\hline $\mathrm{WO}_{3-x} / \mathrm{Zn}_{0.3} \mathrm{Cd}_{0.7} \mathrm{~S}$ & $\mathrm{Zn}_{0.3} \mathrm{Cd}_{0.7} \mathrm{~S}$ & $300 \mathrm{~W}$ Xe lamp $(\lambda \geq 420 \mathrm{~nm})$ & 100 & $\begin{array}{c}100 \mathrm{~mL} 0.1 \mathrm{M} \mathrm{Na}_{2} \mathrm{~S} / \\
0.1 \mathrm{M} \mathrm{Na}_{2} \mathrm{SO}_{3}\end{array}$ & $352.1 \mu \mathrm{mol} \cdot \mathrm{h}^{-1}$ & $7.3 \%(420 \mathrm{~nm})$ & 2017 [288] \\
\hline g- $\mathrm{C}_{3} \mathrm{~N}_{4} / \mathrm{Ag}_{2} \mathrm{CrO}_{4}$ & $\mathrm{Pt}$ & $300 \mathrm{~W}$ Xe lamp $(\lambda \geq 420 \mathrm{~nm})$ & 50 & $\begin{array}{l}100 \mathrm{~mL} 25 \% \text { metha- } \\
\text { nol } / 10 \mathrm{mM} \mathrm{NaHCO}\end{array}$ & $902.1 \mu \mathrm{mol} \cdot \mathrm{h}^{-1} \cdot \mathrm{g}^{-1}$ & $23.1 \%(420 \mathrm{~nm})$ & 2018 [279] \\
\hline 10 wt. $\% \mathrm{WO}_{3} / \mathrm{g}-\mathrm{C}_{3} \mathrm{~N}_{4}$ & $\mathrm{Pt}$ & $300 \mathrm{~W}$ Xe lamp $(\lambda \geq 420 \mathrm{~nm})$ & 40 & $40 \mathrm{~mL} 10$ vol\% TEOA & $110 \mu \mathrm{mol} \cdot \mathrm{h}^{-1} \cdot \mathrm{g}^{-1}$ & - & 2014 [289] \\
\hline $\mathrm{CdS} / \mathrm{ZnO}$ & Pt & 225 W Xe lamp & 10 & $\begin{array}{c}25 \mathrm{~mL} 0.75 \mathrm{M} \mathrm{Na}_{2} \mathrm{~S} / \\
1.05 \mathrm{M} \mathrm{Na}_{2} \mathrm{SO}_{3}\end{array}$ & $22.12 \mathrm{mmol} \cdot \mathrm{h}^{-1} \cdot \mathrm{g}^{-1}$ & - & 2017 [276] \\
\hline
\end{tabular}

of $1.4 \%$ at $420 \mathrm{~nm}$. The $\mathrm{H}_{2}$ production rate was significantly higher than the one produced by using pure $\mathrm{Bi}_{2} \mathrm{WO}_{6}$ or $\mathrm{CdS}$ photocatalysts (Fig. 24). The excellent photocatalytic performance is due to the intrinsic properties of the direct Z-scheme $\mathrm{Bi}_{2} \mathrm{WO}_{6} / \mathrm{CdS}$ photocatalyst system, which replaces the electron mediator with an ohmic contact to shorten the distance of electron transfer, and thus improves the separation of electrons and holes. Pt acts as dual cocatalyst to improve the $\mathrm{H}_{2}$ evolution activity in the photocatalyst system, because of the enhanced charge separation of photogenerated charge carriers and the efficient photocatalytic $\mathrm{H}_{2}$ production. $\mathrm{Pt}$ is also applicable to other direct Z-scheme systems and acts as a cocatalyst to promote the activity of photocatalytic $\mathrm{H}_{2}$ [276-279].
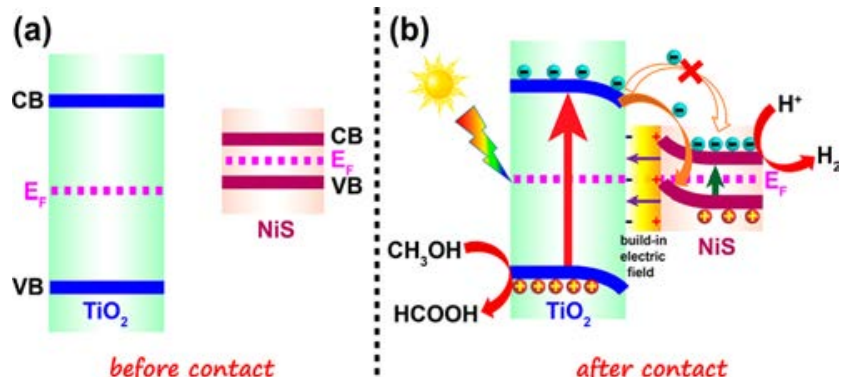

Fig. 23. Schematic illustration of $\mathrm{TiO}_{2}$ and NiS direct Z-scheme heterojunction (a) before and (b) after contact along with the charge transfer and separation in TN10 under UV-visible light irradiation. From Ref. [274].

\section{MOFs cocatalysts for photocatalytic $\mathrm{H}_{2}$ evolution}

Metal organic frameworks (MOFs) are well known as porous materials consisting of metal nodes and organic ligands with unique catalytic natures [290,291]. MOFs have been often used as electrocatalysts and photocatalysts because of their high design flexibility, large specific surface area and tunable porous channels, which are available for exposing numerous active sites to drive the redox reactions and shorten the transfer distance of photoexcited carriers to the pore surface [292]. In addition, MOF-derived materials have been found to serve as precursors for porous semiconductor materials, which includes metal phosphide, metal oxides, carbon materials and metal sulfides via facile thermal treatment, phosphorization and sulfidation process [293-296]. The as-prepared MOF-based materials can not only maintain the initial structural features of MOF materials that ensures the mono-dispersion of metals centers, but also improve the conductivity property of the materials. Thus, tremendous efforts have been made to explore MOF-derived materials in photocatalytic water splitting.

MOF-based materials as cocatalysts in the hydrogen evolution half-reaction system can serve various metal ions, and facilitate the doping of $\mathrm{C}$ and $\mathrm{N}$ to the MOF-derived semiconductors, and more important they can provide high surface areas to expose numerous active sites.

Ui0-66 as a representative stable MOF owns intrinsic photocatalytic $\mathrm{H}_{2}$ evolution activity. Jiang's group [297] has syn- 

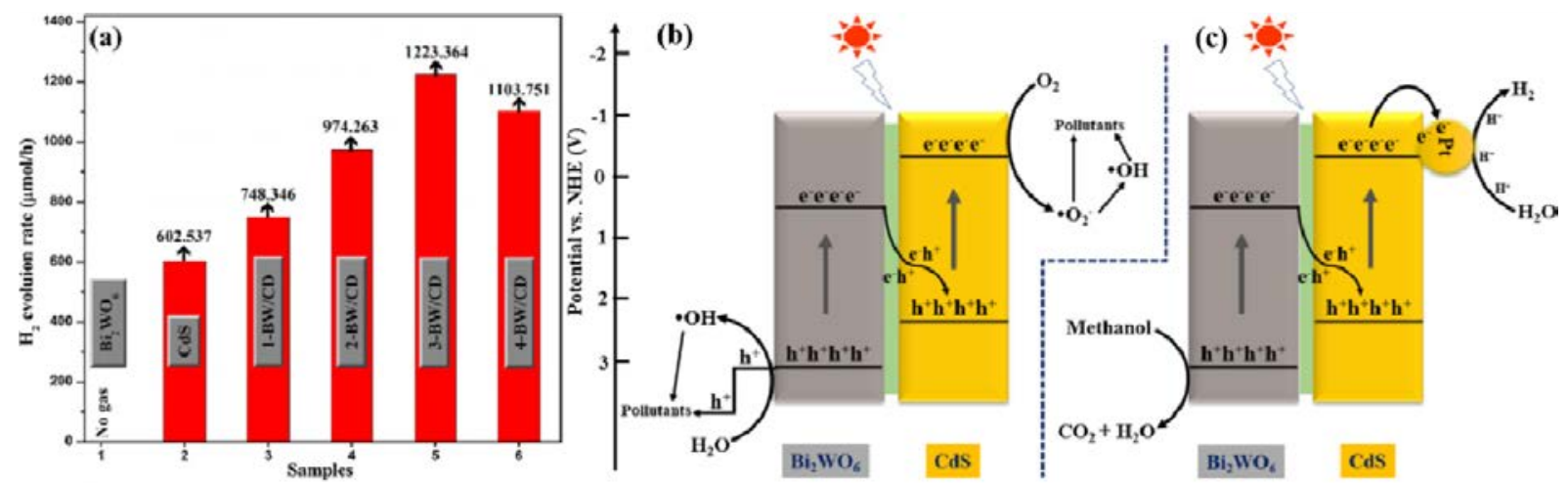

Fig. 24. (a) Comparison of photocatalytic $\mathrm{H}_{2}$ evolution over $\mathrm{Bi}_{2} \mathrm{WO}_{6}$, pristine $\mathrm{CdS}$ nanowires and $\mathrm{Bi}_{2} \mathrm{WO}_{6} / \mathrm{CdS}(1-\mathrm{BW} / \mathrm{CD}, 2-\mathrm{BW} / \mathrm{CD}, 3-\mathrm{BW} / \mathrm{CD}$ and 4-BW/CD) from aqueous solution containing $25 \%$ methanol by volume; (b) and (c) Schematic photocatalytic decomposition and photocatalytic $\mathrm{H}_{2}$ evolution mechanism over $\mathrm{Bi}_{2} \mathrm{WO}_{6} / \mathrm{CdS}$ in Z-scheme photocatalytic system under visible-light illumination. From Ref. [275].

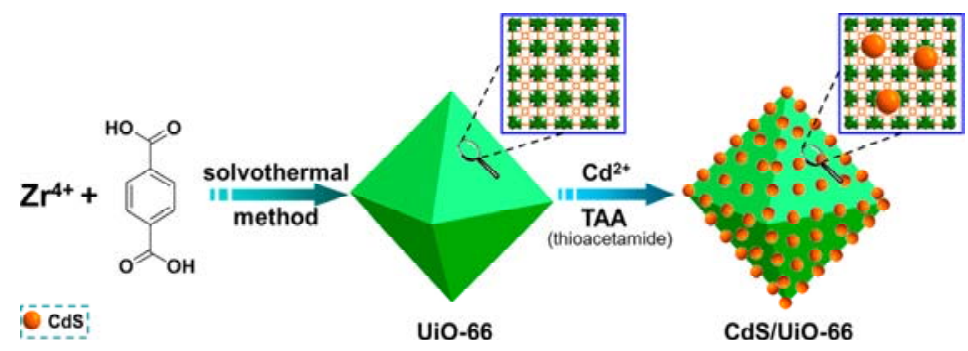

Fig. 25. Synthetic Process for CdS/UiO-66 Composites. From Ref. [297]

thesized the composite of CdS-decorated UiO-66 that not only has taken the advantage of both CdS and MOF, but also avoid the drawbacks of each component. CdS/UiO-66 (Fig. 25) exhibits high $\mathrm{H}_{2}$ production activity for water splitting of $\mathrm{H}_{2}$ evolution under visible light irradiation, the key contribution of the composite is that CdS has the capability to adsorb visible light and UiO-66 provides a large surface area to disperse the CdS on the MOF surface, so that providing more adsorption sites and photocatalytic reaction centers. ZnIn2 $\mathrm{S}_{4} @ \mathrm{NH}_{2}-\mathrm{MIL}-125$ (Ti) nanocomposites were obtained by a simple solvothermal method and $\mathrm{ZnIn}_{2} \mathrm{~S}_{4}$ nanosheets were also highly dispersed on the surface of $\mathrm{NH}_{2}-\mathrm{MIL}-125$ (Ti) MOF [298]. The composite displayed higher photocatalytic performance towards $\mathrm{H}_{2}$ production and process stability during the photocatalytic reactions under visible light irradiation. In addition, the g- $\mathrm{C}_{3} \mathrm{~N}_{4} / \mathrm{MOF}$ (Fig. 26) heterojunctions that were synthesized by combined MOFs (UiO-66) also displayed outstanding photocatalytic performance toward $\mathrm{H}_{2}$ production, as well as $\mathrm{CO}_{2}$ reduction and organic pollutants degradation [299]. Interestingly, crystalline and porous covalent organic frameworks (COFs) and metal-organic frameworks (MOFs) materials are amazing combinations in the field of photocatalytic $\mathrm{H}_{2}$ evolution. Zhang et al. [300] have successfully synthesized MOF/COF (Fig. 27) hybrid materials with high surface area, porous framework and high crystallinity by integrating COF (TpPa-1-COF) with stable MOF $\left(\mathrm{NH}_{2}\right.$-UiO-66). Especially, the resulting $\mathrm{NH}_{2}$-UiO-66/TpPa-1-COF (4:6) exhibits excellent photocatalytic $\mathrm{H}_{2}$ production rate of $23.41 \mathrm{mmol} \cdot \mathrm{g}^{-1} \cdot \mathrm{h}^{-1}$. This design will provide a new path to construct efficient photocatalysts for hydrogen evolution.

MOF-derived metal sulfides can also be well used as cocata- lysts for photocatalysts. Kumar et al. [301] have incorporated MOF-derived hollow structure of $\mathrm{Co}_{4} \mathrm{~S}_{3}$ with CdS particles (Fig.

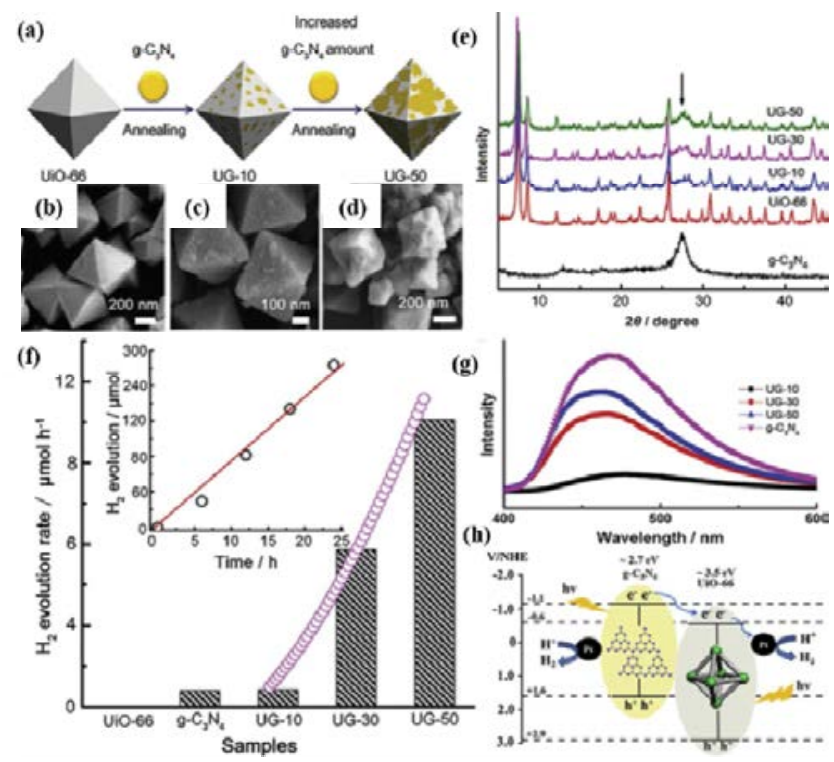

Fig. 26. (a) Schematic illustration of decorating g- $\mathrm{C}_{3} \mathrm{~N}_{4}$ on UiO-66 octahedrons via annealing process. Increasing the g- $\mathrm{C}_{3} \mathrm{~N}_{4}$ amount leads to higher coverage of g- $\mathrm{C}_{3} \mathrm{~N}_{4}$ on UiO-66 octahedrons. SEM images of (b) UiO-66, (c) UG-10 and (d) UG-50. (e) XRD patterns of g- $\mathrm{C}_{3} \mathrm{~N}_{4}, \mathrm{UiO}-66$, UG-10, UG-30 and UG-50 hybrid photocatalysts. (f) The histogram showing the $\mathrm{H}_{2}$ production rate over UG- $x$ samples $(x=10,30$ and 50). The $\mathrm{H}_{2}$ production rate was taken from the first 4 h photocatalytic reaction. The $\mathrm{H}_{2}$ production rate over pristine UiO-66 and $\mathrm{g}-\mathrm{C}_{3} \mathrm{~N}_{4}$ was also included for comparison. The inset shows the long term $\mathrm{H}_{2}$ production over UG-50 sample. (g) The stable state PL spectra of UG- $x(x=10,30$ and 50). (h) The schematic illustration of possible mechanism. From Ref. [299]. 

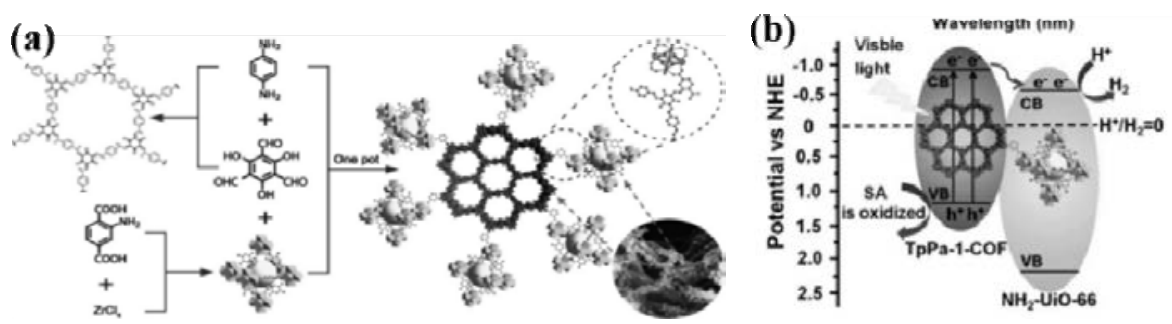

Fig. 27. (a) Schematic illustration of the synthesis of $\mathrm{NH}_{2}-\mathrm{UiO}-66 / \mathrm{TpPa}-1-\mathrm{COF}$ hybrid material; (b) Mechanism schematic of $\mathrm{NH}$-UiO-66/TpPa-1-COF (4:6) hybrid material. From Ref. [300].

28) via a facile wet chemical method. The optimized $\mathrm{Co}_{4} \mathrm{~S}_{3} / \mathrm{CdS}$ composites exhibited superior photocatalytic performance and the $\mathrm{H}_{2}$ evolution rate is $12,360 \mu \mathrm{mol} \cdot \mathrm{h}^{-1} \cdot \mathrm{g}^{-1}$. The high catalytic performance can be ascribed to the abundant active sites and onion slice-type hollow structure in MOF-derived $\mathrm{Co}_{4} \mathrm{~S}_{3}$, which were beneficial for effective separation and transfer of photo-induced charge carriers and boosting the surface shuttling properties.

Metal phosphide has been considered to be the most interesting co-catalysts with unique charging nature (positive charged in metal and negative charged in phosphorous [302,303] for water splitting, especially for HER. Xu et al. [304] have constructed $\mathrm{Fe}_{2} \mathrm{P} / \mathrm{Ni}_{2} \mathrm{P}$-modified g- $\mathrm{C}_{3} \mathrm{~N}_{4}$ photocatalysts, in which the $\mathrm{C}$, $\mathrm{N}$ co-doped $\mathrm{Fe}_{2} \mathrm{P} / \mathrm{Ni}_{2} \mathrm{P}$ cocatalyst was derived from $\mathrm{NH}_{2}$-MIL-101(Fe)/Ni(OH)2. The g- $\mathrm{C}_{3} \mathrm{~N}_{4}$ nanosheets were deposited on the surface of $\mathrm{Ni}(\mathrm{OH})_{2}$ anchored $\mathrm{NH}_{2}-\mathrm{MIL}-101(\mathrm{Fe})$ MOF matrix and then were phosphidized. The photocatalyst showed excellent $\mathrm{H}_{2}$ evolution rate of $13.81 \mathrm{mmol} \cdot \mathrm{h}^{-1} \cdot \mathrm{g}^{-1}$, which was contributed to the hollow polyhedrons structure that facilitated the efficient charges separation and transfer between the interface of $\mathrm{g}-\mathrm{C}_{3} \mathrm{~N}_{4}, \mathrm{CN} / \mathrm{Fe}_{2} \mathrm{P}$ and $\mathrm{Ni}_{2} \mathrm{P}$. Kumar et al. [305] also utilized nickel phosphide $\left(\mathrm{Ni}_{2} \mathrm{P}\right)$ as cocatalysts to modify CdS, which is derived from metal-organic framework (Ni-MOF) (Fig. 29). The optimized composite material exhibited better photocatalytic activity than Pt loaded CdS.

Metal oxide nanostructures are considered to be the most important photocatalysts due to its low cost, low toxicity and high stability. Lin's group [20] have developed a system of $\mathrm{Fe}_{2} \mathrm{O}_{3} @ \mathrm{TiO}_{2}$ (Fig. 30) with MIL-101(Fe) as template and Fe precursor and then calcined in air to decompose the core. This material shows interesting photophysical performance due to its ability to split water under visible light irradiation. In addition, the MOF templating synthesizprocedure can be well modulated by modified varying the MOF and coating materials. Yao's group [306] also incorporated small amounts of uniform $\mathrm{GO}$ in MOF templated iron oxide $\left(\alpha-\mathrm{Fe}_{2} \mathrm{O}_{3}\right.$ and $\gamma$ - $\left.\mathrm{Fe}_{2} \mathrm{O}_{3}\right)$, the heterojunction resulted in highly promoted photo-induced charge separation and transportation for synergistic effect between the reduced graphene and iron oxide. As a consequence, the as-prepared maghemite-carbonaceous composite showed

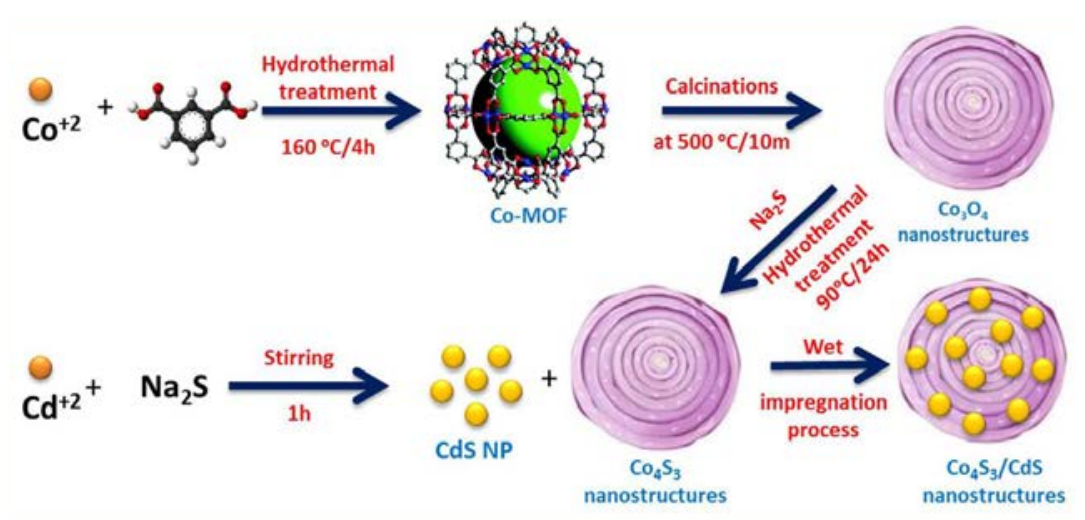

Fig. 28. Schematic representation of material preparation: synthesis of Co-MOFs, synthesis of cobalt sulfide derived from Co-metal-organic framework and synthesis of $\mathrm{Co}_{4} \mathrm{~S}_{3} / \mathrm{CdS}$ composite. From Ref. [301].

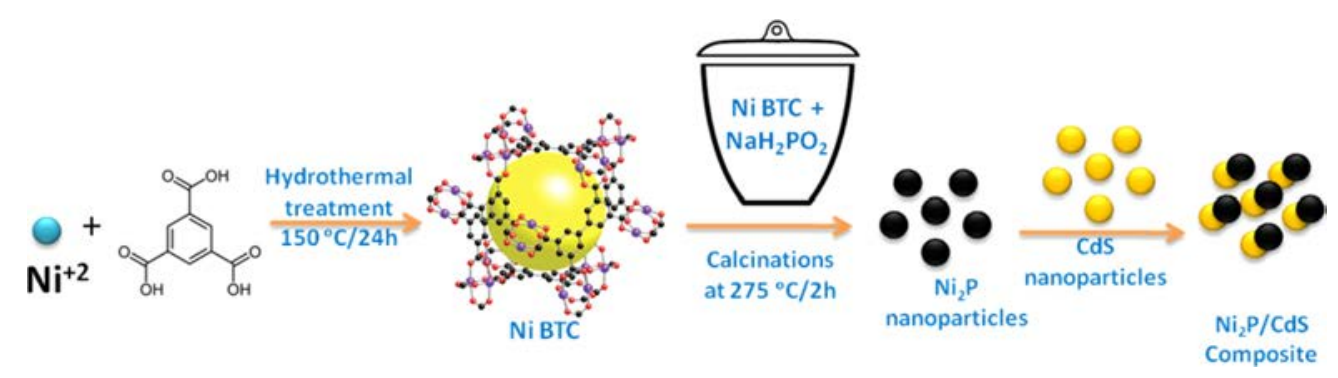

Fig. 29. Synthesis of Ni-BTC MOFs, nickel phosphides derived from Ni-BTC metal-organic frameworks, and $\mathrm{Ni}_{2} \mathrm{P} / \mathrm{CdS}$ composite. From Ref. [305]. 
Table 11

Summary of MOF-based cocatalysts for photocatalytic HER in recent publications.

\begin{tabular}{|c|c|c|c|c|c|}
\hline Photocatalyst & Irradiation & Solvent & $\mathrm{H}_{2}$ evolution rate & Stability test & Ref. \\
\hline CdS/MIL-101(Cr) & Visible-light & Lactic acid & $1.1 \mathrm{mmol} \cdot \mathrm{h}^{-1} \cdot \mathrm{g}^{-1}$ & $5 \mathrm{~h}$ & [308] \\
\hline $1 \mathrm{TMoS}_{2} / \mathrm{MIL}-125-\mathrm{NH}_{2}$ & UV-Vis light & N/A & $2.094 \mathrm{mmol} \cdot \mathrm{h}^{-1} \cdot \mathrm{g}^{-1}$ & $72 \mathrm{~h}$ & [309] \\
\hline g- $\mathrm{C}_{3} \mathrm{~N}_{4} / \mathrm{UiO}-66 / \mathrm{Ni}_{2} \mathrm{P}$ & 5 W LED white light & TEOA & $0.2 \mathrm{mmol} \cdot 5 \mathrm{~h}^{-1} \cdot \mathrm{g}^{-1}$ & $20 \mathrm{~h}$ & [312] \\
\hline $\mathrm{Ni}_{2} \mathrm{P} / \mathrm{CdS}$ & Simulated solar & Lactic acid & $33.48 \mathrm{mmol} \cdot \mathrm{h}^{-1} \cdot \mathrm{g}^{-1}$ & $25 \mathrm{~h}$ & [305] \\
\hline $\mathrm{MoS}_{2} @ \mathrm{TiO}_{2}$ & UV-Vis light & TEOA & $10.046 \mathrm{mmol} \cdot \mathrm{h}^{-1} \cdot \mathrm{g}^{-1}$ & $30 \mathrm{~h}$ & {$[310]$} \\
\hline $\mathrm{Cu}_{2} \mathrm{O} @ \mathrm{C}_{3} \mathrm{~N}$ & UV-Vis light & EDTA & $0.018 \mathrm{mmol} \cdot 27 \mathrm{~h}^{-1} \cdot \mathrm{g}^{-1}$ & $\mathrm{~N} / \mathrm{A}$ & [311] \\
\hline $\mathrm{Co}_{3} \mathrm{O}_{4} / \mathrm{TiO}_{2}$ & UV-Vis light & SED & $7 \mathrm{mmol} \cdot \mathrm{h}^{-1} \cdot \mathrm{g}^{-1}$ & N/A & [313] \\
\hline $\mathrm{MoS}_{2}-\mathrm{ZnS}$ & UV-Vis light & TEOA & $0.411 \mathrm{mmol} \cdot \mathrm{h}^{-1} \cdot \mathrm{g}^{-1}$ & N/A & [314] \\
\hline $\mathrm{Ni}_{x} \mathrm{Mo}_{1-\mathrm{x}} \mathrm{S}_{2} / \mathrm{MOF}-5 @ \mathrm{~g}-\mathrm{C}_{3} \mathrm{~N}_{4}$ & UV-Vis light & TEOA & $0.319 \mathrm{mmol} \cdot \mathrm{h}^{-1} \cdot \mathrm{g}^{-1}$ & $5 \mathrm{~h}$ & [315] \\
\hline $\mathrm{Co}_{4} \mathrm{~S}_{3} / \mathrm{CdS}$ & UV-Vis light & Lactic acid & $12.36 \mathrm{mmol} \cdot \mathrm{h}^{-1} \cdot \mathrm{g}^{-1}$ & $65 \mathrm{~h}$ & [301] \\
\hline
\end{tabular}

an ideal photocatalytic $\mathrm{H}_{2}$ evolution rate of $318 \mu \mathrm{mol} \cdot \mathrm{h}^{-1} \cdot \mathrm{g}^{-1}$.

Porous Pt-doped heterojunction, Pt-ZnO- $\mathrm{Co}_{3} \mathrm{O}_{4}, \mathrm{Pt}-\mathrm{ZnS}-\mathrm{CoS}^{\prime}$ and Pt-Zn $\mathrm{P}_{2}$-CoP (Fig. 31) can be obtained via facile oxidation, sulfurization and phosphorization treatment of ZnCo-zeolitic-imidazolate-framework (ZnCo-ZIF) and then doped Pt nanoparticles. The composite exhibits excellent photocatalytic performance towards $\mathrm{H}_{2}$ production by water splitting, which is attributed to the porous structures of the MOF (a)

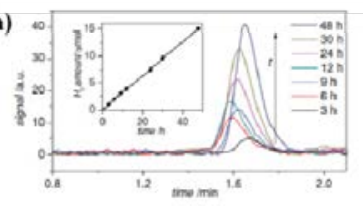

(b)

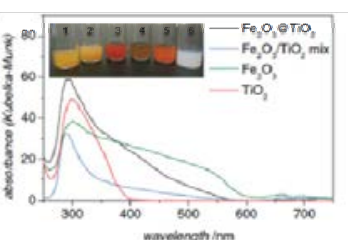

(c)

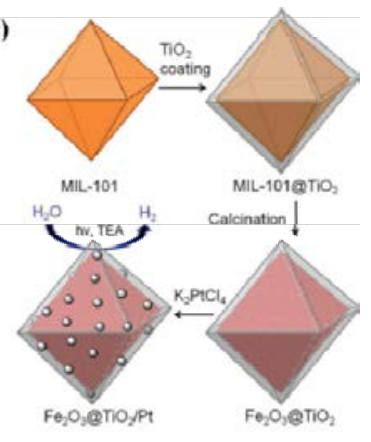

Fig. 30. (a) $\mathrm{H}_{2}$ produced by $\mathrm{Fe}_{2} \mathrm{O}_{3} @ \mathrm{TiO}_{2}$ in $20 / 1 v / v \mathrm{H}_{2} \mathrm{O} / \mathrm{TEA}$ at various time points over $48 \mathrm{~h}$, with a $420 \mathrm{~nm}$ filter. The inset shows the amount of $\mathrm{H}_{2}$ produced over this time period. (b) UV-vis diffuse reflectance spectra of $\mathrm{Fe}_{2} \mathrm{O}_{3} @ \mathrm{TiO}_{2}$, a physical mixture of $\mathrm{Fe}_{2} \mathrm{O}_{3}$ and $\mathrm{TiO}_{2}, \mathrm{Fe}_{2} \mathrm{O}_{3}$ alone, and $\mathrm{TiO}_{2}$ alone. The inset shows a photograph of dispersions of MIL-101 (1), MIL-101@ $\mathrm{TiO}_{2}$ (2), $\mathrm{Fe}_{2} \mathrm{O}_{3} @ \mathrm{TiO}_{2}$ (3), $\mathrm{Fe}_{2} \mathrm{O}_{3} @ \mathrm{TiO}_{2} / \mathrm{Pt}(4)$, $\mathrm{Fe}_{2} \mathrm{O}_{3}$ (5), and $\mathrm{TiO}_{2}$ (6) in ethanol. (c) MOF-templated synthesis of $\mathrm{Fe}_{2} \mathrm{O}_{3} @ \mathrm{TiO}_{2}$ by coating MIL-101 with $\mathrm{TiO}_{2}$ followed by calcination, and its use for photocatalytic hydrogen production after depositing Pt particles. From Ref. [20].

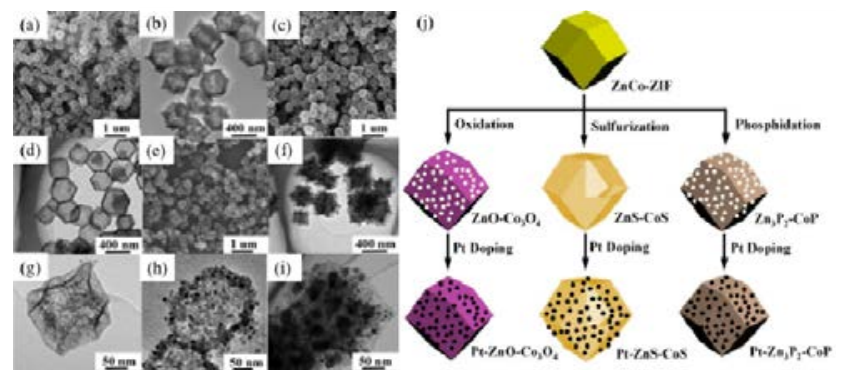

Fig. 31. (a) SEM and (b) TEM images of $\mathrm{ZnO}-\mathrm{Co}_{3} \mathrm{O}_{4}$; (c) $\mathrm{SEM}$ and (d) TEM images of ZnS-CoS; (e) SEM and (f) TEM images of $\mathrm{Zn}_{3} \mathrm{P}_{2}-\mathrm{CoP}$; TEM images of (g) Pt-ZnO-Co $\mathrm{O}_{4}$, (h) Pt-ZnS-CoS, and (i) Pt-Zn ${ }_{3} \mathrm{P}_{2}-\mathrm{CoP}$. (j) The schematic illustration of the fabrication of Pt-ZnO- $\mathrm{Co}_{3} \mathrm{O}_{4}, \mathrm{Pt}-\mathrm{ZnS}-\mathrm{CoS}$, and $\mathrm{Pt}-\mathrm{Zn}_{3} \mathrm{P}_{2}$-CoP photocatalysts. From Ref. [307]. materials that can significantly enhance the light adsorption and afford abundant active sites. In addition, suitable band matching in heterojunctions facilitates the efficient photo-induced charge separation and transportation [307].

Apart from the abovementioned works, Table 11 summarizes other recently published MOF-based photocatalysts for photocatalytic HER, including pristine MOF-based materials, CdS/MIL-101(Cr) [308], $1 \mathrm{TMoS}_{2} / \mathrm{MIL}-125-\mathrm{NH}_{2}$ [309], MOF-derived materials, $\mathrm{MoS}_{2} @ \mathrm{TiO}_{2}$ [310], $\mathrm{Cu}_{2} \mathrm{O} @ \mathrm{C}_{3} \mathrm{~N}$ [311], etc.

\section{Summary and outlook}

In the past decade, semiconductor-based photocatalytic hydrogen production has grown tremendously to be more efficient in converting solar energy into chemical energy. A high performance photocatalytic $\mathrm{H}_{2}$ evolution reaction generally requires high charge separation and effectually charge transportation, which are necessary for loading cocatalysts. In this review, we summarized the research progress on several cocatalysts for photocatalytic hydrogen production in the past decade. It has been proved that integrating these cocatalysts with semiconductors to construct specific structures can significantly improve the photocatalytic activity for $\mathrm{H}_{2}$ evolution. For comparison, TableS 2-11 summarize the latest developments in photocatalytic $\mathrm{H}_{2}$ evolution over various kinds of cocatalysts including single, dual, Z-scheme and MOFs.

The cocatalysts have been extensively investigated in photocatalytic $\mathrm{H}_{2}$ evolution to rapidly trap electrons from the bulk of the semiconductors, which results in efficienct separation of charge carries and suppressed recombination of $e^{-}-h^{+}$pairs. Additionally, the cocatalysts could supply active sites for water reduction and reduce the surface activation energy to catalyze the surface chemical reactions. Further, the cocatalysts usually possess good electrical conductivity, which could boost efficienct transfer of charge carriers.

At present, the cocatalysts used in photocatalytic hydrogen evolution systems has evolved from noble-metals to transition metals and alloys and from single systems to complex systems to enhance the production of $\mathrm{H}_{2}$ evolution. In the above transitions, researchers have achieved a great progress in the field of photocatalytic hydrogen production via water spilling. However, the matching relationship between cocatalysts and photo- 


\section{Graphical Abstract}

Chin. J. Catal., 2020, 41: 642-671 doi: S1872-2067(19)63469-8

The roles and mechanism of cocatalysts in photocatalytic water splitting to produce hydrogen

Nan Xiao, Songsong Li, Xuli Li, Lei Ge*, Yangqin Gao, Ning Li China University of Petroleum

The roles and mechanism of cocatalysts, extended light harvest, enhanced charge carrier separation efficiency and improved stability are summarized to offer a conceptual basis for designing and developing highly efficient photocatalytic $\mathrm{H}_{2}$-evolution cocatalysts.

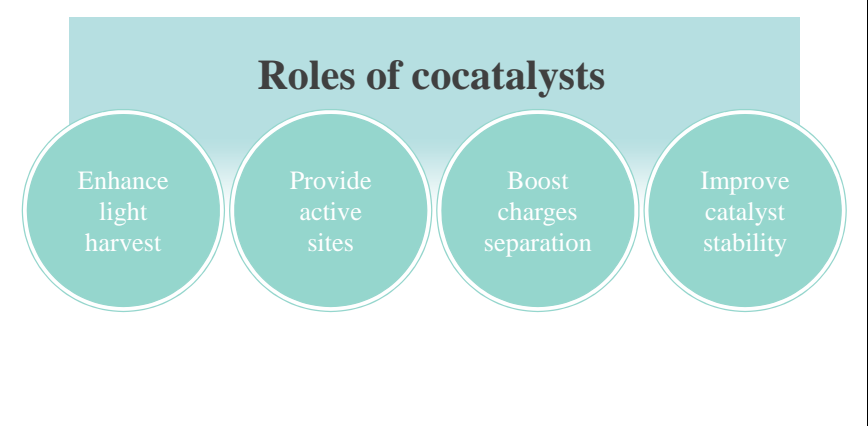

catalysts is still needed to be further studied. We believe that with the continuous development of new cocatalysts and the further development of the related theories, the efficiency of solar photocatalytic hydrogen production will continue to increase, and the photocatalytic hydrogen production system will also achieve a greater development.

\section{References}

[1] Z. Shao, T. Zeng, Y. He, D. Zhang, X. Pu, Chem. Eng. J., 2019, 359, 485-495.

[2] Z. Shao, Y. He, T. Zeng, Y. Yang, X. Pu, B. Ge, J. Dou, J. Alloys Compd., 2018, 769, 889-897.

[3] S. Liang, T. Zhang, D. Zhang, X. Pu, X. Shao, W. Li, J. Dou, J. Am. Ceram. Soc., 2018, 101, 3424-3436.

[4] T. Zhang, D. Zhao, Y. Wang, Y. Chang, D. Zhang, Y. Tang, X. Pu, X. Shao, J. Am. Ceram. Soc., 2019, 102, 3897-3907.

[5] M. Wu, J. Zhang, B.-B. He, H.-W. Wang, R. Wang, Y.-S. Gong, Appl. Catal. B: Environ., 2019, 241, 159-166.

[6] Z. Wang, C. Li, K. Domen, Chem. Soc. Rev., 2019, 48, 2109-2125.

[7] G. Xie, K. Zhang, B. Guo, Q. Liu, L. Fang, J. R. Gong, Adv. Mater., 2013, 25, 3820-3839.

[8] P. Zhang, T. Wang, X. Chang, J. Gong, Acc. Chem. Res., 2016, 49, 911-921.

[9] D. Kong, Y. Zheng, M. Kobielusz, Y. Wang, Z. Bai, W. Macyk, X. Wang, J. Tang, Mater. Today, 2018, 21, 897-924.

[10] N. Fajrina, M. Tahir, Int. J. Hydrogen Energy, 2019, 44, 540-577.

[11] Y. Wu, P. Wang, X. Zhu, Q. Zhang, Z. Wang, Y. Liu, G. Zou, Y. Dai, M.-H. Whangbo, B. Huang, Adv. Mater., 2018, 30, 1704342.

[12] A. Fujishima, K. Honda, Nature, 1972, 238, 37-38.

[13] K. Zhang, J. Ran, B. Zhu, H. Ju, J. Yu, L. Song, S.-Z. Qiao, Small, 2018, 14, 1801705.

[14] J. Fu, Q. Xu, J. Low, C. Jiang, J. Yu, Appl. Catal. B: Environ., 2019, 243, 556-565.

[15] A. Wang, D. Dai, S. Li, N. Xiao, B. Xu, Y. Gao, L. Ge, Int. J. Hydrogen Energy, 2019, 44, 8188-8196.

[16] C. Han, T. Zhang, Q. Cai, C. Ma, Z. Tong, Z. Liu, J. Am. Ceram. Soc., 2019, 102, 5484-5493.

[17] Y.-J. Yuan, P. Wang, Z. Li, Y. Wu, W. Bai, Y. Su, J. Guan, S. Wu, J. Zhong, Z.-T. Yu, Z. Zou, Appl. Catal. B: Environ., 2019, 242, 1-8.

[18] N.-C. Zheng, T. Ouyang, Y. Chen, Z. Wang, D.-Y. Chen, Z.-Q. Liu, Catal. Sci. Technol., 2019, 9, 1357-1364.

[19] J. Yu, Y. Hai, B. Cheng, J. Phys. Chem. C, 2011, 115, 4953-4958.
[20] K. E. deKrafft, C. Wang, W. Lin, Adv. Mater., 2012, 24, 2014-2018.

[21] L. Zhang, B. Tian, F. Chen, J. Zhang, Int. J. Hydrogen Energy, 2012, 37, 17060-17067.

[22] J. M. Kum, S. H. Yoo, G. Ali, S. O. Cho, Int. J. Hydrogen Energy, 2013, 38, 13541-13546.

[23] W. Chen, Y. Wang, S. Liu, L. Gao, L. Mao, Z. Fan, W. Shangguan, Z. Jiang, Appl. Surf. Sci., 2018, 445, 527-534.

[24] Q. He, H. Sun, Y. Shang, Y. Tang, P. She, S. Zeng, K. Xu, G. Lu, S. Liang, S. Yin, Z. Liu, Appl. Surf. Sci., 2018, 441, 458-465.

[25] H. Xu, D. Dai, S. Li, L. Ge, Y. Gao, Dalton. Trans., 2018, 47, 348-356.

[26] Y. Li, D. Zhang, X. Feng, Y. Liao, Q. Wen, Q. Xiang, Nanoscale Adv., 2019, 1, 1812-1818.

[27] Y. Sui, L. Wu, S. Zhong, Q. Liu, Appl. Surf. Sci., 2019, 480, 810-816.

[28] J. Zhu, J. Xiong, G. Cheng, W. Li, S. Dou, J. Colloid Interface Sci, 2019, 545, 116-127.

[29] X. Zong, H. Yan, G. Wu, G. Ma, F. Wen, L. Wang, C. Li, J. Am. Chem. Soc., 2008, 130, 7176-7177.

[30] B. Han, S. Liu, N. Zhang, Y.-J. Xu, Z.-R. Tang, Appl. Catal. B: Environ., 2017, 202, 298-304.

[31] W.-K. Jo, N. C. S. Selvam, Chem. Eng. J., 2017, 317, 913-924.

[32] S. Ma, X. Xu, J. Xie, X. Li, Chin. J. Catal., 2017, 38, 1970-1980.

[33] A. Meng, B. Zhu, B. Zhong, L. Zhang, B. Cheng, Appl. Surf. Sci., 2017, 422, 518-527.

[34] H. Cui, B. Li, Z. Li, X. Li, S. Xu, Appl. Surf. Sci., 2018, 455, 831-840.

[35] T. Hu, P. Li, J. Zhang, C. Liang, K. Dai, Appl. Surf. Sci., 2018, 442, 20-29.

[36] L. Mao, Q. Ba, S. Liu, X. Jia, H. Liu, W. Chen, X. Li, RSC Adv., 2018, 8, 31529-31537.

[37] Q. Ba, X. Jia, L. Huang, X. Li, W. Chen, L. Mao, Int. J. Hydrogen Energy, 2019, 44, 5872-5880.

[38] L. Wang, Z. Gao, Y. Li, H. She, J. Huang, B. Yu, Q. Wang, Appl. Surf. Sci., 2019, 492, 598-606.

[39] H. She, Y. Sun, S. Li, J. Huang, L. Wang, G. Zhu, Q. Wang, Appl. Catal. B: Environ., 2019, 245, 439-447.

[40] H. She, X. Ma, Z. Zhao, L. Wang, J. Huang, C. Li, Q. Wang, Appl. Surf. Sci., 2019, 489, 420-426.

[41] D. Dai, H. Xu, L. Ge, C. Han, Y. Gao, S. Li, Y. Lu, Appl. Catal. B: Environ., 2017, 217, 429-436.

[42] D. Dai, L. Wang, N. Xiao, S. Li, H. Xu, S. Liu, B. Xu, D. Lv, Y. Gao, W. Song, L. Ge, J. Liu, Appl. Catal. B: Environ., 2018, 233, 194-201.

[43] P. Zhang, T. Song, T. Wang, H. Zeng, J. Mater. Chem. A, 2017, 5, 22772-22781.

[44] B. Chai, T. Peng, J. Mao, K. Li, L. Zan, Phys. Chem. Chem. Phys., 2012, 
$14,16745-16752$.

[45] J. Hong, Y. Wang, Y. Wang, W. Zhang, R. Xu, ChemSusChem, 2013, 6, 2263-2268.

[46] J. Yu, S. Wang, B. Cheng, Z. Lin, F. Huang, Catal. Sci. Technol., 2013, 3, 1782-1789.

[47] J. Yu, S. Wang, J. Low, W. Xiao, Phys. Chem. Chem. Phys., 2013, 15, 16883-16890.

[48] X. Zhang, X. Xie, H. Wang, J. Zhang, B. Pan, Y. Xie, J. Am. Chem. Soc., 2013, 135, 18-21.

[49] J. Chen, S. Shen, P. Guo, M. Wang, P. Wu, X. Wang, L. Guo, Appl. Catal. B: Environ., 2014, 152-153, 335-341.

[50] Y. Chen, J. Li, Z. Hong, B. Shen, B. Lin, B. Gao, Phys. Chem. Chem. Phys., 2014, 16, 8106-8113.

[51] Z. Chen, P. Sun, B. Fan, Z. Zhang, X. Fang, J. Phys. Chem. C, 2014, 118, 7801-7807.

[52] F. Yang, D. Liu, Y. Li, L. Cheng, J. Ye, Appl. Catal. B: Environ., 2019, $240,64-71$.

[53] J. Zhang, M. Wu, B. He, R. Wang, H. Wang, Y. Gong, Appl. Surf. Sci., 2019, 470, 565-572.

[54] Y. Zhu, T. Wang, T. Xu, Y. Li, C. Wang, Appl. Surf. Sci., 2019, 464, $36-42$.

[55] J. Li, G. Zhan, Y. Yu, L. Zhang, Nat Commun., 2016, 7, 11480.

[56] K. Zhang, J. Ran, B. Zhu, H. Ju, J. Yu, L. Song, S. Z. Qiao, Small, 2018, 14, e1801705.

[57] L. Zhu, Q. Yue, D. Jiang, H. Chen, R. Muhammad Irfan, P. Du, Chin. J. Catal., 2018, 39, 1753-1761.

[58] Q. Han, F. Zhao, C. Hu, L. Lv, Z. Zhang, N. Chen, L. Qu, Nano Res., 2015, 8, 1718-1728.

[59] N. Tian, Y. Zhang, X. Li, K. Xiao, X. Du, F. Dong, G. I. N. Waterhouse, T. Zhang, H. Huang, Nano Energy, 2017, 38, 72-81.

[60] X. Yang, F. Qian, G. Zou, M. Li, J. Lu, Y. Li, M. Bao, Appl. Catal. B: Environ., 2016, 193, 22-35.

[61] C. Ji, S.-N. Yin, S. Sun, S. Yang, Appl. Surf. Sci., 2018, 434, 1224-1231.

[62] G. Liu, G. Zhao, W. Zhou, Y. Liu, H. Pang, H. Zhang, D. Hao, X. Meng, P. Li, T. Kako, J. Ye, Adv. Funct. Mater., 2016, 26, 6822-6829.

[63] D. K. L. Chan, J. C. Yu, Catal. Today, 2018, 310, 26-31.

[64] L. Kong, X. Mu, X. Fan, R. Li, Y. Zhang, P. Song, F. Ma, M. Sun, Appl. Mater. Today, 2018, 13, 329-338.

[65] J. Cao, W. Nie, L. Huang, Y. Ding, K. Lv, H. Tang, Appl. Catal. B: Environ., 2019, 241, 18-27.

[66] H. Yu, R. Shi, Y. Zhao, T. Bian, Y. Zhao, C. Zhou, G. I. N. Waterhouse, L. Z. Wu, C. H. Tung, T. Zhang, Adv. Mater., 2017, 29, 1605148.

[67] J.-W. Zhang, S. Gong, N. Mahmood, L. Pan, X. Zhang, J.-J. Zou, Appl. Catal. B: Environ., 2018, 221, 9-16.

[68] S. Guo, Y. Tang, Y. Xie, C. Tian, Q. Feng, W. Zhou, B. Jiang, Appl. Catal. B: Environ., 2017, 218, 664-671.

[69] X. Yang, J. Xu, T. Wong, Q. Yang, C. S. Lee, Phys. Chem. Chem. Phys., 2013, 15, 12688-12693.

[70] L. Huang, X. Wang, J. Yang, G. Liu, J. Han, C. Li, J. Phys. Chem. C, 2013, 117, 11584-11591.

[71] J. Zhang, S. Z. Qiao, L. Qi, J. Yu, Phys. Chem. Chem. Phys., 2013, 15, 12088-12094.

[72] S. G. Babu, R. Vinoth, D. P. Kumar, M. V. Shankar, H. L. Chou, K. Vinodgopal, B. Neppolian, Nanoscale, 2015, 7, 7849-7857.

[73] A. Samal, S. Swain, U. Manju, D. P. Das, ACS Sustain. Chem. Eng., 2019, 7, 10052-10063.

[74] X. She, H. Xu, Y. Yu, L. Li, X. Zhu, Z. Mo, Y. Song, J. Wu, S. Yuan, H. Li, Small, 2019, 15, 1804613.

[75] N. Fajrina, M. Tahir, Appl. Surf. Sci., 2019, 471, 1053-1064.

[76] R. Zhu, F. Tian, R. Yang, J. He, J. Zhong, B. Chen, Renew. Energy, 2019, 139, 22-27.
[77] S. Chen, F. Li, T. Li, W. Cao, J. Colloid Interface Sci., 2019, 547, 50-59.

[78] Y. Jiao, Q. Huang, J. Wang, Z. He, Z. Li, Appl. Catal. B: Environ., 2019, 247, 124-132.

[79] J. Yang, D. Wang, H. Han, C. Li, Acc. Chem. Res., 2013, 46, 1900-1909.

[80] R. Li, C. Li, Adv. Catal., 2017, 60, 1-57.

[81] A. Meng, L. Zhang, B. Cheng, J. Yu, Adv. Mater., 2019, 31, 1807660

[82] S. Zhao, Y. Zhang, Y. Zhou, J. Fang, Y. Wang, C. Zhang, W. Chen, J. Mater. Sci., 2018, 53, 6008-6020.

[83] J. D. Xiao, L. Han, J. Luo, S. H. Yu, H. L. Jiang, Angew. Chem. Int. Ed. Engl., 2018, 57, 1103-1107.

[84] X. Wang, H. Wang, H. Zhang, W. Yu, X. Wang, Y. Zhao, X. Zong, C. $\mathrm{Li}$, ACS Energy Lett., 2018, 3, 1159-1164.

[85] K. He, J. Xie, Z. Yang, R. Shen, Y. Fang, S. Ma, X. Chen, X. Li, Catal. Sci. Technol., 2017, 7, 1193-1202.

[86] H. Chen, D. Jiang, Z. Sun, R. M. Irfan, L. Zhang, P. Du, Catal. Sci. Technol., 2017, 7, 1515-1522.

[87] J. Ran, J. Zhang, J. Yu, M. Jaroniec, S. Z. Qiao, Chem. Soc. Rev., 2014, 43, 7787-7812.

[88] S. J. A. Moniz, S. A. Shevlin, D. J. Martin, Z.-X. Guo, J. Tang, Energy Environ. Sci., 2015, 8, 731-759.

[89] S.-S. Yi, X.-B. Zhang, B.-R. Wulan, J.-M. Yan, Q. Jiang, Energy Environ. Sci., 2018, 11, 3128-3156.

[90] X. Li, W. Bi, L. Zhang, S. Tao, W. Chu, Q. Zhang, Y. Luo, C. Wu, Y. Xie, Adv. Mater., 2016, 28, 2427-2431.

[91] Y. Cao, D. Wang, Y. Lin, W. Liu, L. Cao, X. Liu, W. Zhang, X. Mou, S. Fang, X. Shen, T. Yao, ACS Appl. Energy Mater., 2018, 1, 6082-6088.

[92] M. Wu, J.-M. Yan, X.-w. Zhang, M. Zhao, Appl. Surf. Sci., 2015, 354, 196-200.

[93] M. Z. Rahman, J. Ran, Y. Tang, M. Jaroniec, S. Z. Qiao, J. Mater. Chem. A, 2016, 4, 2445-2452.

[94] Y. Zhong, Z. Wang, J. Feng, S. Yan, H. Zhang, Z. Li, Z. Zou, Appl. Surf. Sci., 2014, 295, 253-259.

[95] M. Ou, S. Wan, Q. Zhong, S. Zhang, Y. Wang, Int. J. Hydrogen Energy, 2017, 42, 27043-27054.

[96] M. Liu, P. Xia, L. Zhang, B. Cheng, J. Yu, ACS Sustain. Chem. Eng., 2018, 6, 10472-10480.

[97] Z. H. N. Al-Azri, W.-T. Chen, A. Chan, V. Jovic, T. Ina, H. Idriss, G. I. N. Waterhouse, J. Catal., 2015, 329, 355-367.

[98] Z. Mo, H. Xu, X. She, Y. Song, P. Yan, J. Yi, X. Zhu, Y. Lei, S. Yuan, H. Li, Appl. Surf. Sci., 2019, 467-468, 151-157.

[99] A. Caravaca, W. Jones, C. Hardacre, M. Bowker, Proc. Math. Phys. Eng. Sci., 2016, 472, 20160054.

[100] T. Chen, W. Quan, L. Yu, Y. Hong, C. Song, M. Fan, L. Xiao, W. Gu, W. Shi, J. Alloys Compd., 2016, 686, 628-634.

[101] P. V. Korake, S. N. Achary, N. M. Gupta, Int. J. Hydrogen Energy, 2015, 40, 8695-8705.

[102] A. J. Cheah, W. S. Chiu, P. S. Khiew, H. Nakajima, T. Saisopa, P. Songsiriritthigul, S. Radiman, M. A. A. Hamid, Catal. Sci. Technol., 2015, 5, 4133-4143.

[103] J. Qin, J. Huo, P. Zhang, J. Zeng, T. Wang, H. Zeng, Nanoscale, 2016, 8, 2249-2259.

[104] Y. Guo, H. Jia, J. Yang, H. Yin, Z. Yang, J. Wang, B. Yang, Phys. Chem. Chem. Phys., 2018, 20, 22296-22307.

[105] Y. Si, S. Cao, Z. Wu, Y. Ji, Y. Mi, X. Wu, X. Liu, L. Piao, Appl. Catal. B: Environ., 2018, 220, 471-476.

[106] W.-T. Chen, A. Chan, D. Sun-Waterhouse, J. Llorca, H. Idriss, G. I. N. Waterhouse, J. Catal., 2018, 367, 27-42.

[107] B. Liu, Y. Wang, S. Shang, Y. Ni, N. Zhang, M. Cao, C. Hu, Colloids 
Surf. A: Physicochem. Eng. Asp., 2018, 553, 203-209.

[108] X.-B. Qian, W. Peng, J.-H. Huang, Mater. Res. Bull., 2018, 102, 362-368.

[109] X. Meng, L. Liu, S. Ouyang, H. Xu, D. Wang, N. Zhao, J. Ye, Adv. Mater., 2016, 28, 6781-6803.

[110] Z. Zhang, S.-W. Cao, Y. Liao, C. Xue, Appl. Catal. B: Environ., 2015, $162,204-209$.

[111] S. Li, L. Hou, L. Zhang, L. Chen, Y. Lin, D. wang, T. Xie, J. Mater. Chem. A, 2015, 3, 17820-17826.

[112] Z. Jiao, Y. Zhang, S. Ouyang, H. Yu, G. Lu, J. Ye, Y. Bi, ACS Appl. Mater. Interfaces, 2014, 6, 19488-19493.

[113] S. Zhu, X. Xie, S.-C. Chen, S. Tong, G. Lu, D. Y. H. Pui, J. Sun, Appl. Surf. Sci., 2017, 408, 117-124.

[114] L. Vila, P. Vincent, L. Dauginet-De Pra, G. Pirio, E. Minoux, L. Gangloff, S. Demoustier-Champagne, N. Sarazin, E. Ferain, R. Legras, L. Piraux, P. Legagneux, Nano Lett., 2004, 4, 521-524.

[115] W. Ye, R. Long, H. Huang, Y. Xiong, J. Mater. Chem. C, 2017, 5, 1008-1021.

[116] P. Zhang, T. Song, T. Wang, H. Zeng, Appl. Catal. B: Environ., 2018, 225, 172-179.

[117] P. Zhang, T. Song, T. Wang, H. Zeng, Appl. Catal. B: Environ., 2017, 206, 328-335.

[118] B. Yue, Q. Li, H. Iwai, T. Kako, J. Ye, Sci. Technol. Adv. Mater., 2011, $12,034401$.

[119] W.-T. Chen, A. Chan, D. Sun-Waterhouse, T. Moriga, H. Idriss, G. I. N. Waterhouse, J. Catal., 2015, 326, 43-53.

[120] L. Bi, D. Xu, L. Zhang, Y. Lin, D. Wang, T. Xie, Phys. Chem. Chem. Phys., 2015, 17, 29899-29905.

[121] L. Kong, Y. Dong, P. Jiang, G. Wang, H. Zhang, N. Zhao, J. Mater. Chem. A, 2016, 4, 9998-10007.

[122] M. H. Vu, C. C. Nguyen, M. Sakar, T. O. Do, Phys. Chem. Chem. Phys., 2017, 19, 29429-29437.

[123] S. Chen, X. Chen, Q. Jiang, J. Yuan, C. Lin, W. Shangguan, Appl. Surf. Sci., 2014, 316, 590-594.

[124] N. Zhao, L. Kong, Y. Dong, G. Wang, X. Wu, P. Jiang, ACS Appl. Mater. Interfaces, 2018, 10, 9522-9531.

[125] B. Yue, Q. Li, H. Iwai, T. Kako, J. Ye, Sci. Technol. Adv. Mater., 2011, $12,034401$.

[126] H.-Y. Lin, H.-C. Yang, W.-L. Wang, Catal. Today, 2011, 174, 106-113.

[127] N. Xiao, S. Li, S. Liu, B. Xu, Y. Li, Y. Gao, L. Ge, G. Lu, Chin. J. Catal,, 2019, 40, 352-361.

[128] C. Han, Y. Lu, J. Zhang, L. Ge, Y. Li, C. Chen, Y. Xin, L. Wu, S. Fang, J. Mater. Chem. A, 2015, 3, 23274-23282.

[129] C. Han, L. Wu, L. Ge, Y. Li, Z. Zhao, Carbon, 2015, 92, 31-40.

[130] C. Han, Y. Gao, S. Liu, L. Ge, N. Xiao, D. Dai, B. Xu, C. Chen, Int. J. Hydrogen Energy, 2017, 42, 22765-22775.

[131] Y. Zhu, A. Marianov, H. Xu, C. Lang, Y. Jiang, ACS Appl. Mater. Interfaces, 2018, 10, 9468-9477.

[132] B. Wang, S. He, L. Zhang, X. Huang, F. Gao, W. Feng, P. Liu, Appl. Catal. B: Environ., 2019, 243, 229-235.

[133] X. Han, D. Xu, L. An, C. Hou, Y. Li, Q. Zhang, H. Wang, Appl. Catal. B: Environ., 2019, 243, 136-144.

[134] X. Yue, S. Yi, R. Wang, Z. Zhang, S. Qiu, Appl. Catal. B: Environ., 2018, 224, 17-26.

[135] J. Ding, X. Li, L. Chen, X. Zhang, X. Tian, Appl. Catal. B: Environ., 2018, 224, 322-329.

[136] A. L. Luna, D. Dragoe, K. Wang, P. Beaunier, E. Kowalska, B. Ohtani, D. Bahena Uribe, M. A. Valenzuela, H. Remita, C. Colbeau-Justin, J. Phys.l Chem. C, 2017, 121, 14302-14311.

[137] A. L. Luna, E. Novoseltceva, E. Louarn, P. Beaunier, E. Kowalska, B. Ohtani, M. A. Valenzuela, H. Remita, C. Colbeau-Justin, Appl.
Catal. B: Environ., 2016, 191, 18-28.

[138] W. Peng, S.-S. Zhang, Y.-B. Shao, J.-H. Huang, Int. J. Hydrogen Energy, 2018, 43, 22215-22225.

[139] K. K. M, B. K, N. G, S. B, V. A, Appl. Catal. B: Environ., 2016, 199, 282-291.

[140] A. A. Melvin, K. Illath, T. Das, T. Raja, S. Bhattacharyya, C. S. Gopinath, Nanoscale, 2015, 7, 13477-13488.

[141] Z. Lin, J. Li, L. Li, L. Yu, W. Li, G. Yang, J. Mater. Chem. A, 2017, 5, 773-781.

[142] M. H. Hansen, L. A. Stern, L. Feng, J. Rossmeisl, X. Hu, Phys. Chem. Chem. Phys., 2015, 17, 10823-10829.

[143] Y. Pei, Y. Cheng, J. Chen, W. Smith, P. Dong, P. M. Ajayan, M. Ye, J. Shen, J. Mater. Chem. A, 2018, 6, 23220-23243.

[144] S. Li, L. Wang, S. Liu, B. Xu, N. Xiao, Y. Gao, W. Song, L. Ge, J. Liu, ACS Sustain. Chem. Eng., 2018, 6, 9940-9950.

[145] D. Zeng, W.-J. Ong, H. Zheng, M. Wu, Y. Chen, D.-L. Peng, M.-Y. Han, J. Mater. Chem. A, 2017, 5, 16171-16178.

[146] R. Shen, J. Xie, X. Lu, X. Chen, X. Li, ACS Sustain. Chem. Eng., 2018, 6, 4026-4036.

[147] H. Cheng, X. J. Lv, S. Cao, Z. Y. Zhao, Y. Chen, W. F. Fu, Sci. Rep., 2016, 6, 19846.

[148] Z. Qin, Y. Chen, Z. Huang, J. Su, L. Guo, J. Mater. Chem. A, 2017, 5, 19025-19035.

[149] Z. Sun, M. Zhu, M. Fujitsuka, A. Wang, C. Shi, T. Majima, ACS Appl. Mater. Interfaces, 2017, 9, 30583-30590.

[150] H. Zhao, J. Wang, Y. Dong, P. Jiang, ACS Sustain. Chem. Eng., 2017, 5, 8053-8060.

[151] J. Zhang, W. Yao, C. Huang, P. Shi, Q. Xu, J. Mater. Chem. A, 2017, 5, 12513-12519.

[152] W. Bi, L. Zhang, Z. Sun, X. Li, T. Jin, X. Wu, Q. Zhang, Y. Luo, C. Wu, Y. Xie, ACS Catal., 2016, 6, 4253-4257.

[153] H. Zhao, P. Jiang, W. Cai, Chem. Asian J, 2017, 12, 361-365.

[154] D. Zeng, W. Xu, W.-J. Ong, J. Xu, H. Ren, Y. Chen, H. Zheng, D.-L. Peng, Appl. Catal. B: Environ., 2018, 221, 47-55.

[155] H. Zhao, S. Sun, P. Jiang, Z. J. Xu, Chem. Eng. J., 2017, 315, 296-303.

[156] C. Liu, K. Wu, G. Meng, J. Wu, B. Peng, J. Hou, Z. Liu, X. Guo, Mol. Catal., 2017, 437, 80-88.

[157] Y. Chen, Z. Qin, Catal. Sci. Technol., 2016, 6, 8212-8221.

[158] Z. Jiang, C. Zhu, W. Wan, K. Qian, J. Xie, J. Mater. Chem. A, 2016, 4, 1806-1818.

[159] C. Yang, J. Qin, Z. Xue, M. Ma, X. Zhang, R. Liu, Nano Energy, 2017, 41, 1-9.

[160] Y. Tan, Z. Shu, J. Zhou, T. Li, W. Wang, Z. Zhao, Appl. Catal. B: Environ., 2018, 230, 260-268.

[161] J. Pan, Z. Dong, B. Wang, Z. Jiang, C. Zhao, J. Wang, C. Song, Y. Zheng, C. Li, Appl. Catal. B: Environ., 2019, 242, 92-99.

[162] J. Yu, Y. Hai, M. Jaroniec, J. Colloid Interface Sci,, 2011, 357, 223-228.

[163] J. Qian, Z. Chen, F. Chen, Y. Wang, Z. Wu, W. Zhang, Z. Wu, P. Li, Inorg. Chem., 2018, 57, 14532-14541.

[164] D. Praveen Kumar, M. V. Shankar, M. M. Kumari, G. Sadanandam, B. Srinivas, V. Durgakumari, Chem. Commun., 2013, 49, 9443-9445.

[165] C. Chen, Y. Zhan, D. Li, Y. Zhang, X. Lin, L. Jiang, Q. Zheng, Energy Technol., 2018, 6, 1096-1103.

[166] L. Li, L. Xu, W. Shi, J. Guan, Int. J. Hydrogen Energy, 2013, 38, 816-822.

[167] Y. Li, B. Wang, S. Liu, X. Duan, Z. Hu, Appl. Surf. Sci., 2015, 324, 736-744.

[168] L. Li, B. Cheng, Y. Wang, J. Yu, J. Colloid Interf. Sci., 2015, 449, 115-121. 
[169] H. Xu, S. Li, L. Ge, C. Han, Y. Gao, D. Dai, Int. J. Hydrogen Energy, 2017, 42, 22877-22886.

[170] M. Wu, J.-M. Yan, X.-W. Zhang, M. Zhao, Q. Jiang, J. Mater. Chem. A, 2015, 3, 15710-15714.

[171] J. Ran, J. Yu, M. Jaroniec, Green Chem., 2011, 13, 2708-2713.

[172] Q. Wang, D. O'Hare, Chem. Rev., 2012, 112, 4124-4155.

[173] S. Nayak, L. Mohapatra, K. Parida, J. Mater. Chem. A, 2015, 3, 18622-18635.

[174] Y. Qiu, B. Lin, F. Jia, Y. Chen, B. Gao, P. Liu, Mater. Res. Bull., 2015, 72, 235-240.

[175] X. L. Guo, X. Y. Liu, X. D. Hao, S. J. Zhu, F. Dong, Z. Q. Wen, Y. X. Zhang, Electrochim. Acta, 2016, 194, 179-186.

[176] C. Chen, K. Ruengkajorn, J.-C. Buffet, D. O'Hare, RSC Adv., 2018, 8, 34650-34655.

[177] G. Zhang, B. Lin, Y. Qiu, L. He, Y. Chen, B. Gao, Int. J. Hydrogen Energy, 2015, 40, 4758-4765.

[178] J. M. Lee, J. H. Yang, N. H. Kwon, Y. K. Jo, J. H. Choy, S. J. Hwang, Dalton. Trans., 2018, 47, 2949-2955.

[179] N. Lakshmana Reddy, S. Emin, M. Valant, M. V. Shankar, Int. J. Hydrogen Energy, 2017, 42, 6627-6636.

[180] L. Mao, Q. Ba, X. Jia, S. Liu, H. Liu, J. Zhang, X. Li, W. Chen, RSC Adv., 2019, 9, 1260-1269.

[181] X. Zhou, Z. Luo, P. Tao, B. Jin, Z. Wu, Y. Huang, Mater. Chem. Phys., 2014, 143, 1462-1468.

[182] J. Yu, J. Ran, Energy Environ. Sci., 2011, 4, 1364-1371.

[183] S. Li, L. Wang, Y. Li, L. Zhang, A. Wang, N. Xiao, Y. Gao, N. Li, W. Song, L. Ge, J. Liu, Appl. Catal. B: Environ., 2019, 254, 145-155.

[184] G. Chen, N. Ding, F. Li, Y. Fan, Y. Luo, D. Li, Q. Meng, Appl. Catal. B: Environ., 2014, 160-161, 614-620.

[185] Y. Liu, X. Xu, J. Zhang, H. Zhang, W. Tian, X. Li, M. O. Tade, H. Sun, S. Wang, Appl. Catal. B: Environ., 2018, 239, 334-344.

[186] B. Chai, C. Liu, C. Wang, J. Yan, Z. Ren, Chin. J. Catal., 2017, 38, 2067-2075.

[187] Y.-J. Yuan, D. Chen, J. Zhong, L.-X. Yang, J. Wang, M.-J. Liu, W.-G. Tu, Z.-T. Yu, Z.-G. Zou, J. Mater. Chem. A, 2017, 5, 15771-15779.

[188] M.-L. Xu, X. Tang, Y. Wang, F.-T. Liu, Y. Zhang, K. Li, New J. Chem., 2019, 43, 5335-5340.

[189] K. He, J. Xie, M. Li, X. Li, Appl. Surf. Sci., 2018, 430, 208-217.

[190] J. Zhang, W. Li, Y. Li, L. Zhong, C. Xu, Appl. Catal. B: Environ., 2017, 217, 30-36.

[191] J. Zhang, J. Yu, Y. Zhang, Q. Li, J. R. Gong, Nano Lett., 2011, 11, 4774-4779.

[192] T. Chen, C. Song, M. Fan, Y. Hong, B. Hu, L. Yu, W. Shi, Int. J. Hydrogen Energy, 2017, 42, 12210-12219.

[193] Y. Chen, Z. Qin, X. Wang, X. Guo, L. Guo, RSC Adv., 2015, 5, 18159-18166.

[194] L. Zhang, T. Jiang, S. Li, Y. Lu, L. Wang, X. Zhang, D. Wang, T. Xie, Dalton. Trans., 2013, 42, 12998-13003.

[195] L. J. Zhang, T. F. Xie, D. J. Wang, S. Li, L. L. Wang, L. P. Chen, Y. C. Lu, Int. J. Hydrogen Energy, 2013, 38, 11811-11817.

[196] Q. Wang, N. An, Y. Bai, H. Hang, J. Li, X. Lu, Y. Liu, F. Wang, Z. Li, Z. Lei, Int. J. Hydrogen Energy, 2013, 38, 10739-10745.

[197] F. Zhang, H.-Q. Zhuang, J. Song, Y.-L. Men, Y.-X. Pan, S.-H. Yu, Appl. Catal. B: Environ., 2018, 226, 103-110.

[198] J. Jia, Q. Zhang, Z. Li, X. Hu, E. Liu, J. Fan, J. Mater. Chem. A, 2019, 7, 3828-3841.

[199] D. Jiang, L. Chen, J. Xie, M. Chen, Dalton. Trans., 2014, 43, 4878-4885.

[200] C. García-Mendoza, S. Oros-Ruiz, A. Hernández-Gordillo, R. López, G. Jácome-Acatitla, H. A. Calderón, R. Gómez, J. Chem. Technol. Biotechnol., 2016, 91, 2198-2204.

[201] M. S. Akple, J. Low, S. Wageh, A. A. Al-Ghamdi, J. Yu, J. Zhang, Appl.
Surf. Sci., 2015, 358, 196-203.

[202] X. Ma, J. Li, C. An, J. Feng, Y. Chi, J. Liu, J. Zhang, Y. Sun, Nano Res., 2016, 9, 2284-2293.

[203] Y. Chao, J. Zheng, J. Chen, Z. Wang, S. Jia, H. Zhang, Z. Zhu, Catal. Sci. Technol., 2017, 7, 2798-2804.

[204] W. Zhang, Y. Wang, Z. Wang, Z. Zhong, R. Xu, Chem. Commun., 2010, 46, 7631-7633.

[205] S. Meng, Y. Cui, H. Wang, X. Zheng, X. Fu, S. Chen, Dalton. Trans., 2018, 47, 12671-12683.

[206] J. Wang, B. Li, J. Chen, N. Li, J. Zheng, J. Zhao, Z. Zhu, Appl. Surf. Sci., 2012, 259, 118-123.

[207] W. Liu, X. Wang, H. Yu, J. Yu, ACS Sustain. Chem. Eng., 2018, 6, 12436-12445.

[208] Y. Chen, S. Zhao, X. Wang, Q. Peng, R. Lin, Y. Wang, R. Shen, X. Cao, L. Zhang, G. Zhou, J. Li, A. Xia, Y. Li, J. Am. Chem. Soc., 2016, 138, 4286-4289.

[209] B. Lin, H. Li, H. An, W. Hao, J. Wei, Y. Dai, C. Ma, G. Yang, Appl. Catal. B: Environ., 2018, 220, 542-552.

[210] C. Liu, L. Wang, Y. Tang, S. Luo, Y. Liu, S. Zhang, Y. Zeng, Y. Xu, Appl. Catal. B: Environ., 2015, 164, 1-9.

[211] X. Xia, N. Deng, G. Cui, J. Xie, X. Shi, Y. Zhao, Q. Wang, W. Wang, B. Tang, Chem. Commun., 2015, 51, 10899-10902.

[212] X. Wang, J. Cheng, H. Yu, J. Yu, Dalton. Trans., 2017, 46, 6417-6424.

[213] Y. Wang, X. Liu, J. Liu, B. Han, X. Hu, F. Yang, Z. Xu, Y. Li, S. Jia, Z. Li, Y. Zhao, Angew. Chem. Int. Ed., 2018, 57, 5765-5771.

[214] Q. Xiang, J. Yu, M. Jaroniec, J. Phys. Chem. C, 2011, 115, 7355-7363.

[215] Y.-H. Zhang, X.-L. Cai, D.-Y. Guo, H.-J. Zhang, N. Zhou, S.-M. Fang, J.-L. Chen, H.-L. Zhang, J. Mater. Sci.: Mater. Electron., 2019, 30, 7182-7193.

[216] N. Ramesh Reddy, M. Mamatha Kumari, K. K. Cheralathan, M. V. Shankar, Int. J. Hydrogen Energy, 2018, 43, 4036-4043.

[217] Z. Yao, L. Wang, Y. Zhang, Z. Yu, Z. Jiang, Int. J. Hydrogen Energy, 2014, 39, 15380-15386.

[218] T. Zhu, H. B. Wu, Y. Wang, R. Xu, X. W. D. Lou, Adv. Energy Mater., 2012, 2, 1497-1502.

[219] J. Yu, B. Yang, B. Cheng, Nanoscale, 2012, 4, 2670-2677.

[220] L. Song, X. Kang, S. Zhang, Int. J. Energy Res., 2018, 42, 1649-1656.

[221] L. Wang, Z. Yao, F. Jia, B. Chen, Z. Jiang, Dalton. Trans., 2013, 42, 9976-9981.

[222] A. K. Geim, K. S. Novoselov, Nat. Mater., 2007, 6, 183.

[223] A. K. Geim, Science, 2009, 324, 1530.

[224] M. J. Allen, V. C. Tung, R. B. Kaner, Chem. Rev., 2010, 110, 132-145.

[225] J. Dong, Y. Shi, C. Huang, Q. Wu, T. Zeng, W. Yao, Appl. Catal. B: Environ., 2019, 243, 27-35.

[226] K. Maeda, A. Xiong, T. Yoshinaga, T. Ikeda, N. Sakamoto, T. Hisatomi, M. Takashima, D. Lu, M. Kanehara, T. Setoyama, T. Teranishi, K. Domen, Angew. Chem. Int. Ed., 2010, 49, 4096-4099.

[227] A. Ibhadon, P. Fitzpatrick, Catalysts, 2013, 3, 189-218.

[228] J. Yang, H. Yan, X. Zong, F. Wen, M. Liu, C. Li, Phil. Trans. R. Soc., 2013, 371, 20110430.

[229] H. Yu, X. Huang, P. Wang, J. Yu, J. Phys. Chem. C, 2016, 120, 3722-3730.

[230] F. Wang, Y. Su, S. Min, Y. Li, Y. Lei, J. Hou, J. Solid State Chem., 2018, 260, 23-30.

[231] Z. Wang, W. Wu, Q. Xu, G. Li, S. Liu, X. Jia, Y. Qin, Z. L. Wang, Nano Energy, 2017, 38, 518-525.

[232] A. Li, T. Wang, X. Chang, W. Cai, P. Zhang, J. Zhang, J. Gong, Chem. 
Sci., 2016, 7, 890-895.

[233] H. Yan, J. Yang, G. Ma, G. Wu, X. Zong, Z. Lei, J. Shi, C. Li, J. Catal., 2009, 266, 165-168.

[234] Q. Sun, N. Wang, J. Yu, J. C. Yu, Adv. Mater., 2018, 30, 1804368.

[235] J. Zhang, Z. Yu, Z. Gao, H. Ge, S. Zhao, C. Chen, S. Chen, X. Tong, M. Wang, Z. Zheng, Y. Qin, Angew. Chem. Int. Ed., 2017, 56, 816-820.

[236] J. Wang, J. Luo, D. Liu, S. Chen, T. Peng, Appl. Catal. B: Environ., 2019, 241, 130-140.

[237] C.-J. Chang, Y.-G. Lin, H.-T. Weng, Y.-H. Wei, Appl. Surf. Sci., 2018, 451, 198-206.

[238] X. Ding, Y. Li, J. Zhao, Y. Zhu, Y. Li, W. Deng, C. Wang, APL Mater., 2015, 3, 104410.

[239] J. Lang, M. Liu, Y. Su, L. Yan, X. Wang, Appl. Surf. Sci., 2015, 358, 252-260.

[240] P. Wang, Z. Guan, Q. Li, J. Yang, J. Mater. Sci., 2017, 53, 774-786.

[241] G. Zhang, G. Li, X. Wang, ChemCatChem, 2015, 7, 2864-2870.

[242] X. Chen, S. Chen, C. Lin, Z. Jiang, W. Shangguan, Int. J. Hydrogen Energy, 2015, 40, 998-1004.

[243] Q. Wang, G. Yun, Y. Bai, N. An, Y. Chen, R. Wang, Z. Lei, W. Shangguan, Int. J. Hydrogen Energy, 2014, 39, 13421-13428.

[244] X. Yue, S. Yi, R. Wang, Z. Zhang, S. Qiu, Sci. Rep., 2016, 6, 22268.

[245] H. Zhao, M. Wu, J. Liu, Z. Deng, Y. Li, B.-L. Su, Appl. Catal. B: Environ., 2016, 184, 182-190.

[246] R. Tong, C. Liu, Z. Xu, Q. Kuang, Z. Xie, L. Zheng, ACS Appl. Mater. Interfaces, 2016, 8, 21326-21333.

[247] E. Hong, D. Kim, J. H. Kim, J. Ind. Eng. Chem., 2014, 20, 3869-3874.

[248] J. Wang, Z. Wang, Z. Zhu, Appl. Catal. B: Environ., 2017, 204, 577-583.

[249] D. P. Sahoo, S. Patnaik, D. Rath, B. Nanda, K. Parida, RSC Adv., 2016, 6, 112602-112613.

[250] W. J. Foo, C. Zhang, G. W. Ho, Nanoscale, 2013, 5, 759-764.

[251] D. Lu, Q. Wang, K. K. Kondamareddy, A. Wang, H. Hao, Q. Wu, J. Alloys Compd., 2018, 761, 31-40.

[252] A. J. Bard, J. Photochem., 1979, 10, 59-75.

[253] Y. L. Wang, Y. H. Li, X. L. Wang, Y. Hou, A. P. Chen, H. G. Yang, Appl. Catal. B: Environ., 2017, 206, 216-220.

[254] R. Niishiro, S. Tanaka, A. Kudo, Appl. Catal. B: Environ., 2014, 150-151, 187-196.

[255] Y. Sasaki, A. Iwase, H. Kato, A. Kudo, J. Catal., 2008, 259, 133-137.

[256] G. Ma, S. Chen, Y. Kuang, S. Akiyama, T. Hisatomi, M. Nakabayashi, N. Shibata, M. Katayama, T. Minegishi, K. Domen, J. Phys. Chem. Lett., 2016, 7, 3892-3896.

[257] K. Sayama, R. Abe, H. Arakawa, H. Sugihara, Catal. Commun., 2006, 7, 96-99.

[258] A. Iwase, A. Kudo, Chem. Commun., 2017, 53, 6156-6159.

[259] P. Yang, J. Zhao, J. Wang, B. Cao, L. Li, Z. Zhu, J. Mater. Chem. A, 2015, 3, 8256-8259.

[260] H. Zhao, X. Ding, B. Zhang, Y. Li, C. Wang, Sci. Bull., 2017, 62, 602-609.

[261] H. Gao, P. Zhang, J. Zhao, Y. Zhang, J. Hu, G. Shao, Appl. Catal. B: Environ., 2017, 210, 297-305.

[262] W. Xue, X. Hu, E. Liu, J. Fan, Appl. Surf. Sci., 2018, 447, 783-794.

[263] B.-J. Ng, L. K. Putri, L.-L. Tan, P. Pasbakhsh, S.-P. Chai, Chem. Eng. J., 2017, 316, 41-49.

[264] Q. Xu, L. Zhang, J. Yu, S. Wageh, A. A. Al-Ghamdi, M. Jaroniec, Mater. Today, 2018, 21, 1042-1063.

[265] X. Ma, Q. Jiang, W. Guo, M. Zheng, W. Xu, F. Ma, B. Hou, RSC Adv., 2016, 6, 28263-28269.

[266] J. W. Shi, Y. Zou, D. Ma, Z. Fan, L. Cheng, D. Sun, Z. Wang, C. Niu, L.
Wang, Nanoscale, 2018, 10, 9292-9303.

[267] S. Liang, B. Han, X. Liu, W. Chen, M. Peng, G. Guan, H. Deng, Z. Lin, J. Alloys Compd., 2018, 754, 105-113.

[268] L. Qian, Y. Hou, Z. Yu, M. Li, F. Li, L. Sun, W. Luo, G. Pan, Mol. Catal., 2018, 458, 43-51.

[269] S.-R. Kim, W.-K. Jo, Int. J. Hydrogen Energy, 2019, 44, 801-808.

[270] Z. Ai, Y. Shao, B. Chang, B. Huang, Y. Wu, X. Hao, Appl. Catal. B: Environ., 2019, 242, 202-208.

[271] X. L. Yin, J. Liu, W. J. Jiang, X. Zhang, J. S. Hu, L. J. Wan, Chem. Commun., 2015, 51, 13842-13845.

[272] D. Zheng, C. Pang, X. Wang, Chem. Commun., 2015, 51, 17467-17470.

[273] J. Zhao, P. Zhang, J. Fan, J. Hu, G. Shao, Appl. Surf. Sci., 2018, 430, 466-474.

[274] F. Xu, L. Zhang, B. Cheng, J. Yu, ACS Sustain. Chem. Eng., 2018, 6, 12291-12298.

[275] M. Arif, Z. Min, L. Yuting, H. Yin, X. Liu, J. Ind. Eng. Chem., 2019, 69, 345-357.

[276] D. Ma, J. W. Shi, Y. Zou, Z. Fan, X. Ji, C. Niu, ACS Appl. Mater. Interfaces, 2017, 9, 25377-25386.

[277] L. J. Zhang, S. Li, B. K. Liu, D. J. Wang, T. F. Xie, ACS Catal., 2014, 4, 3724-3729.

[278] F. Mei, J. Zhang, K. Dai, G. Zhu, C. Liang, Dalton. Trans., 2019, 48, 1067-1074.

[279] Y. Che, B. Lu, Q. Qi, H. Chang, J. Zhai, K. Wang, Z. Liu, Sci. Rep., 2018, 8, 16504.

[280] F. Xu, W. Xiao, B. Cheng, J. Yu, Int. J. Hydrogen Energy, 2014, 39, 15394-15402.

[281] X. Xing, M. Zhang, L. Hou, L. Xiao, Q. Li, J. Yang, Int. J. Hydrogen Energy, 2017, 42, 28434-28444.

[282] X. Han, D. Xu, L. An, C. Hou, Y. Li, Q. Zhang, H. Wang, Int. J. Hydrogen Energy, 2018, 43, 4845-4855.

[283] Z.-F. Huang, J. Song, X. Wang, L. Pan, K. Li, X. Zhang, L. Wang, J.-J. Zou, Nano Energy, 2017, 40, 308-316.

[284] K. He, J. Xie, X. Luo, J. Wen, S. Ma, X. Li, Y. Fang, X. Zhang, Chin. J. Catal., 2017, 38, 240-252.

[285] W. Yu, J. Chen, T. Shang, L. Chen, L. Gu, T. Peng, Appl. Catal. B: Environ., 2017, 219, 693-704.

[286] Y. You, S. Wang, K. Xiao, T. Ma, Y. Zhang, H. Huang, ACS Sustain. Chem. Eng., 2018, 6, 16219-16227.

[287] J. Chen, Z. Shen, S. Lv, K. Shen, R. Wu, X.-F. Jiang, T. Fan, J. Chen, Y. Li, J. Mater. Chem. A, 2018, 6, 19631-19642.

[288] A. B. Yousaf, M. Imran, S. J. Zaidi, P. Kasak, Sci. Rep., 2017, 7, 6574.

[289] H. Katsumata, Y. Tachi, T. Suzuki, S. Kaneco, RSC Adv., 2014, 4, 21405-21409.

[290] Y. Z. Chen, C. Wang, Z. Y. Wu, Y. Xiong, Q. Xu, S. H. Yu, H. L. Jiang, Adv. Mater., 2015, 27, 5010-5016.

[291] L. Jiao, Y. Wang, H. L. Jiang, Q. Xu, Adv. Mater., 2018, 30, e1703663.

[292] J. D. Xiao, H. L. Jiang, Acc. Chem. Res., 2019, 52, 356-366.

[293] Y.-Z. Chen, R. Zhang, L. Jiao, H.-L. Jiang, Coord. Chem. Rev., 2018, 362, 1-23.

[294] S. J. Yang, S. Nam, T. Kim, J. H. Im, H. Jung, J. H. Kang, S. Wi, B. Park, C. R. Park, J. Am. Chem. Soc., 2013, 135, 7394-7397.

[295] X. Zhao, J. Feng, J. Liu, W. Shi, G. Yang, G. C. Wang, P. Cheng, Angew. Chem. Int. Ed., 2018, 57, 9790-9794.

[296] B. Zhu, R. Zou, Q. Xu, Adv. Energy Mater., 2018, 8, 1801193.

[297] H.-Q. Xu, S. Yang, X. Ma, J. Huang, H.-L. Jiang, ACS Catal., 2018, 8, 11615-11621.

[298] H. Liu, J. Zhang, D. Ao, Appl. Catal. B: Environ., 2018, 221, $433-442$. 
[299] C.-C. Wang, X.-H. Yi, P. Wang, Appl. Catal. B: Environ., 2019, 247, $24-48$.

[300] F. M. Zhang, J. L. Sheng, Z. D. Yang, X. J. Sun, H. L. Tang, M. Lu, H. Dong, F. C. Shen, J. Liu, Y. Q. Lan, Angew. Chem. Int. Ed., 2018, 57, 12106-12110.

[301] D. P. Kumar, H. Park, E. H. Kim, S. Hong, M. Gopannagari, D. A. Reddy, T. K. Kim, Appl. Catal. B: Environ., 2018, 224, 230-238.

[302] J. Chow, R. J. Kopp, P. R. Portney, Science, 2003, 302, 1528-1531.

[303] C. Du, L. Yang, F. Yang, G. Cheng, W. Luo, ACS Catal., 2017, 7, 4131-4137.

[304] J. Xu, Y. Qi, C. Wang, L. Wang, Appl. Catal. B: Environ., 2019, 241, 178-186.

[305] D. P. Kumar, J. Choi, S. Hong, D. A. Reddy, S. Lee, T. K. Kim, ACS Sustain. Chem. Eng., 2016, 4, 7158-7166.

[306] J. Yao, J. Chen, K. Shen, Y. Li, J. Mater. Chem. A, 2018, 6, 3571-3582.

[307] M. Lan, R.-M. Guo, Y. Dou, J. Zhou, A. Zhou, J.-R. Li, Nano Energy, 2017, 33, 238-246.
[308] J. He, Z. Yan, J. Wang, J. Xie, L. Jiang, Y. Shi, F. Yuan, F. Yu, Y. Sun, Chem. Commun., 2013, 49, 6761-6763.

[309] T. N. Nguyen, S. Kampouri, B. Valizadeh, W. Luo, D. Ongari, O. M. Planes, A. Zuttel, B. Smit, K. C. Stylianou, ACS Appl. Mater. Interfaces, 2018, 10, 30035-30039.

[310] B. Ma, P. Y. Guan, Q. Y. Li, M. Zhang, S. Q. Zang, ACS Appl. Mater. Interfaces, 2016, 8, 26794-26800.

[311] K. Jayaramulu, T. Toyao, V. Ranc, C. Rösler, M. Petr, R. Zboril, Y. Horiuchi, M. Matsuoka, R. A. Fischer, J. Mater. Chem. A, 2016, 4, 18037-18042.

[312] Z. Wang, Z. Jin, H. Yuan, G. Wang, B. Ma, J. Colloid Interface Sci,, 2018, 532, 287-299.

[313] S. Bala, I. Mondal, A. Goswami, U. Pal, R. Mondal, J. Mater. Chem. A, 2015, 3, 20288-20296.

[314] K. Pramoda, M. Kaur, U. Gupta, C. N. Rao, Dalton. Trans., 2016, 45, 13810-13816.

[315] Y. Zhang, Z. Jin, A. luan, G. Wang, Int. J. Hydrogen Energy, 2018, 43, 9914-9923.

\title{
光催化分解水制氢反应中助催化剂的作用及机理
}

肖 楠 ${ }^{\mathrm{a}, \mathrm{b}}$, 李松松 ${ }^{\mathrm{b}}$, 李旭力 ${ }^{\mathrm{b}}$, 戈 䂞, ${ }^{\mathrm{a}, \mathrm{b}^{*}}$, 高旸钦 ${ }^{\mathrm{b}}$, 李 宁 ${ }^{\mathrm{b}}$

a 中国石油大学(北京)重质油国家重点实验室, 新能源与材料学院, 北京102249

b 中国石油大学(北京)新能源与材料学院, 材料科学与工程系, 北京 1022449

\begin{abstract}
摘要: 近年来, 随着一次能源过度消耗所带来的能源和环境问题日益突出, 开发廉价、可持续的清洁能源备受关注. 光催化 分解水制氢可利用太阳能普遍率高和几乎免费等特点制取燃烧热值高、燃烧产物无污染的氢气能源. 自从1972年日本的 Fujishima教授和Honda教授首次发现 $\mathrm{TiO}_{2}$ 单晶电极光催化分解水可以产生氢气以来, 光催化制氢被认为是实现可持续制氢 最有潜力的方法之一. 有效地将太阳能转换为化学能的关键是设计高效的电荷分离和运输结构. 然而, 现有的大多数半导 体光催化剂因缺少活性位点、光生载流子易复合等缺点而无法达到较高的转换效率. 因此, 如何提高半导体光催化产氢的 转换效率是现阶段面对的重要问题. 在众多解决方法中, 助催化剂的引入可以为光催化制氢反应增加活性位点, 促进光生 载流子的有效分离, 进而有效地提高半导体光催化产氢速率. 本文总结了多种不同类型的助催化剂应用于光催化产氢研 究的最新进展, 详细讨论了助催化剂在增强光吸收、提供活性位点、增加催化剂稳定性和促进电荷分离等方面的作用, 阐 明了助催化剂在光催化分解水制氢中的反应机理, 同时还提出了光催化制氢的未来研究和预测.

本文将助催化剂分为以下几种类别进行讨论：(1)单一助催化剂,包括金属/合金、金属氧化物/氢氧化物、金属磷化物、 金属硫化物、碳基材料等助催化剂材料; (2)双助催化剂; (3)Z-Scheme助催化剂; (4)MOFs助催化剂. 近年来, 助催化剂材 料在光催化产氢中应用的发展趋势从当初价格昂贵的贵金属趋于价格相对低廉的非贵金属, 从单一体系趋于更复杂的体 系. 虽然现阶段关于助催化剂与基底之间的匹配还需要进一步研究, 但我们相信随着技术的发展, 这些问题都可以迎刃而 解. 希望在不久的将来, 可以精确设计和构建出具有高效光催化产氢活性的催化剂体系, 开发出更多新的可再生清洁能源, 从而缓解能源紧缺和环境恶化等棘手问题.
\end{abstract}

关键词: 助催化剂; 光催化; 析氢; 电荷分离; 分解水

收稿日期: 2019-09-26. 接受日期: 2019-10-17. 出版日期: 2020-04-05.

*通讯联系人. 电子信箱: gelei08@sina.com

基金来源：国家自然科学基金(51572295，21273285，21003157); 北京新星计划(2008B76); 中国石油大学(北京)科学基金 (KYJJ2012-06-20, 2462016YXBS05).

本文的电子版全文由Elsevier出版社在ScienceDirect上出版(http://www.sciencedirect.com/science/journal/18722067). 